\title{
Indirect Ortho Functionalization of Substituted Toluenes through Ortho Olefination of N,N-Dimethylbenzylamines Tuned by Acidity of Reaction Conditions
}

\author{
Guixin Cai, Ye Fu, Yizhou Li, Xiaobing Wan and Zhangjie Shi*
}

\begin{abstract}
Beijing National Laboratory of Molecular Sciences (BNLMS), PKU Green Chemistry Center and Key Laboratory of Bioorganic Chemistry and Molecular Engineering of Ministry of Education, College of Chemistry, Peking University, Beijing 100871 and Shanghai Key Laboratory of Green Chemistry and Chemical

Processes, East China Normal University, Shanghai 200062, China

E-mail: zshi@pku.edu.cn
\end{abstract}

\section{Table of Contents for SI:}

Analytical and spectral data for N,N-dimethylbenzylamines 1g-1r

SI $2-3$

analytical and spectral data for 3aa-3rd

SI $4-8$

Analytical and spectral data for toluene derivatives 4aa-4rd

SI 8-11

Mechanistic Investigation

SI 11-12

References

SI 12

NMR spectrum of compounds 


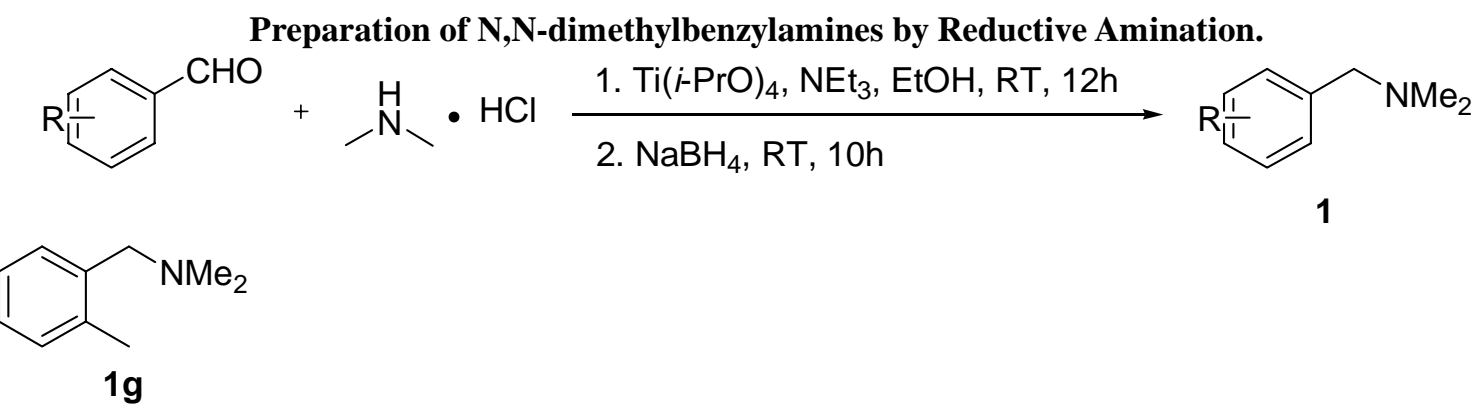

$\mathbf{N}, \mathbf{N}$-dimethyl(o-tolyl)methanamine (1g): $1 \mathrm{~g}$ was obtained as a light yellow oil (1.4 g, 65\% yield). ${ }^{1} \mathrm{H}$ NMR $\left(\mathrm{CDCl}_{3}, 300 \mathrm{MHz}\right) \delta 7.26(\mathrm{~m}, 1 \mathrm{H}), 7.22(\mathrm{~m}, 3 \mathrm{H}), 3.39(\mathrm{~s}, 2 \mathrm{H}), 2.39(\mathrm{~s}, 3 \mathrm{H}), 2.26(\mathrm{~s}, 6 \mathrm{H}) \mathrm{ppm} ;{ }^{13} \mathrm{C} \mathrm{NMR}\left(\mathrm{CDCl}_{3}\right.$, $75 \mathrm{MHz}) 137.2$, 137.0, 130.2, 129.8, 126.9, 125.5, 62.0, 45.5, 19.0 ppm; IR v 2942, 2855, 2762, 1459, 1021, $741 \mathrm{~cm}^{-1} \mathrm{MS}:(\mathrm{m} / \mathrm{z})(\%): 149(6)\left[\mathrm{M}^{+}\right], 42(100)$.<smiles>Cc1cccc(CN(C)C)c1</smiles>

$1 \mathrm{~h}$

$\mathbf{N}$, N, 3-trimethylbenzylamine(1h): $1 \mathbf{h}$ was obtained as red oil $\left(2.2 \mathrm{~g}, 63 \%\right.$ yield). ${ }^{1} \mathrm{H}$ NMR $\left(\mathrm{CDCl}_{3}, 300 \mathrm{MHz}\right)$ $\delta 7.08(\mathrm{~m}, 4 \mathrm{H}), 3.33(\mathrm{~s}, 2 \mathrm{H}), 2.28(\mathrm{~s}, 3 \mathrm{H}), 2.18(\mathrm{~s}, 6 \mathrm{H}) \mathrm{ppm} ;{ }^{13} \mathrm{C} \mathrm{NMR}\left(\mathrm{CDCl}_{3}, 75 \mathrm{MHz}\right) 138.7,137.7,129.7$, 128.0, 127.7, 126.0, 64.3, 45.3, 21.2 ppm; IR $v 2941,2814,2769,1456,1032,844,779,696 \mathrm{~cm}^{-1} \mathrm{MS}:(\mathrm{m} / \mathrm{z})$ (\%): $149(2)\left[\mathrm{M}^{+}\right], 58(100)$.<smiles>Cc1ccc(CN(C)C)cc1</smiles>

$1 \mathrm{i}$

$\mathbf{N}, \mathbf{N}, 4$-trimethylbenzylamine(1i): $1 \mathbf{i}$ was obtained as a light yellow oil (1.2 g, 52\% yield). ${ }^{1} \mathrm{H} \mathrm{NMR}\left(\mathrm{CDCl}_{3}\right.$, $300 \mathrm{MHz}) \delta 7.19(\mathrm{~d}, 2 \mathrm{H}, \mathrm{J}=7.2), 7.12(\mathrm{~d}, 2 \mathrm{H}, \mathrm{J}=7.5), 3.37(\mathrm{~s}, 2 \mathrm{H}), 2.32(\mathrm{~s}, 3 \mathrm{H}), 2.22(\mathrm{~s}, 6 \mathrm{H}) \mathrm{ppm} ;{ }^{13} \mathrm{C} \mathrm{NMR}$ $\left(\mathrm{CDCl}_{3}, 75 \mathrm{MHz}\right) 136.5,135.6,129.0,128.9,64.0,45.2,21.0$ ppm; IR $\vee 2943,2858,2766,1456,1031,855$, $800 \mathrm{~cm}^{-1}$ MS: (m/z) (\%): $149(5)\left[\mathrm{M}^{+}\right], 42(100)$.<smiles>COc1ccccc1CN(C)C</smiles>

$1 \mathrm{j}$

$\mathbf{N}$, N-dimethyl-2-methoxylbenzylamine(1j): $\mathbf{1 j}$ was obtained as a light yellow oil (1.3 g, 53\% yield). ${ }^{1} \mathrm{H}$ $\operatorname{NMR}\left(\mathrm{CDCl}_{3}, 300 \mathrm{MHz}\right) \delta 7.22(\mathrm{~m}, 2 \mathrm{H}), 6.87(\mathrm{~m}, 2 \mathrm{H}), 3.80(\mathrm{~s}, 3 \mathrm{H}), 3.43(\mathrm{~s}, 2 \mathrm{H}), 2.24(\mathrm{~s}, 6 \mathrm{H}) \mathrm{ppm} ;{ }^{13} \mathrm{C} \mathrm{NMR}$ $\left(\mathrm{CDCl}_{3}, 75 \mathrm{MHz}\right)$ 157.8, 130.7, 128.1, 126.7, 120.0, 110.3, 57.8, 55.3, 45.4 ppm; IR v 2960, 2858, 1713, 1255 , 1177,1020, $788 \mathrm{~cm}^{-1} \mathrm{MS}:(\mathrm{m} / \mathrm{z})(\%): 165(9)\left[\mathrm{M}^{+}\right], 58(100)$.<smiles>COc1ccc(CN(C)C)cc1</smiles>

$\mathbf{N}, \mathbf{N}$-dimethyl-4-methoxylbenzylamine(1k): $1 \mathbf{k}$ was obtained as a light yellow oil $\left(0.9 \mathrm{~g}, 35 \%\right.$ yield). ${ }^{1} \mathrm{H}$ $\operatorname{NMR}\left(\mathrm{CDCl}_{3}, 300 \mathrm{MHz}\right) \delta 7.18(\mathrm{~d}, 2 \mathrm{H}, \mathrm{J}=9), 6.82(\mathrm{~d}, 2 \mathrm{H}, \mathrm{J}=9), 3.76(\mathrm{~s}, 3 \mathrm{H}), 3.32(\mathrm{~s}, 2 \mathrm{H}), 2.18(\mathrm{~s}, 6 \mathrm{H}) \mathrm{ppm} ;{ }^{13} \mathrm{C}$ NMR $\left(\mathrm{CDCl}_{3}, 75 \mathrm{MHz}\right) 158.6,130.8,130.2,113.5,63.6,55.1,45.1 \mathrm{ppm}$; IR $\vee 2942,2813,2768,1511,1243$, 1028, $811 \mathrm{~cm}^{-1}$ MS: (m/z) (\%): $165(2)\left[\mathrm{M}^{+}\right], 58(100)$.<smiles>CN(C)Cc1cccc(F)c1</smiles>

11

$\mathbf{N}$, N-dimethyl-3-fluorobenzylamine(11): 11 was obtained as a light yellow oil (1.4 g, $61 \%$ yield). ${ }^{1} \mathrm{H}$ NMR $\left(\mathrm{CDCl}_{3}, 300 \mathrm{MHz}\right) \delta 7.24(\mathrm{~m}, 1 \mathrm{H}), 7.03(\mathrm{~m}, 2 \mathrm{H}), 6.88(\mathrm{~m}, 1 \mathrm{H}), 3.38(\mathrm{~s}, 2 \mathrm{H}), 2.21(\mathrm{~s}, 6 \mathrm{H}) \mathrm{ppm} ;{ }^{13} \mathrm{C} \mathrm{NMR}\left(\mathrm{CDCl}_{3}\right.$, $75 \mathrm{MHz}) 164.5,161.2,141.6,141.5,129.6,129.5,124.5,124.4,115.8,115.5,114.0,113.7,63.8,63.7,45.3$ 
ppm; IR v 2945, 2819, 2775, 1590, 1487, 1455, 1256, 783, $687 \mathrm{~cm}^{-1} \mathrm{MS}:(\mathrm{m} / \mathrm{z})(\%): 153(6)\left[\mathrm{M}^{+}\right], 58(100)$.<smiles>CN(C)Cc1ccc(F)cc1</smiles>

$\mathbf{N}$, N-dimethyl-4-fluorobenzylamine(1m): $1 \mathrm{~m}$ was obtained as a light yellow oil (1.5 g, $60 \%$ yield). ${ }^{1} \mathrm{H}$ NMR $\left(\mathrm{CDCl}_{3}, 300 \mathrm{MHz}\right) \delta 7.22(\mathrm{~m}, 2 \mathrm{H}), 6.95(\mathrm{~m}, 2 \mathrm{H}), 3.33(\mathrm{~s}, 2 \mathrm{H}), 2.19(\mathrm{~s}, 6 \mathrm{H}) \mathrm{ppm} ;{ }^{13} \mathrm{C} \mathrm{NMR}\left(\mathrm{CDCl}_{3}, 75 \mathrm{MHz}\right)$ $163.5,160.3,134.6,134.5,130.5,130.4,130.0,115.0,114.8,112.4,63.5,45.1$ ppm; IR $\vee 2925,2854,1614$, 1525, 1361, 1168, 803.6 $\mathrm{cm}^{-1}$ MS: (m/z) (\%): $153(51)\left[\mathrm{M}^{+}\right], 58(100)$.<smiles>CN(C)Cc1ccc(Cl)cc1</smiles>

$\mathbf{N}$, N-dimethyl-3-fluorobenzylamine(1n): 1n was obtained as a light yellow oil (1.8 g, $71 \%$ yield). ${ }^{1} \mathrm{H}$ NMR $\left(\mathrm{CDCl}_{3}, 300 \mathrm{MHz}\right) \delta 7.26(\mathrm{~m}, 4 \mathrm{H}), 3.37(\mathrm{~s}, 2 \mathrm{H}), 2.21(\mathrm{~s}, 6 \mathrm{H}) \mathrm{ppm} ;{ }^{13} \mathrm{C} \mathrm{NMR}\left(\mathrm{CDCl}_{3}, 75 \mathrm{MHz}\right) 137.4,132.7$, 130.3, 128.3, 63.5, 45.2 ppm; IR v 2944, 2817, 2769, 1490, 1086, 1015, 857, $801 \mathrm{~cm}^{-1} \mathrm{MS}:(\mathrm{m} / \mathrm{z})(\%): 169$ (1) $\left[\mathrm{M}^{+}\right], 58(100)$.<smiles>CN(C)Cc1ccc(C(F)(F)F)cc1</smiles>

$\mathbf{N}$, N-dimethyl-3-fluorobenzylamine(10): 10 was obtained as a light yellow oil (1.8 g, 58\% yield). ${ }^{1} \mathrm{H}$ NMR $\left(\mathrm{CDCl}_{3}, 300 \mathrm{MHz}\right) \delta 7.55$ (d, 2H, J=8.1), $7.40(\mathrm{~d}, 2 \mathrm{H}, \mathrm{J}=7.8), 3.44(\mathrm{~s}, 2 \mathrm{H}), 2.21(\mathrm{~s}, 6 \mathrm{H}) \mathrm{ppm} ;{ }^{13} \mathrm{C} \mathrm{NMR}\left(\mathrm{CDCl}_{3}\right.$, $75 \mathrm{MHz}) 142.9,129.4,125.14,125.09,125.04,124.99,63.7,45.3$ ppm; IR v 1329, 1171, 1129, 1069, 1020, 870, $817 \mathrm{~cm}^{-1}$ MS: (m/z) (\%): 203 (3) $\left[\mathrm{M}^{+}\right], 58(100)$.<smiles>CN(C)Cc1ccc2c(c1)OCO2</smiles>

$1 \mathrm{p}$

$\mathbf{N}$, N-dimethyl-piperonylamine(1p): 1p was obtained as light yellow oil $(2.0 \mathrm{~g}, 73 \%$ yield $) .{ }^{1} \mathrm{H} \mathrm{NMR}\left(\mathrm{CDCl}_{3}\right.$, $300 \mathrm{MHz}) \delta 6.79(\mathrm{~s}, 1 \mathrm{H}), 6.70(\mathrm{~m}, 2 \mathrm{H}), 5.89(\mathrm{~s}, 2 \mathrm{H}), 3.27(\mathrm{~s}, 2 \mathrm{H}), 2.17(\mathrm{~s}, 6 \mathrm{H}) \mathrm{ppm} ;{ }^{13} \mathrm{C} \mathrm{NMR}\left(\mathrm{CDCl}_{3}, 75 \mathrm{MHz}\right)$ 147.5, 146.5, 132.7, 122.0, 109.4, 107.7, 100.8, 64.0, 45.1 ppm; IR v 2943, 2815, 2768, 1499, 1442, 1240 , 1040, 930, $805 \mathrm{~cm}^{-1} \mathrm{MS}:(\mathrm{m} / \mathrm{z})(\%): 179(2)\left[\mathrm{M}^{+}\right], 58(100)$.<smiles>CN(C)Cc1ccc2ccccc2c1</smiles>

19

$\mathbf{N}$, N-dimethyl-2-naphthalenemethanamine (1q): 1q was obtained as a colorless oil ( $0.8 \mathrm{~g}, 30 \%$ yield). ${ }^{1} \mathrm{H}$ NMR $\left(\mathrm{CDCl}_{3}, 300 \mathrm{MHz}\right) \delta 7.20(\mathrm{~m}, 10 \mathrm{H}), 3.43(\mathrm{~s}, 2 \mathrm{H}), 2.09(\mathrm{~s}, 3 \mathrm{H}) \mathrm{ppm} ;{ }^{13} \mathrm{C} \mathrm{NMR}\left(\mathrm{CDCl}_{3}, 75 \mathrm{MHz}\right) 138.3$, 128.9, 128.2, 126.9, 61.8, 42.2 ppm; IR 3055,2941, 2854, 2768, 1455, 1366, 1261, 1031, 894, 814, $752 \mathrm{~cm}^{-1}$ MS: (m/z) (\%): $185(60)\left[\mathrm{M}^{+}\right], 141(100)$.<smiles>CCCCOc1ccccc1CN(C)C</smiles>

(2-isobutoxyphenyl)-N,N-dimethylmethanamine (1r): 1r was obtained as a colorless oil (3.5 g, $68 \%$ yield). ${ }^{1} \mathrm{H} \mathrm{NMR}\left(\mathrm{CDCl}_{3}, 300 \mathrm{MHz}\right) \delta 7.29(\mathrm{~m}, 1 \mathrm{H}), 7.20(\mathrm{~m}, 1 \mathrm{H}), 6.90(\mathrm{~m}, 1 \mathrm{H}), 6.82(\mathrm{~m}, 1 \mathrm{H}), 3.71(\mathrm{~d}, 2 \mathrm{H}, \mathrm{J}=6.3), 3.48$ (s, 2H), 2.27 (s, 6H), 2.09 (m, 1H), 1.04 (d, 6H, J=6.9) ppm; ${ }^{13} \mathrm{C} \mathrm{NMR}\left(\mathrm{CDCl}_{3}, 75 \mathrm{MHz}\right) 157.2,130.5,127.8$, 127.0, 119.9, 111.1, 74.2, 57.3, 45.4, 28.3, 19.3 ppm; IR v 2960, 2911, 2815, 2766, 1495, 1287, 1031, $753 \mathrm{~cm}^{-1}$ MS: (m/z) (\%): 233 (5) $\left[\mathrm{M}^{+}\right], 58$ (100), HRMS: Anal. Calcd. for $\mathrm{C}_{13} \mathrm{H}_{21} \mathrm{NO}$ 207.16231, Found: 207.16237.

Ortho-Functionalization of N,N-Dimethylbenzylamine with Different Alkenes. 


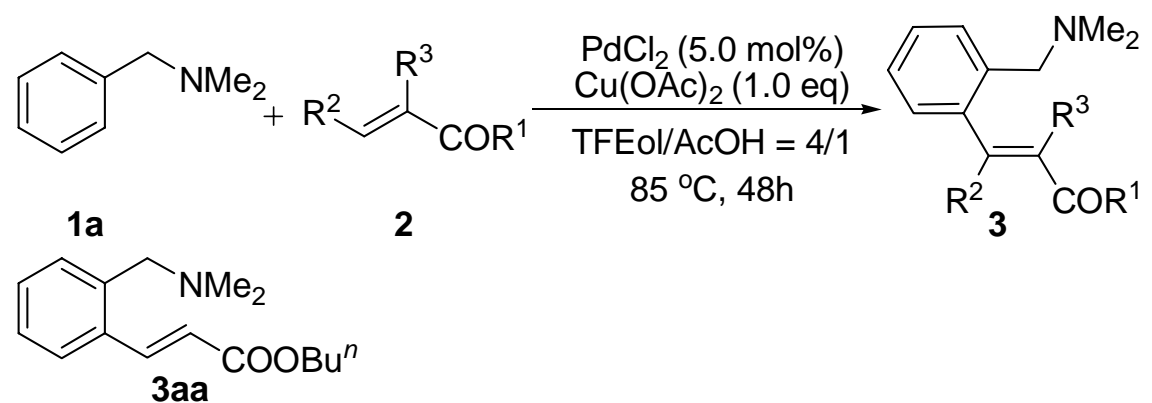

(E)-butyl 3-(2-((dimethylamino)methyl)phenyl)acrylate (3aa): Startinging from $1 \mathbf{a}(75 \mu \mathrm{L}, 0.5 \mathrm{mmol})$ with 2a $(145 \mu \mathrm{L}, 1.0 \mathrm{mmol})$ to afford 3aa $(112 \mathrm{mg})$, yield $86 \% .{ }^{1} \mathrm{H} \mathrm{NMR}\left(\mathrm{CDCl}_{3}, 300 \mathrm{MHz}\right) \delta 8.21(\mathrm{~d}, 1 \mathrm{H}, \mathrm{J}=15.9)$, $7.61(\mathrm{~d}, 1 \mathrm{H}, \mathrm{J}=6.3), 7.33-7.27(\mathrm{~m}, 3 \mathrm{H}), 6.39$ (d, 1H, J=15.9), 4.23 (t, 2H, J=6.6), 3.51 (s, 2H), $2.26(\mathrm{~s}, 6 \mathrm{H}), 1.71$ $(\mathrm{m}, 2 \mathrm{H}), 1.46(\mathrm{~m}, 2 \mathrm{H}), 0.98(\mathrm{t}, 3 \mathrm{H}, \mathrm{J}=7.2) \mathrm{ppm} ;{ }^{13} \mathrm{C} \mathrm{NMR}\left(\mathrm{CDCl}_{3}, 75 \mathrm{MHz}\right) 167.3,142.3,138.3,134.4,130.9$, 129.7, 127.7, 126.6, 119.3, 64.3, 61.7, 45.2, 30.7, 19.1, 13.7 ppm; IR v 2959, 1713, 1308, 1170, $766 \mathrm{~cm}^{-1}$; MS: $(\mathrm{m} / \mathrm{z})(\%): 261(8)\left[\mathrm{M}^{+}\right], 115$ (100), HRMS: Anal. Calcd. for $\mathrm{C}_{16} \mathrm{H}_{23} \mathrm{NO}_{2}$ 261.17310, Found: 261.17288.<smiles>CCOC(=O)C=Cc1ccccc1CN(C)C</smiles>

(E)-ethyl 3-(2-((dimethylamino)methyl)phenyl)acrylate (3ab): Startinging from 1a (75 $\mu \mathrm{L}, 0.5 \mathrm{mmol})$ with 2b $(108 \mu \mathrm{L}, 1.0 \mathrm{mmol})$ to afford 3ab $(99 \mathrm{mg})$, yield 85\%. ${ }^{1} \mathrm{H}$ NMR $\left(\mathrm{CDCl}_{3}, 300 \mathrm{MHz}\right) \delta 8.09(\mathrm{~d}, 1 \mathrm{H}, \mathrm{J}=15.6)$, $7.50(\mathrm{~d}, 1 \mathrm{H}, \mathrm{J}=6.3), 7.22-7.18(\mathrm{~m}, 3 \mathrm{H}), 6.27(\mathrm{~d}, 1 \mathrm{H}, \mathrm{J}=15.0), 4.16(\mathrm{q}, 2 \mathrm{H}, \mathrm{J}=6.6), 3.40(\mathrm{~s}, 2 \mathrm{H}), 2.15(\mathrm{~s}$, $6 \mathrm{H}), 1.24$ (t, 3H,) ppm; ${ }^{13} \mathrm{C} \mathrm{NMR}\left(\mathrm{CDCl}_{3}, 75 \mathrm{MHz}\right) 167.0,142.5,138.1,134.2,130.7,129.5,127.6,126.4$, 119.1, 61.6, 60.3, 45.1, 14.22 ppm; IR v 2978, 2768, 1712, 1174, $766 \mathrm{~cm}^{-1} ; \mathrm{MS}:(\mathrm{m} / \mathrm{z})(\%): 233(10)\left[\mathrm{M}^{+}\right], 43$ (100), HRMS: Anal. Calcd. for $\mathrm{C}_{14} \mathrm{H}_{19} \mathrm{NO}_{2} 233.14158$, Found: 233.14123.<smiles>COC(=O)C=Cc1ccccc1CN(C)C</smiles>

(E)-3-(2-((dimethylamino)methyl)phenyl)acrylamide (3ac): Starting from 1a (75 $\mu \mathrm{L}, 0.5 \mathrm{mmol})$ with $\mathbf{2 c}$ (71 mg, $1.0 \mathrm{mmol})$ to afford 3ac $(84 \mathrm{mg})$, yield 66\%. ${ }^{1} \mathrm{H} \mathrm{NMR}\left(\mathrm{CDCl}_{3}, 300 \mathrm{MHz}\right) \delta 8.03(\mathrm{~d}, 1 \mathrm{H}, \mathrm{J}=15.6), 7.53$ (d, 1H, J=7.2), 7.29-7.24 (m, 3H), 6.39 (d, 1H, J=17.4), $5.9(\mathrm{br}, 2 \mathrm{H}), 3.50(\mathrm{~s}, 2 \mathrm{H}), 2.22(\mathrm{~s}, 6 \mathrm{H}) \mathrm{ppm} ;{ }^{13} \mathrm{C} \mathrm{NMR}$ $\left(\mathrm{CDCl}_{3}, 75 \mathrm{MHz}\right) 168.1,139.6,137.8,134.4,130.8,129.3,127.6,126.4,121.2,61.5,45.1$ ppm; IR $v$ 3441, 3173, 2819, 1669, 1601, 1396, $741 \mathrm{~cm}^{-1}$; MS: (m/z) (\%): 204 (10) [M+], 116 (100), HRMS: Anal. Calcd. for $\mathrm{C}_{12} \mathrm{H}_{16} \mathrm{~N}_{2} \mathrm{O}$ 204.12626, Found: 204.12617.

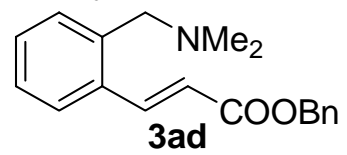

(E)-benzyl 3-(2-((dimethylamino)methyl)phenyl)acrylate (3ad): Starting from $1 \mathrm{a}(75 \mu \mathrm{L}, 0.5 \mathrm{mmol})$ with 2d $(162 \mathrm{mg}, 1.0 \mathrm{mmol})$ to afford 3ad $(127 \mathrm{mg})$, yield $86 \% .{ }^{1} \mathrm{H} \mathrm{NMR}\left(\mathrm{CDCl}_{3}, 300 \mathrm{MHz}\right) \delta 8.26(\mathrm{~d}, 1 \mathrm{H}, \mathrm{J}=15.9)$, $7.59(\mathrm{~d}, 1 \mathrm{H}, \mathrm{J}=8.1), 7.44-7.24(\mathrm{~m}, 9 \mathrm{H}), 6.42(\mathrm{~d}, 1 \mathrm{H}, \mathrm{J}=15.9), 5.25(\mathrm{~s}, 2 \mathrm{H}), 3.48(\mathrm{~s}, 2 \mathrm{H}), 2.19(\mathrm{~s}, 6 \mathrm{H}) \mathrm{ppm} ;{ }^{13} \mathrm{C}$ $\mathrm{NMR}\left(\mathrm{CDCl}_{3}, 75 \mathrm{MHz}\right) 166.7,142.9,138.2,136.0,134.0,130.7,129.6,128.4,128.1,127.6,126.5,118.6,66.2$, 61.7, 45.2 ppm; IR 2817, 1713, 1456, 1308, 1164, $766 \mathrm{~cm}^{-1}$; MS: (m/z) (\%): 295 (10) [M+1, 204 (100), HRMS: Anal. Calcd. for $\mathrm{C}_{19} \mathrm{H}_{22} \mathrm{NO}_{2}$ 295.15723. Found: 295.15774 .<smiles>CN(C)Cc1ccccc1C=CC(N)=O</smiles>

(E)-3-(2-((dimethylamino)methyl)phenyl)acrylamide (3ae): Start from 1a (75 $\mu \mathrm{L}, 0.5$ mmol) with 2e (71 $\mathrm{mg}, 1.0 \mathrm{mmol})$ to afford 3ae $(84 \mathrm{mg})$, yield $82 \% .{ }^{1} \mathrm{H} \mathrm{NMR}\left(\mathrm{CDCl}_{3}, 300 \mathrm{MHz}\right) \delta 8.03(\mathrm{~d}, 1 \mathrm{H}, \mathrm{J}=15.6), 7.53(\mathrm{~d}$, $1 \mathrm{H}, \mathrm{J}=7.2), 7.29-7.24(\mathrm{~m}, 3 \mathrm{H}), 6.39(\mathrm{~d}, 1 \mathrm{H}, \mathrm{J}=17.4), 5.9(\mathrm{br}, 2 \mathrm{H}), 3.50(\mathrm{~s}, 2 \mathrm{H}), 2.22(\mathrm{~s}, 6 \mathrm{H}) \mathrm{ppm} ;{ }^{13} \mathrm{C} \mathrm{NMR}$ $\left(\mathrm{CDCl}_{3}, 75 \mathrm{MHz}\right) 168.1,139.6,137.8,134.4,130.8,129.3,127.6,126.4,121.2,61.5,45.1$ ppm; IR 3441, 3173,

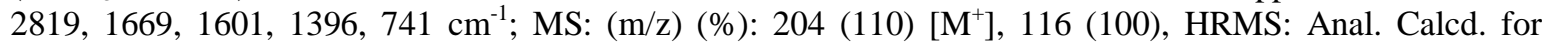
$\mathrm{C}_{12} \mathrm{H}_{16} \mathrm{~N}_{2} \mathrm{O}$ 204.12626, Found: 204.12617. 


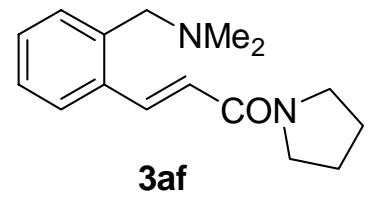

(E)-3-(2-((dimethylamino)methyl)phenyl)-1-(pyrrolidin-1-yl)prop-2-en-1-one (3af): Starting from 1a (75 $\mu \mathrm{L}, 0.5 \mathrm{mmol})$ with $3 \mathbf{f}(125 \mathrm{mg}, 1.0 \mathrm{mmol})$ to afford 3af $(71 \mathrm{mg})$, yield $54 \% .{ }^{1} \mathrm{H} \mathrm{NMR}\left(\mathrm{CDCl}_{3}, 300 \mathrm{MHz}\right) \delta$ $8.13(\mathrm{~d}, 1 \mathrm{H}, \mathrm{J}=15.6), 7.57$ (d, 1H, J=6.6), 7.33-7.26 (m, 3H), 6.68 (d, 1H, J=15.6), $3.61(\mathrm{~m}, 4 \mathrm{H}), 3.52(\mathrm{~s}, 2 \mathrm{H})$, $2.25(\mathrm{~s}, 6 \mathrm{H}), 2.03-1.88(\mathrm{~m}, 4 \mathrm{H}) \mathrm{ppm} ;{ }^{13} \mathrm{C} \mathrm{NMR}\left(\mathrm{CDCl}_{3}, 75 \mathrm{MHz}\right) 164.7,139.3,138.1,135.0,128.8,127.2$, 126.4, 120.2, 61.4, 45.4, 45.9, 45.3, 26.0, 24.2 ppm; IR v 2972, 2872, 1652, 1609, 1426, 1167, $1014,768 \mathrm{~cm}^{-1}$; MS: (m/z) (\%): $258(30)\left[\mathrm{M}^{+}\right], 70$ (100), HRMS: Anal. Calcd. for $\mathrm{C}_{16} \mathrm{H}_{22} \mathrm{~N}_{2} \mathrm{O}$ 258.17321, Found: 258.17318.<smiles>C=C(Cc1ccccc1CN(C)C)C(=O)OCC</smiles>

Ethyl 2-(2-((dimethylamino)methyl)benzyl)acrylate (3ag): Starting from $\mathbf{1 a}(75 \mu \mathrm{L}, 0.5$ mmol) with $\mathbf{2 g}$ $(124 \mu \mathrm{L}, 1.0 \mathrm{mmol})$ to afford $3 \mathbf{a g}(66 \mathrm{mg})$, yield 53\%. ${ }^{1} \mathrm{H} \mathrm{NMR}\left(\mathrm{CDCl}_{3}, 300 \mathrm{MHz}\right) \delta 7.27-7.10(\mathrm{~m}, 4 \mathrm{H}), 6.17(\mathrm{q}$, $1 \mathrm{H}, \mathrm{J}=1.2), 5.16(\mathrm{t}, 1 \mathrm{H}), 4.19(\mathrm{q}, 2 \mathrm{H}, \mathrm{J}=6.9), 3.73(\mathrm{~s}, 2 \mathrm{H}), 3.32(\mathrm{~s} 2 \mathrm{H}) 2.18(\mathrm{~s}, 6 \mathrm{H}, 2.4), 1.26(\mathrm{t}, 3 \mathrm{H}) \mathrm{ppm} ;{ }^{13} \mathrm{C}$ NMR $\left(\mathrm{CDCl}_{3}, 75 \mathrm{MHz}\right)$ 167.0, 140.1, 137.6, 137.3, 130.2, 130.1, 127.1, 126.3, 125.4, 61.6, 60.6, 45.4, 34.2,

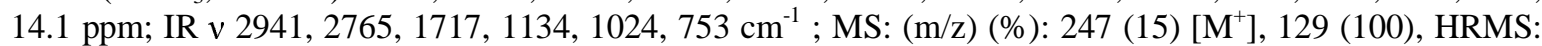
Anal. Calcd. for $\mathrm{C}_{15} \mathrm{H}_{21} \mathrm{NO}_{2} 247.15709$, Found: 247.15723 .

Ortho Olefination of Different Substituted Benzyl Amines.
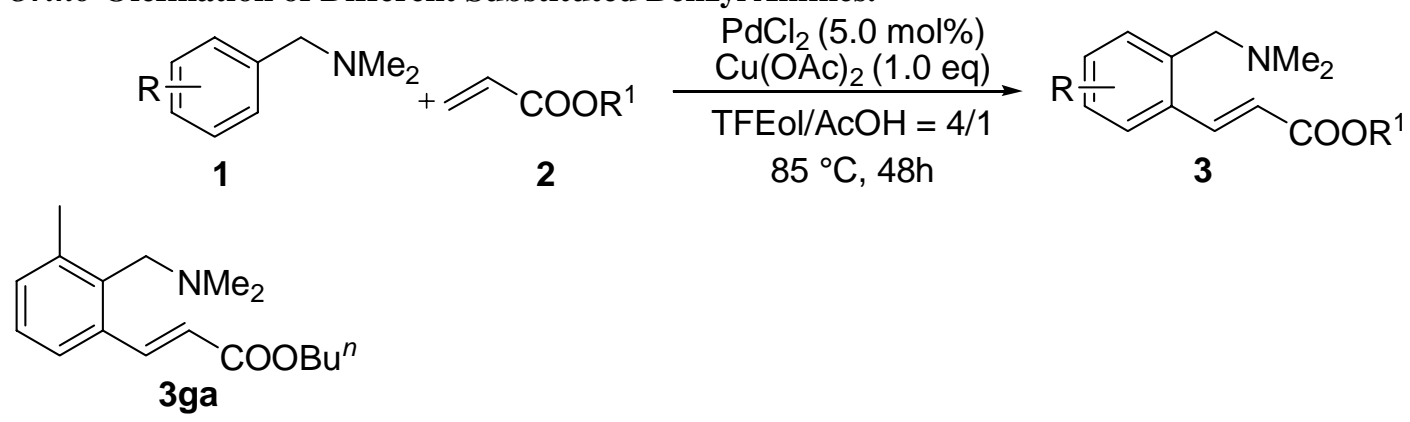

(E)-butyl 3-(2-((dimethylamino)methyl)-3-methylphenyl)acrylate (3ga): Start from $1 \mathrm{~g}$ (75 $\mathrm{mg}, 0.5 \mathrm{mmol})$ with $2 \mathbf{a}(145 \mu \mathrm{L}, 1.0 \mathrm{mmol})$ to afford $3 \mathrm{ga}(103 \mathrm{mg})$, yield $75 \% .{ }^{1} \mathrm{H} \mathrm{NMR}\left(\mathrm{CDCl}_{3}, 300 \mathrm{MHz}\right) \delta 8.25(\mathrm{~d}, 1 \mathrm{H}$, $\mathrm{J}=15.9), 7.41(\mathrm{~m}, 1 \mathrm{H}), 7.15(\mathrm{~m}, 2 \mathrm{H}), 6.29(\mathrm{~d}, 1 \mathrm{H}, \mathrm{J}=15.9), 4.18(\mathrm{t}, 2 \mathrm{H}, \mathrm{J}=6.6), 3.47(\mathrm{~s}, 2 \mathrm{H}), 2.37(\mathrm{~s}, 3 \mathrm{H}), 2.21(\mathrm{~s}$, $6 \mathrm{H}), 1.66(\mathrm{~m}, 2 \mathrm{H}), 1.41(\mathrm{~m}, 2 \mathrm{H}), 0.93(\mathrm{t}, 3 \mathrm{H}, \mathrm{J}=7.8) \mathrm{ppm} ;{ }^{13} \mathrm{C} \mathrm{NMR}\left(\mathrm{CDCl}_{3}, 75 \mathrm{MHz}\right) 167.2,143.6,138.3$, 136.5, 135.1, 131.9, 127.2, 124.4, 119.0, 64.2, 56.3, 45.0, 30.7, 20.0, 19.2, 13.7 ppm; IR 2960, 1714, 1311, 1164, $788 \mathrm{~cm}^{-1}$; MS: (m/z) (\%): 275 (33) [M+], 129 (100), HRMS: Anal. Calcd. for $\mathrm{C}_{17} \mathrm{H}_{25} \mathrm{NO}_{2}$ 275.18853, Found: 275.18934.

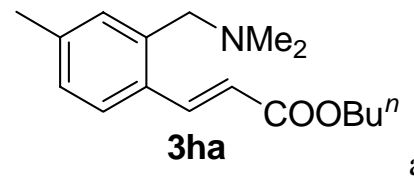<smiles>CCCCCCCCOC(=O)N(C)Cc1cccc(C)c1/C=C/c1ccccc1</smiles>

(E)-butyl 3-(2-((dimethylamino)methyl)-3-methylphenyl)acrylate $\quad$ (3ha) and (E)-butyl 3-(2-((dimethylamino)methyl)-6-methylphenyl)acrylate (3ha'): Start from $\mathbf{1 h}$ (75 $\mathrm{mg}, 0.5 \mathrm{mmol})$ with 2a (145 $\mathrm{LL}, 1.0 \mathrm{mmol})$ to afford a mixture of 3ha and 3ha' $\left(104 \mathrm{mg}\right.$, 3ha:3ha'=9:1), yield 75\%. ${ }^{1} \mathrm{H} \mathrm{NMR}\left(\mathrm{CDCl}_{3}\right.$, $300 \mathrm{MHz}) \delta 8.12(\mathrm{~d}, 0.69 \mathrm{H}, \mathrm{J}=16.2), 7.95(\mathrm{~d}, 0.04 \mathrm{H}, \mathrm{J}=16.2), 7.47(\mathrm{~d}, 0.76 \mathrm{H}, \mathrm{J}=7.8) 7.06(\mathrm{~m}, 2.06 \mathrm{H}), 6.30(\mathrm{~d}$, $0.76 \mathrm{H}, \mathrm{J}=15.9), 6.18(\mathrm{~d}, 0.10 \mathrm{H}, \mathrm{J}=16.5), 4.16(\mathrm{t}, 2.00 \mathrm{H}), 3.41(\mathrm{~s}, 1.77 \mathrm{H}), 3.32(\mathrm{~s}, 0.25 \mathrm{H}), 2.30(\mathrm{~s}, 3.16 \mathrm{H}), 2.20$ $(\mathrm{s}, 6.73 \mathrm{H}), 1.65(\mathrm{~m}, 2.21 \mathrm{H}), 1.40(\mathrm{~m}, 2.13 \mathrm{H}), 0.92(\mathrm{t}, 3.12 \mathrm{H}) \mathrm{ppm} ;{ }^{13} \mathrm{C} \mathrm{NMR}\left(\mathrm{CDCl}_{3}, 75 \mathrm{MHz}\right) 167.2,143.0$, $142.0,139.7,138.1,137.4,131.3,131.2,129.4,128.2,128.0,127.8,126.3,124.0,117.9,64.0,62.1,61.6,45.2$, 45.0, 30.6, 21.0, 19.1, 13.6 ppm; IR v2816, 1713, 1633, 1464, 1311, 1261, 1173, 1029, $842 \mathrm{~cm}^{-1} \mathrm{MS}:(\mathrm{m} / \mathrm{z})$ (\%): 275 (28) $\left[\mathrm{M}^{+}\right], 58$ (100), HRMS: Anal. Calcd. for $\mathrm{C}_{17} \mathrm{H}_{25} \mathrm{NO}_{2}$ 275.18853, Found: 275.18849. 


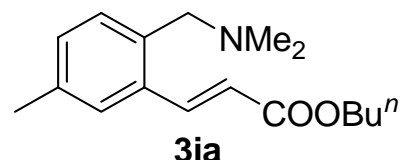

(E)-butyl 3-(2-((dimethylamino)methyl)-5-methylphenyl)acrylate (3ia): Start from 1i (75 mg, $0.5 \mathrm{mmol})$ with $2 \mathbf{a}(145 \mu \mathrm{L}, 1.0 \mathrm{mmol})$ to afford 3ia $(97 \mathrm{mg})$, yield $70 \%$. ${ }^{1} \mathrm{H} \mathrm{NMR}\left(\mathrm{CDCl}_{3}, 300 \mathrm{MHz}\right) \delta 8.14(\mathrm{~d}, 1 \mathrm{H}$, $\mathrm{J}=15.9), 7.39(\mathrm{~s}, 1 \mathrm{H}), 7.11(\mathrm{~m}, 2 \mathrm{H}), 6.34(\mathrm{~d}, 1 \mathrm{H}, \mathrm{J}=15.9), 4.17(\mathrm{t}, 2 \mathrm{H}), 3.41(\mathrm{~s}, 2 \mathrm{H}), 2.29(\mathrm{~s}, 3 \mathrm{H}), 2.19(\mathrm{~s}, 6 \mathrm{H})$, $1.64(\mathrm{~m}, 2 \mathrm{H}), 1.42(\mathrm{~m}, 2 \mathrm{H}), 0.93(\mathrm{t}, 3 \mathrm{H}, \mathrm{J}=7.2) \mathrm{ppm} ;{ }^{13} \mathrm{C} \mathrm{NMR}\left(\mathrm{CDCl}_{3}, 75 \mathrm{MHz}\right) 170.9,146.1,140.8,139.1$, 137.7, 134.5, 134.1, 132.8, 132.6, 130.8, 122.5, 67.9, 65.2, 48.9, 34.5, 24.7, 22.9, 17.4 ppm; IR v 2959, 2816,

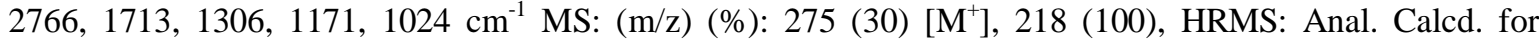
$\mathrm{C}_{17} \mathrm{H}_{25} \mathrm{NO}_{2} 275.18853$, Found: 275.18823.<smiles>COC(=O)/C=C/c1cccc(OC)c1CN</smiles>

(E)-butyl 3-(2-((dimethylamino)methyl)-3-methoxyphenyl)acrylate (3ja): Start from 1j (82 mg, $0.5 \mathrm{mmol})$ with $2 \mathbf{a}(145 \mu \mathrm{L}, 1.0 \mathrm{mmol})$ to afford $3 \mathbf{j a}(110 \mathrm{mg})$, yield $75 \% .{ }^{1} \mathrm{H} \mathrm{NMR}\left(\mathrm{CDCl}_{3}, 300 \mathrm{MHz}\right) \delta 8.16(\mathrm{~d}, 1 \mathrm{H}$, $\mathrm{J}=15.9), 7.21(\mathrm{~m}, 2 \mathrm{H}), 6.87(\mathrm{~m}, 1 \mathrm{H}), 6.33$ (d, 1H, J=15.9), 4.17 (t, 2H, J=6.6), 3.80 (s, 3H), 3.53 (s, $2 \mathrm{H}), 2.22(\mathrm{~s}$, $6 \mathrm{H}), 1.67$ (m, 2H), $1.42(\mathrm{~m}, 2 \mathrm{H}), 0.93$ (t, 3H, J=7.5) ppm; ${ }^{13} \mathrm{C} \mathrm{NMR}\left(\mathrm{CDCl}_{3}, 75 \mathrm{MHz}\right) 167.1,158.4,142.7$ 136.1 128.3, 126.6, 119.7, 118.7, 111.8, 64.3, 55.7, 52.4, 45.0, 30.7, 19.2, 13.7 ppm; IR v 2959, 2815, 2766, 1713, 1171, $1022 \mathrm{~cm}^{-1} \mathrm{MS}:(\mathrm{m} / \mathrm{z})(\%):$ 291(45) [ $\left.\mathrm{M}^{+}\right], 234$ (100), HRMS: Anal. Calcd. for $\mathrm{C}_{17} \mathrm{H}_{25} \mathrm{NO}_{3}$ 291.18344, Found: 291.18360.<smiles>COC(=O)C=Cc1cc(OC)ccc1CN(C)C</smiles>

(E)-butyl 3-(2-((dimethylamino)methyl)-5-methoxyphenyl)acrylate (3ka): Start from 1k (82 mg, $0.5 \mathrm{mmol})$ with $2 \mathbf{a}(145 \mu \mathrm{L}, 1.0 \mathrm{mmol})$ to afford 3ka $(111 \mathrm{mg})$, yield $76 \% .{ }^{1} \mathrm{H} \mathrm{NMR}\left(\mathrm{CDCl}_{3}, 300 \mathrm{MHz}\right) \delta 8.15(\mathrm{~d}, 1 \mathrm{H}$, $\mathrm{J}=15.9), 7.19(\mathrm{~d}, 1 \mathrm{H}, \mathrm{J}=8.4), 7.12(\mathrm{~d}, 1 \mathrm{H}, 2.1), 6.88(\mathrm{~m}, 1 \mathrm{H}), 6.37$ (d, 1H, J=16.2), 4.21 (t, 2H, J=6.6), 3.85 (s, $3 \mathrm{H}), 3.43(\mathrm{~s}, 2 \mathrm{H}), 2.22(\mathrm{~s}, 6 \mathrm{H}), 1.70(\mathrm{~m}, 2 \mathrm{H}), 1.47(\mathrm{~m}, 2 \mathrm{H}), 0.97(\mathrm{t}, 3 \mathrm{H}, \mathrm{J}=6.9) \mathrm{ppm} ;{ }^{13} \mathrm{C} \mathrm{NMR}\left(\mathrm{CDCl}_{3}, 75 \mathrm{MHz}\right)$ 167.0, 158.8, 142.2, 135.2, 132.0, 130.7, 119.1, 115.3, 111.1, 64.2, 61.1, 55.1, 45.0, 30.6, 19.1, 13.6 ppm; IR v 2960, 2815, 1713, 1173, 1022, 852, $805 \mathrm{~cm}^{-1} \mathrm{MS}:(\mathrm{m} / \mathrm{z})(\%): 291$ (40) [M+1, 234 (100), HRMS: Anal. Calcd. for $\mathrm{C}_{17} \mathrm{H}_{25} \mathrm{NO}_{3} 291.18344$, Found: 291.18281.<smiles>CCOC(=O)/C=C/c1ccc(F)cc1CN(C)C</smiles><smiles>COC(=O)/C=C/c1c(F)cccc1CN(C)C</smiles>

(E)-butyl 3-(2-((dimethylamino)methyl)-4-fluorophenyl)acrylate (3la) and (E)-butyl 3-(2-((dimethylamino)methyl)-6-fluorophenyl)acrylate (3la'): Start from 11 (76 mg, $0.5 \mathrm{mmol})$ with 2a $(145 \mu \mathrm{L}, 1.0 \mathrm{mmol})$ to afford a mixture of 3la and 3la' $\left(122 \mathrm{mg}\right.$, 3la:3la'=5:3), yield 86\%. ${ }^{1} \mathrm{H} \mathrm{NMR}\left(\mathrm{CDCl}_{3}\right.$, $300 \mathrm{MHz}) \delta 8.11(\mathrm{~d}, 0.69 \mathrm{H}, \mathrm{J}=16.2), 7.95(\mathrm{~d}, 0.04 \mathrm{H}, \mathrm{J}=16.2), 7.46(\mathrm{~d}, 0.76 \mathrm{H}, \mathrm{J}=8.1), 7.05(\mathrm{~m}, 2.06 \mathrm{H}), 6.30(\mathrm{~d}$, $0.76 \mathrm{H}, \mathrm{J}=15.6), 6.18(\mathrm{~d}, 0.10 \mathrm{H}, \mathrm{J}=16.2), 4.16(\mathrm{t}, 2.00 \mathrm{H}, \mathrm{J}=6.9), 4.41(\mathrm{~s}, 1.77 \mathrm{H}), 4.32(\mathrm{~s}, 0.25 \mathrm{H}), 2.31(\mathrm{~s}, 3.16 \mathrm{H})$, $2.20(\mathrm{~s}, 6.73 \mathrm{H}), 1.64(\mathrm{~m}, 2.21 \mathrm{H}), 1.39(\mathrm{~m}, 2.13 \mathrm{H}), 0.92(\mathrm{t}, 3.12 \mathrm{H}) \mathrm{ppm} ;{ }^{13} \mathrm{C} \mathrm{NMR}\left(\mathrm{CDCl}_{3}, 75 \mathrm{MHz}\right) 167.3$, $163.6,161.7, \quad 160.3,141.0,140.9,136.0,130.1,129.9,129.7,128.4,128.3,126.3,126.2,123.8,123.6,122.3$, $122.2,118.8,117.2,116.9,115.3,115.0,114.6,114.3,64.2,61.8,61.3,45.2,45.1,30.6,19.1,13.6$ ppm; IR v 2960, 2866, 2819, 2770, 1715, 1313, 1252, ,1173, 1029, $844 \mathrm{~cm}^{-1} \mathrm{MS}:(\mathrm{m} / \mathrm{z})(\%): 279(40)\left[\mathrm{M}^{+}\right], 178(100)$, HRMS: Anal. Calcd. for $\mathrm{C}_{16} \mathrm{H}_{22} \mathrm{NO}_{2} \mathrm{~F} 279.16346$, Found: 279.16296.<smiles>CCOC(=O)/C=C/c1cc(F)ccc1CN(C)C</smiles> 
(E)-butyl 3-(2-((dimethylamino)methyl)-5-fluorophenyl)acrylate (3ma): Start from 1m (76 mg, $0.5 \mathrm{mmol})$ with $2 \mathrm{a}(145 \mu \mathrm{L}, 1.0 \mathrm{mmol})$ to afford $3 \mathrm{ma}(106 \mathrm{mg})$, yield $76 \% .{ }^{1} \mathrm{H} \mathrm{NMR}\left(\mathrm{CDCl}_{3}, 300 \mathrm{MHz}\right) \delta 8.13(\mathrm{~d}, 1 \mathrm{H}$, $\mathrm{J}=16.2), 7.28(\mathrm{~m}, 2 \mathrm{H}), 7.00(\mathrm{~m}, 1 \mathrm{H}), 6.34(\mathrm{~d}, 1 \mathrm{H}, \mathrm{J}=16.2), 4.21(\mathrm{t}, 2 \mathrm{H}, \mathrm{J}=6.9), 3.45(\mathrm{~s}, 2 \mathrm{H}), 2.22(\mathrm{~s}, 6 \mathrm{H}), 1.67$ $(\mathrm{m}, 2 \mathrm{H}), 1.43(\mathrm{~m}, 2 \mathrm{H}), 0.96(\mathrm{t}, 3 \mathrm{H}, \mathrm{J}=7.2) \mathrm{ppm} ;{ }^{13} \mathrm{C} \mathrm{NMR}\left(\mathrm{CDCl}_{3}, 75 \mathrm{MHz}\right) 166.7,163.7,160.4,141.1,141.1$, 136.2, 136.1, 134.12, 134.08, 132.4, 132.3, 120.2, 116.4, 116.1, 113.1, 112.8, 64.4, 61.1, 45.1, 30.7, 19.1, 13.7

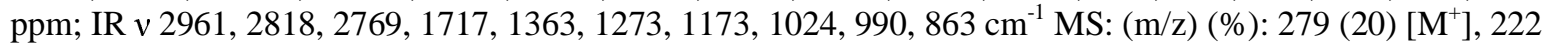
(100), HRMS: Anal. Calcd. for $\mathrm{C}_{16} \mathrm{H}_{22} \mathrm{NO}_{2} \mathrm{~F} 279.16346$, Found: 279.16329.

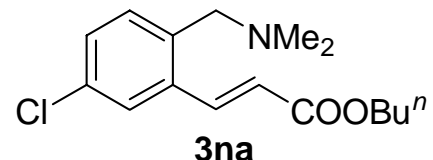

(E)-butyl 3-(5-chloro-2-((dimethylamino)methyl)phenyl)acrylate (3na): Start from 1n (85 mg, $0.5 \mathrm{mmol})$ with 2a $(145 \mu \mathrm{L}, 1.0 \mathrm{mmol})$ to afford 3na $(127 \mathrm{mg})$, yield $85 \% .{ }^{1} \mathrm{H} \mathrm{NMR}\left(\mathrm{CDCl}_{3}, 300 \mathrm{MHz}\right) \delta 8.06(\mathrm{~d}, 1 \mathrm{H}$, $\mathrm{J}=15.9), 7.52(\mathrm{~s}, 1 \mathrm{H}), 7.20(\mathrm{~m}, 2 \mathrm{H}), 6.33(\mathrm{~d}, 1 \mathrm{H}, \mathrm{J}=15.9), 4.17(\mathrm{t}, 2 \mathrm{H}, \mathrm{J}=6.6), 3.40(\mathrm{~s}, 2 \mathrm{H}), 2.18(\mathrm{~s}, 6 \mathrm{H}), 1.64(\mathrm{~m}$, $2 \mathrm{H}), 1.41(\mathrm{~m}, 2 \mathrm{H}), 0.92(\mathrm{t}, 3 \mathrm{H}, \mathrm{J}=7.8) \mathrm{ppm} ;{ }^{13} \mathrm{C} \mathrm{NMR}\left(\mathrm{CDCl}_{3}, 75 \mathrm{MHz}\right) 166.7,140.8,136.7,135.9,133.4$, 131.9, 129.2, 26.3, 120.3, 64.4, 61.1, 45.1, 30.6, 19.1, 13.7 ppm; IR v2960, 2866, 2818, 2769, 1715, 1637,

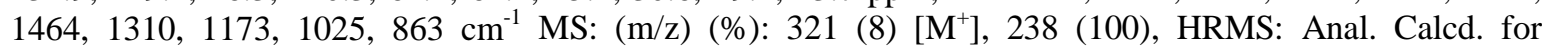
$\mathrm{C}_{16} \mathrm{H}_{22} \mathrm{NO}_{2}{ }^{35} \mathrm{Cl} 295.13391$, Found: 295.13509.

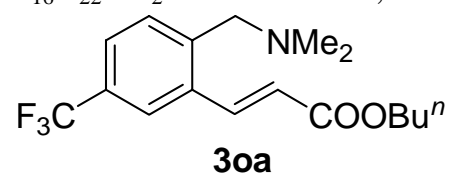

(E)-butyl 3-(2-((dimethylamino)methyl)-5-(trifluoromethyl)phenyl)acrylate (3oa): Start from 10 (102 mg, $0.5 \mathrm{mmol})$ with $2 \mathrm{a}(145 \mu \mathrm{L}, 1.0 \mathrm{mmol})$ to afford 3oa $(123 \mathrm{mg})$, yield $74 \% .{ }^{1} \mathrm{H} \mathrm{NMR}\left(\mathrm{CDCl}_{3}, 300 \mathrm{MHz}\right) \delta 8.13$ $(\mathrm{d}, 1 \mathrm{H}, \mathrm{J}=16.2), 7.79(\mathrm{~s}, 1 \mathrm{H}), 7.53(\mathrm{~d}, 1 \mathrm{H}, \mathrm{J}=7.8), 7.43(\mathrm{~d}, 1 \mathrm{H}, \mathrm{J}=7.8), 6.39(\mathrm{~d}, 1 \mathrm{H}, \mathrm{J}=15.9), 4.19(\mathrm{t}, 2 \mathrm{H}, \mathrm{J}=6.9)$, $3.50(\mathrm{~s}, 2 \mathrm{H}), 2.21(\mathrm{~s}, 6 \mathrm{H}), 1.64(\mathrm{~m}, 2 \mathrm{H}), 1.41(\mathrm{~m}, 2 \mathrm{H}), 0.92(\mathrm{t}, 3 \mathrm{H}, \mathrm{J}=7.2) \mathrm{ppm} ;{ }^{13} \mathrm{C} \mathrm{NMR}\left(\mathrm{CDCl}_{3}, 75 \mathrm{MHz}\right)$ 166.7, 140.8, 136.7, 135.9, 133.4, 131.9, 129.2, 26.3, 120.3, 64.4, 61.1, 45.1, 30.6, 19.1, 13.7 ppm; IR v 2962,

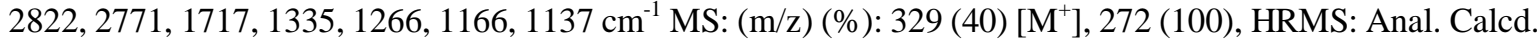
for $\mathrm{C}_{17} \mathrm{H}_{22} \mathrm{NO}_{2} \mathrm{~F}_{3} 329.16026$, Found: 329.16037 .<smiles>COC(=O)/C=C/c1cc2c(cc1CN(C)C)OCO2</smiles><smiles>CCN(C)Cc1ccc2c(c1/C=C/C(=O)OC)OCO2</smiles>

(E)-butyl 3-(6-((dimethylamino)methyl)benzo[d][1,3]dioxol-5-yl)acrylate (3pa) and (E)-butyl 3-(5-((dimethylamino)methyl)benzo[d][1,3]dioxol-4-yl)acrylate (3pa'): Start from 1p (90 mg, $0.5 \mathrm{mmol})$ with 2a (145 $\mu \mathrm{L}, 1.0 \mathrm{mmol})$ to afford 3pa and 3pa' (122 mg, 3pa:3pa' $=12: 1)$, yield $80 \%$. ${ }^{1} \mathrm{H} \mathrm{NMR}\left(\mathrm{CDCl}_{3}\right.$, $300 \mathrm{MHz}) \delta 8.05(\mathrm{~d}, 0.08 \mathrm{H}, \mathrm{J}=15.9), 7.92(\mathrm{~d}, 1.00 \mathrm{H}, \mathrm{J}=16.2), 6.98(\mathrm{~d}, 0.17 \mathrm{H}, \mathrm{J}=9.9), 6.70(\mathrm{~d}, 1.10 \mathrm{H}, 17.1), 6.65$ $(\mathrm{s}, 1.92 \mathrm{H}), 6.15(\mathrm{~d}, 0.19 \mathrm{H}, 15.6), 5.97(\mathrm{~s}, 2.29 \mathrm{H}), 5.90(\mathrm{~s}, 0.38 \mathrm{H}), 4.13(\mathrm{t}, 2.85 \mathrm{H}, \mathrm{J}=6.6), 3.49(\mathrm{~s}, 0.24 \mathrm{H}), 3.31$ $(\mathrm{s}, 2.47 \mathrm{H}), 2.20(\mathrm{~s}, 1.19 \mathrm{H}), 5.15(\mathrm{~s}, 7.31 \mathrm{H}), 1.56(\mathrm{~m}, 2 \mathrm{H}), 1.38(\mathrm{~m}, 2 \mathrm{H}), 0.89(\mathrm{t}, 3 \mathrm{H}, \mathrm{J}=7.5) \mathrm{ppm} ;{ }^{13} \mathrm{C} \mathrm{NMR}$ $\left(\mathrm{CDCl}_{3}, 75 \mathrm{MHz}\right) 167.5,167.3,149.0,147.2,142.1,141.4,137.0,133.6,132.0,128.9,127.6,123.6,122.9$, 121.9, 118.6, 117.7, 116.9, 110.5, 108.2, 106.4, 105.5, 101.3, 64.2, 64.0, 61.8, 61.1, 55.214, 44.8, 44.5, 30.6,

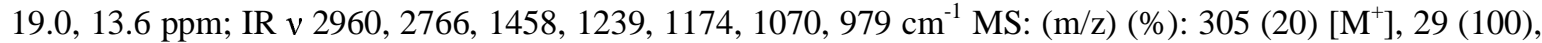
HRMS: Anal. Calcd. for $\mathrm{C}_{17} \mathrm{H}_{23} \mathrm{NO}_{4}$ 305.16271, Found: 305.16328 .<smiles>CNCc1cc2ccccc2cc1/C=C/C(=O)OC</smiles><smiles>CNCc1ccc2ccccc2c1/C=C/C(=O)OC</smiles>

(E)-butyl 3-(3-((dimethylamino)methyl)naphthalen-2-yl)acrylate (3qa) and (E)-butyl 3-(2-((dimethylamino)methyl)naphthalen-1-yl)acrylate (3qa'): Starting from 1q (92 $\mathrm{mg}, 0.5 \mathrm{mmol})$ with 2a $(145 \mu \mathrm{L}, 1.0 \mathrm{mmol})$ to afford 3qa and 3qa' (133 mg, 3qa:3qa'=11:1), yield 85\%. ${ }^{1} \mathrm{H} \mathrm{NMR}\left(\mathrm{CDCl}_{3}\right.$, $300 \mathrm{MHz}) \delta 8.31(\mathrm{~d}, 1 \mathrm{H}, \mathrm{J}=15.9), 8.07(\mathrm{~s}, 1 \mathrm{H}), 7.75(\mathrm{~m}, 2 \mathrm{H}), 7.67(\mathrm{~s}, 1 \mathrm{H}), 7.44(\mathrm{~m}, 2 \mathrm{H}), 6.52(\mathrm{~d}, 1 \mathrm{H}, \mathrm{J}=16.5)$ $4.23(\mathrm{t}, 2 \mathrm{H}), 3.56(\mathrm{~s}, 2 \mathrm{H}), 2.26(\mathrm{~s}, 6 \mathrm{H}), 1.69(\mathrm{~m}, 2 \mathrm{H}), 1.45(\mathrm{~m}, 2 \mathrm{H}), 0.92(\mathrm{t}, 3 \mathrm{H}) \mathrm{ppm} ;{ }^{13} \mathrm{C} \mathrm{NMR}\left(\mathrm{CDCl}_{3}\right.$, $75 \mathrm{MHz}) 167.0,142.8,135.2,133.6,132.7,132.4,129.0,128.0,127.3,126.8,126.3,126.1,119.3,64.2,62.4$, 
45.2, 30.7, 19.1, 13.7 ppm; IR v 2960, 2817, 2767,1712, 1638, 1304, 1171, 1025, $749 \mathrm{~cm}^{-1} \mathrm{MS}:(\mathrm{m} / \mathrm{z})(\%): 311$ (30) $\left[\mathrm{M}^{+}\right], 165$ (100), HRMS: Anal. Calcd. for $\mathrm{C}_{20} \mathrm{H}_{25} \mathrm{NO}_{2}$ 311.18853, Found: 311.18901.<smiles>CN(C)Cc1ccc(Cl)cc1/C=C/C(=O)OCc1ccccc1</smiles>

(E)-benzyl 3-(5-chloro-2-((dimethylamino)methyl)phenyl)acrylate (3nd): Starting from 1n (85 mg, 0.5 mmol) with $2 d(162 \mathrm{mg}, 1.0 \mathrm{mmol})$ to afford 3nd $(136 \mathrm{mg})$, yield $82 \% .{ }^{1} \mathrm{H} \mathrm{NMR}\left(\mathrm{CDCl}_{3}, 300 \mathrm{MHz}\right) \delta 8.13(\mathrm{~d}$, $1 \mathrm{H}, \mathrm{J}=16.2), 7.54(\mathrm{~s}, 1 \mathrm{H}), 7.40-7.22(\mathrm{~m}, 7 \mathrm{H}), 6.38(\mathrm{~d}, 1 \mathrm{H}, \mathrm{J}=15.9), 5.24(\mathrm{~s}, 2 \mathrm{H}), 3.42(\mathrm{~s}, 2 \mathrm{H}), 2.20(\mathrm{~s}, 6 \mathrm{H}) \mathrm{ppm}$; ${ }^{13} \mathrm{C} \mathrm{NMR}\left(\mathrm{CDCl}_{3}, 75 \mathrm{MHz}\right) 166.3,141.4,136.6,135.8,135.7,133.3,131.9,129.3,128.4,128.3,128.1,128.0$, 126.3, 119.8, 66.2, 61.0, 45.0 ppm; IR v 2948, 2818, 2768, 1716, 1636, 1456, 1304, 1166, 1024, 863, 744, 697, $\mathrm{cm}^{-1}$ MS: (m/z) (\%): 329 (1) [M+], 238 (100), HRMS: Anal. Calcd. for $\mathrm{C}_{19} \mathrm{H}_{20} \mathrm{NO}_{2}{ }^{35} \mathrm{Cl}$ 329.11826, Found: 329.11885 .<smiles>CCCCOc1cccc(/C=C/C(=O)OCc2ccccc2)c1CN(C)CC</smiles>

(E)-benzyl 3-(3-butoxy-2-((dimethylamino)methyl)phenyl)acrylate (3rd): Starting from 1r (104 mg, 0.5 mmol) with $2 d(162 \mathrm{mg}, 1.0 \mathrm{mmol})$ to afford 3rd $(148 \mathrm{mg})$, yield $81 \% .{ }^{1} \mathrm{H} \mathrm{NMR}\left(\mathrm{CDCl}_{3}, 300 \mathrm{MHz}\right) \delta 8.26(\mathrm{~d}$, $1 \mathrm{H}, \mathrm{J}=15.9), 7.43-7.20(\mathrm{~m}, 7 \mathrm{H}), 6.86(\mathrm{~m}, 1 \mathrm{H}), 6.43(\mathrm{~d}, 1 \mathrm{H}, \mathrm{J}=16.2), 5.24(\mathrm{~s}, 2 \mathrm{H}), 3.71(\mathrm{~d}, 2 \mathrm{H}, \mathrm{J}=6.6), 3.575(\mathrm{~s}$, $2 \mathrm{H}), 2.24(\mathrm{~s}, 6 \mathrm{H}), 2.12(\mathrm{~m}, 1 \mathrm{H}), 1.05(\mathrm{~d}, 6 \mathrm{H}, \mathrm{J}=6.6) \mathrm{ppm} ;{ }^{13} \mathrm{C} \mathrm{NMR}\left(\mathrm{CDCl}_{3}, 75 \mathrm{MHz}\right) 166.8,157.7,143.5$, 136.1, 135.9, 128.5, 128.4, 128.1, 128.1, 127.2, 118.8, 118.4, 112.5, 74.6, 52.5, 45.3, 28.4, 19.4 ppm; IR v

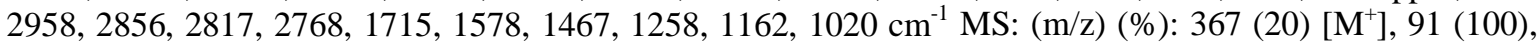
HRMS: Anal. Calcd. for $\mathrm{C}_{23} \mathrm{H}_{29} \mathrm{NO}_{3} \mathrm{~N}$ 367.21474, Found: 367.21367.

Transforamtion from Functionalized Tertiary Amines to Toluene Derivatives (Eq. 4).<smiles></smiles>

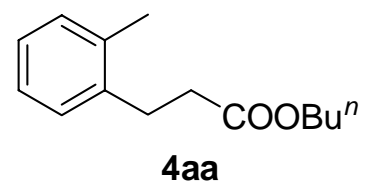

butyl 3-o-tolylpropanoate (4aa): 4aa (118 mg) was obtained from 3aa $(130 \mathrm{mg}, 0.5 \mathrm{mmol})$ with a reaction time of $8 \mathrm{~h}$, yield 81\%. ${ }^{1} \mathrm{H} \mathrm{NMR}\left(\mathrm{CDCl}_{3}, 300 \mathrm{MHz}\right) \delta 7.14-7.12(\mathrm{~m}, 4 \mathrm{H}), 4.09(\mathrm{t}, 2 \mathrm{H}, \mathrm{J}=6.6), 2.95(\mathrm{t}, 2 \mathrm{H}, \mathrm{J}=7.5)$, 2.59 (t, 2H, J=7.5), $2.33(\mathrm{~s}, 3 \mathrm{H}), 1.60(\mathrm{~m}, 2 \mathrm{H}), 1.35(\mathrm{~m}, 2 \mathrm{H}), 0.93(\mathrm{t}, 3 \mathrm{H}, \mathrm{J}=6.9) \mathrm{ppm} ;{ }^{13} \mathrm{C} \mathrm{NMR}\left(\mathrm{CDCl}_{3}\right.$, $75 \mathrm{MHz}) 173.1,136.6,135.9,130.2,130.2,128.4,126.3,126.0,64.3,34.5,30.6,28.3,19.2,13.6 \mathrm{ppm}$; IR v 2960, 1735, 1176, $750 \mathrm{~cm}^{-1}$ MS: (m/z) (\%): $220(40)\left[\mathrm{M}^{+}\right], 105$ (100).<smiles>CCCCCCc1ccc(C)cc1C</smiles><smiles>CCCCCCC(=O)OCCCc1c(C)cccc1C</smiles>

butyl 3-(2,4-dimethylphenyl)propanoate (4ha) and butyl 3-(2,6-dimethylphenyl) propanoate (4ha'): 4ha and 4ha' (57 mg, 4ha:4ha'=9:1) was obtained from 3ha and 3ha' (75 mg, $0.27 \mathrm{mmol}$, 3ha:3ha'=9:1) with a reaction time of $8 \mathrm{~h}$, yield $90 \% .{ }^{1} \mathrm{H}$ NMR $\left(\mathrm{CDCl}_{3}, 300 \mathrm{MHz}\right) \delta 7.05-7.00(\mathrm{~m}, 3 \mathrm{H}), 4.10(\mathrm{t}, 2 \mathrm{H}, \mathrm{J}=6.6), 2.92(\mathrm{t}$, $2 \mathrm{H}, \mathrm{J}=7.5), 2.58(\mathrm{t}, 2 \mathrm{H}, \mathrm{J}=7.5), 2.29(\mathrm{~s}, \mathrm{~s}, 6 \mathrm{H}), 1.61(\mathrm{~m}, 2 \mathrm{H}), 1.38(\mathrm{~m}, 2 \mathrm{H}), 0.95(\mathrm{t}, 3 \mathrm{H}, \mathrm{J}=7.4) \mathrm{ppm} ;{ }^{13} \mathrm{C} \mathrm{NMR}$ $\left(\mathrm{CDCl}_{3}, 75 \mathrm{MHz}\right) 173.1(\mathrm{~h}), 136.0,135.64,135.59,135.5,131.0(\mathrm{~h}), 128.3(\mathrm{~h}), 128.1,126.6,126.0,64.3,64.2(\mathrm{~h})$, 34.7(h), 34.4, 33.3, 30.5(h), 27.9(h), 25.0, 20.7(h), 19.6, 19.0(h), 13.6(h) ppm; IR v 2960, 1736, 1172, $819 \mathrm{~cm}^{-1}$ 
MS: (m/z) (\%): $234(30)\left[\mathrm{M}^{+}\right], 119(100)$.

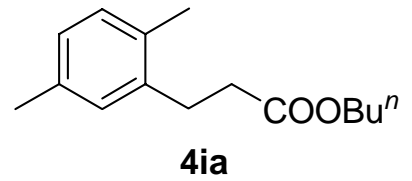

butyl 3-(2,5-dimethylphenyl)propanoate (4ia): 4ia (52 mg) was obtained from 3ia (66 mg, $0.24 \mathrm{mmol}$ ) with a reaction time of $8 \mathrm{~h}$, yield $90 \% .{ }^{1} \mathrm{H}$ NMR $\left(\mathrm{CDCl}_{3}, 300 \mathrm{MHz}\right) \delta 7.02(\mathrm{~d}, 1 \mathrm{H}, \mathrm{J}=7.8), 6.94(\mathrm{~m}, 2 \mathrm{H}), 4.09(\mathrm{t}, 2 \mathrm{H}$, $\mathrm{J}=6.9), 2.90(\mathrm{t}, 2 \mathrm{H}, \mathrm{J}=7.8), 2.57(\mathrm{t}, 2 \mathrm{H}, \mathrm{J}=8.7), 2.28(\mathrm{~s}, \mathrm{~s}, 6 \mathrm{H}), 1.60(\mathrm{~m}, 2 \mathrm{H}), 1.35(\mathrm{~m}, 2 \mathrm{H}), 0.92(\mathrm{t}, 3 \mathrm{H}, \mathrm{J}=6.9)$ ppm; ${ }^{13} \mathrm{C}$ NMR $\left(\mathrm{CDCl}_{3}, 75 \mathrm{MHz}\right) 173.1,138.4,135.4,132.6,130.1,138.2,136.9,64.3,34.6,30.6,28.3,20.9$, 19.0, 18.7, 13.6 ppm; IR v 2960, 1736, 1468, 1171, $810 \mathrm{~cm}^{-1} \mathrm{MS}:(\mathrm{m} / \mathrm{z})(\%): 234$ (50) [M+1, 119 (100), HRMS: Anal. Calcd. for $\mathrm{C}_{15} \mathrm{H}_{22} \mathrm{O}_{2} 234.16198$, Found: 234.16181.<smiles>COC(=O)CCc1cccc(OC)c1C</smiles>

butyl 3-(3-methoxy-2-methylphenyl)propanoate (4ja): 4ja (48 $\mathrm{mg})$ was obtained from $\mathbf{3 j a}$ (63 $\mathrm{mg}, 0.22$ mmol) with a reaction time of $20 \mathrm{~h}$, yield $89 \%$. ${ }^{1} \mathrm{H} \mathrm{NMR}\left(\mathrm{CDCl}_{3}, 300 \mathrm{MHz}\right) \delta 7.02(\mathrm{t}, 1 \mathrm{H}, \mathrm{J}=7.8), 6.69(\mathrm{~m}, 2 \mathrm{H})$, $4.01(\mathrm{t}, 2 \mathrm{H}, \mathrm{J}=6.9), 3.74(\mathrm{~s}, 3 \mathrm{H}), 2.89(\mathrm{t}, 2 \mathrm{H}, \mathrm{J}=7.8), 2.49(\mathrm{t}, 2 \mathrm{H}, \mathrm{J}=8.4), 2.12(\mathrm{~s}, 3 \mathrm{H}), 1.53(\mathrm{~m}, 2 \mathrm{H}), 1.29(\mathrm{~m}$, $2 \mathrm{H}), 0.86(\mathrm{t}, 3 \mathrm{H}, \mathrm{J}=7.8) \mathrm{ppm} ;{ }^{13} \mathrm{C} \mathrm{NMR}\left(\mathrm{CDCl}_{3}, 75 \mathrm{MHz}\right) 173.1,157.7,140.0,131.0,127.8,114.3,111.2,64.3$, 55.1, 34.5, 30.6, 28.5, 19.0, 18.2, 13.6 ppm; IR v 2958, 1734, 1468, 1258, 1176, 1102, $780 \mathrm{~cm}^{-1} \mathrm{MS}:(\mathrm{m} / \mathrm{z})(\%)$ : $234(50)\left[\mathrm{M}^{+}\right], 119(100)$.

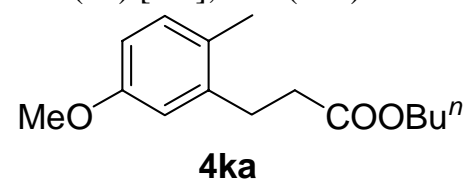

butyl 3-(5-methoxy-2-methylphenyl)propanoate(4ka): 4ka (57 $\mathrm{mg})$ was obtained from 3ka $(81 \mathrm{mg}, 0.28$ mmol) with a reaction time of $8 \mathrm{~h}$, yield $82 \% .{ }^{1} \mathrm{H} \mathrm{NMR}\left(\mathrm{CDCl}_{3}, 300 \mathrm{MHz}\right) \delta 7.02(\mathrm{~d}, 1 \mathrm{H}, \mathrm{J}=8.4), 6.67(\mathrm{~m}, 2 \mathrm{H})$, $4.07(\mathrm{t}, 2 \mathrm{H}, \mathrm{J}=6.6), 3.75(\mathrm{~s}, 3 \mathrm{H}), 2.89(\mathrm{t}, 2 \mathrm{H}, \mathrm{J}=7.5), 2.55(\mathrm{t}, 2 \mathrm{H}, \mathrm{J}=8.0), 2.23(\mathrm{~s}, 3 \mathrm{H}), 1.58(\mathrm{~m}, 2 \mathrm{H}), 1.33(\mathrm{~m}$, $2 \mathrm{H}), 0.91(\mathrm{t}, 3 \mathrm{H}, \mathrm{J}=7.2) \mathrm{ppm} ;{ }^{13} \mathrm{C} \mathrm{NMR}\left(\mathrm{CDCl}_{3}, 75 \mathrm{MHz}\right) 173.0,157.8,140.0,126.1,124.5,121.1,108.2,64.3$, 55.4, 34.9, 30.6, 28.7, 19.1, 13.7, $11.1 \mathrm{ppm}$; IR $v 2959,1734,1504,1252,1162,1047,812 \mathrm{~cm}^{-1} \mathrm{MS}:(\mathrm{m} / \mathrm{z})(\%)$ : $250(100)\left[\mathrm{M}^{+}\right]$.

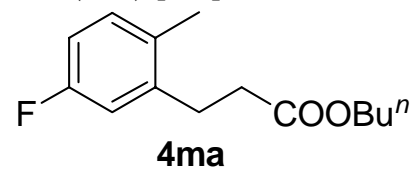

butyl 3-(5-fluoro-2-methylphenyl)propanoate (4ma): 4ma $\quad(40 \quad \mathrm{mg})$ and methyl 3-(5-fluoro-2-methylphenyl)propanoate (4mc) $(11 \mathrm{mg})$ was obtained from 3ma $(96 \mathrm{mg}, 0.34 \mathrm{mmol})$ with a reaction time of $36 \mathrm{~h}$, yield $67 \% .{ }^{1} \mathrm{H} \mathrm{NMR}\left(\mathrm{CDCl}_{3}, 300 \mathrm{MHz}\right) \delta 7.10(\mathrm{~m}, 1 \mathrm{H}), 6.86-6.77(\mathrm{~m}, 2 \mathrm{H}), 4.08(\mathrm{t}, 2 \mathrm{H}$, $\mathrm{J}=6.9), 2.91(\mathrm{t}, 2 \mathrm{H}, \mathrm{J}=7.2), 2.57(\mathrm{t}, 2 \mathrm{H}, \mathrm{J}=8.4), 2.27(\mathrm{~s}, 3 \mathrm{H}), 1.62(\mathrm{~m}, 2 \mathrm{H}), 1.35(\mathrm{~m}, 2 \mathrm{H}), 0.92(\mathrm{t}, 3 \mathrm{H}, \mathrm{J}=6.9)$ $\mathrm{ppm} ;{ }^{13} \mathrm{C} \mathrm{NMR}\left(\mathrm{CDCl}_{3}, 75 \mathrm{MHz}\right) 172.8,162.8,159.6,140.6$ (d, J=6.4), 131.4, 131.2, 115.0 (d, J=21.4), 112.8 (d, J=20.2), 64.4, 34.1, 30.6, 28.3, 28.2, 19.1, 18.5, 13.6 ppm; IR v 2961, 2935, 2874, 1735, 1499, 1458, 1248 , 1178, $810 \mathrm{~cm}^{-1}$ MS: (m/z) (\%): 238 (60) [ $\left.\mathrm{M}^{+}\right], 123$ (100), HRMS: Anal. Calcd. for $\mathrm{C}_{14} \mathrm{H}_{19} \mathrm{O}_{2} \mathrm{~F}$ 238.13691, Found: 238.13686

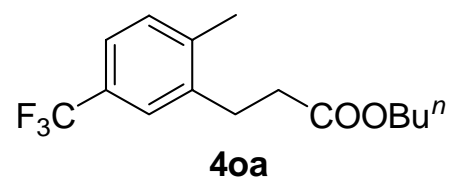

butyl 3-(2-methyl-5-(trifluoromethyl)phenyl)propanoate (4oa): 4oa (85 mg) was obtained from 3oa (98 $\mathrm{mg}, 0.30 \mathrm{mmol})$ with a reaction time of $48 \mathrm{~h}$, yield $47 \% .{ }^{1} \mathrm{H} \mathrm{NMR}\left(\mathrm{CDCl}_{3}, 300 \mathrm{MHz}\right) \delta 7.30(\mathrm{~d}, 2 \mathrm{H}, \mathrm{J}=8.4)$, 7.17 (d, 1H, J=7.5), 4.01 (t, 2H, J=6.6), 2.91 (t, 2H, J=7.2), 2.54 (t, 2H, J=7.8), 2.30 (s, 3H), 1.53 (m, 2H), 1.27 $(\mathrm{m}, 2 \mathrm{H}), 0.85$ (t, 3H, J=7.2) ppm; ${ }^{13} \mathrm{C} \mathrm{NMR}\left(\mathrm{CDCl}_{3}, 75 \mathrm{MHz}\right) 172.6,140.2,139.4,130.5,125.2,125.1,125.0$, 
123.13, 123.09, 64.5, 34.2, 30.6, 28.1, 19.3, 19.0, 13.6 ppm; IR v 2962, 1736, 1330, 1161, 1130, $826 \mathrm{~cm}^{-1} \mathrm{MS}$ : $(\mathrm{m} / \mathrm{z})(\%): 288$ (30) $\left[\mathrm{M}^{+}\right], 173$ (100), HRMS: Anal. Calcd. for $\mathrm{C}_{15} \mathrm{H}_{19} \mathrm{O}_{2} \mathrm{~F}_{3}$ 288.13371, Found: 288.13329.

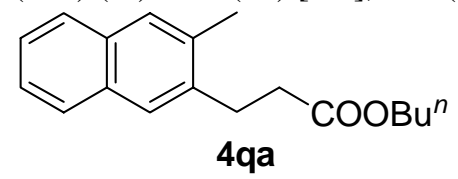

butyl 3-(3-methylnaphthalen-2-yl)propanoate (4qa): 4qa (55 mg) was obtained from 3qa (64 mg, 0.20 $\mathrm{mmol})$ with a reaction time of $48 \mathrm{~h}$, yield $66 \% .{ }^{1} \mathrm{H} \mathrm{NMR}\left(\mathrm{CDCl}_{3}, 300 \mathrm{MHz}\right) \delta 7.73(\mathrm{~m}, 2 \mathrm{H}), 7.60(\mathrm{~m}, 2 \mathrm{H}), 7.39$ $(\mathrm{m}, 2 \mathrm{H}), 4.10(\mathrm{t}, 2 \mathrm{H}, \mathrm{J}=6.6), 3.10(\mathrm{t}, 2 \mathrm{H}, \mathrm{J}=7.5), 2.70(\mathrm{t}, 2 \mathrm{H}, \mathrm{J}=7.8), 2.50(\mathrm{~s}, 3 \mathrm{H}), 1.60(\mathrm{~m}, 2 \mathrm{H}), 1.31(\mathrm{~m}, 2 \mathrm{H})$, 0.90 (t, 3H, J=7.5) ppm; ${ }^{13} \mathrm{C}$ NMR $\left(\mathrm{CDCl}_{3}, 75 \mathrm{MHz}\right) 173.1,137.5,134.6,132.4,132.2,128.2,128.1,127.1$, $126.8,125.4,125.3,125.11,125.08, \quad 64.4,34.5,30.6,28.4,19.7,19.1,13.6$ ppm; IR v 2960, 1735, 1173, 747 $\mathrm{cm}^{-1}$ MS: (m/z) (\%): $270(55)\left[\mathrm{M}^{+}\right], 155$ (100), HRMS: Anal. Calcd. for $\mathrm{C}_{18} \mathrm{H}_{22} \mathrm{O}_{2}$ 270.16198, Found: 270.16159.

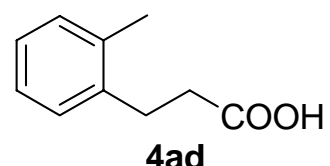

3-o-tolylpropanoic acid (4ad): Follow the general procedure for reduction of functionalized amine to substituted toluene from the starting $3 \mathbf{a d}(79 \mathrm{mg}, 0.27 \mathrm{mmol})$ with a reaction time of $12 \mathrm{~h}$, after the evaporation of $\mathrm{MeOH}$, the residue was dissolved in $\mathrm{CH}_{2} \mathrm{Cl}_{2}$ and washed with $\mathrm{HCl}(2 \mathrm{~N})$, the water phase was extracted by $\mathrm{CH}_{2} \mathrm{Cl}_{2}$ twice, the organic layer was combined, dried over $\mathrm{Na}_{2} \mathrm{SO}_{4}$, and evaporated to give $4 \mathbf{a d}(37.7 \mathrm{mg})$ as a white needle crystal, yield $77 \%$. m. p. 104-106 $\square ;{ }^{1} \mathrm{H}$ NMR $\left(\mathrm{CDCl}_{3}, 300 \mathrm{MHz}\right) \delta 11.3(\mathrm{br}, 1 \mathrm{H}), 7.15(\mathrm{~m}, 4 \mathrm{H})$, $2.96(\mathrm{t}, 2 \mathrm{H}, \mathrm{J}=7.8), 2.65(\mathrm{t}, 2 \mathrm{H}, \mathrm{J}=8.0), 2.33(\mathrm{~s}, 3 \mathrm{H}) \mathrm{ppm} ;{ }^{13} \mathrm{C} \mathrm{NMR}\left(\mathrm{CDCl}_{3}, 75 \mathrm{MHz}\right) 179.5,138.2,135.9$, 130.3, 128.4, 126.5, 126.2, 34.3, 27.9, 19.2 ppm; IR v 3394, 2927, 2470, 1718, 1461, 1261, 1159, 1024, 745 $\mathrm{cm}^{-1}$ MS: (m/z) (\%): $162(50)\left[\mathrm{M}^{+}\right], 105(100)$.<smiles>CCCCOc1cccc(CCC(=O)O)c1C</smiles>

3-(3-isobutoxy-2-methylphenyl)propanoic acid (4rd): Follow the procedure of 4ad, 4rd $(57.0 \mathrm{mg})$ was obtained as a white needle crystal from the starting 3rd $(110 \mathrm{mg}, 0.3 \mathrm{mmol})$ in 2-methoxyethanol $(3.0 \mathrm{~mL})$ with a reaction time of $22 \mathrm{~h}$, yield $81 \%$. m. p. 105-107 $\square$; ${ }^{1} \mathrm{H}$ NMR $\left(\mathrm{CDCl}_{3}, 300 \mathrm{MHz}\right) \delta 7.07(\mathrm{t}, 1 \mathrm{H}, \mathrm{J}=8.1)$, 6.77-6.68 (m, 2H) $3.69(\mathrm{~d}, 2 \mathrm{H}, \mathrm{J}=6.3) 2.95(\mathrm{t}, 2 \mathrm{H}, \mathrm{J}=7.8), 2.61(\mathrm{t}, 2 \mathrm{H}, \mathrm{J}=8.7), 2.20(\mathrm{~s}, 3 \mathrm{H}) 2.08(\mathrm{~m}, 1 \mathrm{H}) 1.03(\mathrm{~d}$, $6 \mathrm{H}, \mathrm{J}=6.9) \mathrm{ppm} ;{ }^{13} \mathrm{C} \mathrm{NMR}\left(\mathrm{CDCl}_{3}, 75 \mathrm{MHz}\right) 178.8,157.3,139.5,126.2,124.8,120.7,109.2,74.4,34.5,28.44$, 28.40, 19.3, $11.2 \mathrm{ppm}$; IR v 2957, 2926, 2873, 1740, 1462, 1260, 1173, 1096, $778 \mathrm{~cm}^{-1} \mathrm{MS}:(\mathrm{m} / \mathrm{z})(\%): 236(10)$ $\left[\mathrm{M}^{+}\right], 134(100)$.

\section{Mechanistic Investigation}

Ortho Olefination of Palladacycle 5 (Eq. 5)

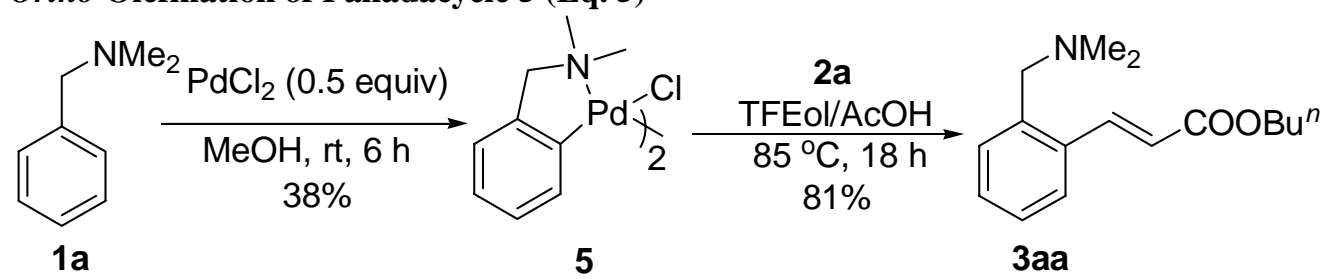

Preparation of $\boldsymbol{\mu}$-(Dichloro)-bis(N, N-dimethylbenzylamine-C, N)dipalladium (5): The Pd-complex was prepared according to the literature ${ }^{1}$ from $1 \mathbf{a}(270 \mathrm{mg}, 2 \mathrm{mmol})$, and palladium dichloride (177 $\left.\mathrm{mg}, 1 \mathrm{mmol}\right)$ in methanol at RT, recrystallization in benzene and hexane give $5(106 \mathrm{mg}, 38 \%)$. m. p. 184-186 $\square$; ${ }^{1} \mathrm{H} \mathrm{NMR}$ $\left(\mathrm{CDCl}_{3}, 300 \mathrm{MHz}\right) \delta 6.85-7.24(\mathrm{~m}, 4 \mathrm{H}), 3.91(\mathrm{~s}, 2 \mathrm{H}), 2.83(\mathrm{~d}, 6 \mathrm{H}, \mathrm{J}=7.8) \mathrm{ppm} ;{ }^{13} \mathrm{C} \mathrm{NMR}\left(\mathrm{CDCl}_{3}, 75 \mathrm{MHz}\right)$ 
146.9, 143.0, 133.3, 132.8, 125.1, 124., 121.5, 73.2, 73.1, 52.8, 52.5 ppm; IR v 3002, 2911, 1578, 1448, 1286, 1044, 1026, 983, 861, $734 \mathrm{~cm}^{-1}$ MS: (m/z) (\%): $552(6)\left[\mathrm{M}^{+}\right], 134(100)$.

Procedure from 5 to 3aa: $5(55.2 \mathrm{mg}, 0.1 \mathrm{mmol})$ was dissolved in $\mathrm{CF}_{3} \mathrm{CH}_{2} \mathrm{OH}(0.4 \mathrm{~mL})$ in a Schlenck tube. Then $2 \mathrm{a}(29 \mu \mathrm{L}, 0.2 \mathrm{mmol})$ and $\mathrm{HOAc}(0.1 \mathrm{ml}, 3.2 \mathrm{mmol})$ were added. The flask was stoppered and heated at $85^{\circ} \mathrm{C}$ in an oil bath for $18 \mathrm{~h}$. Work up was the same as the general procedure and afforded 3aa $(42.1 \mathrm{mg}, 81 \%)$.

Determination of Kinetic Isotope Effect $\left(k_{\mathrm{H}} / k_{\mathrm{D}}\right)($ Eq. 6)

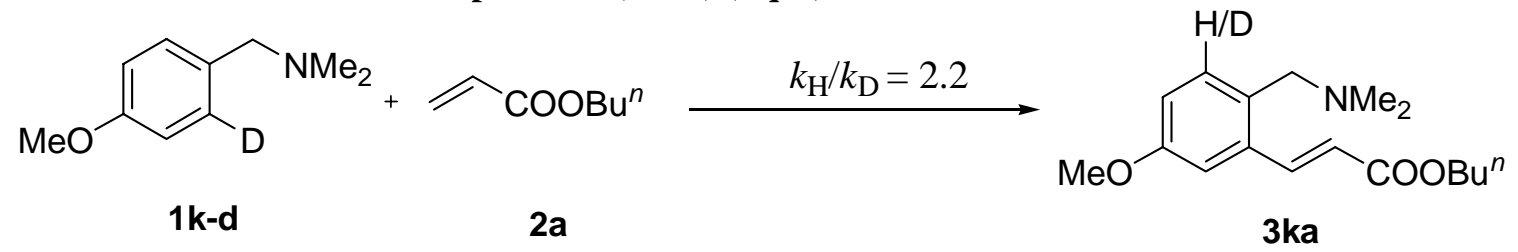

2-Deuterated $\mathbf{N}, \mathbf{N}$-dimethyl-4-methoxyl- benzylamine (1k-d): $1 \mathrm{k}-\mathrm{d}$ was prepared from $1 \mathbf{k}$ according to the literature. ${ }^{2}$ In a dried Schlenck tube were combined $1 \mathbf{k}(330 \mathrm{mg}, 2 \mathrm{mmol})$, dried ether $(3 \mathrm{~mL})$ and $n$-BuLi (2.5 $\mathrm{mL}, 4 \mathrm{mmol})$, tightly soppered, and stirred at RT for $22 \mathrm{~h}$. Then $\mathrm{D}_{2} \mathrm{O}(0.5 \mathrm{~mL}, 2.2 \mathrm{mmol})$ was added with an ice bath. The mixture was stirred at RT for $1 \mathrm{~h}$. After that, the organic layer was filtered free of the damp solid which had separated, dried over $\mathrm{MgSO}_{4}$, and evaporated the solvent to get $\mathbf{1 k}$-d quantitatively (deuterated rate 79\%).

Procedure of determination of isotope effect: Olefination of $1 \mathrm{k}-\mathrm{d}(84 \mathrm{mg}, 0.5 \mathrm{mmol})$ according to the general procedure afforded 3ka (106 mg, yield 73\%, deuterated rate $48 \%)$. The kinetic isotope effect $\left(k_{\mathrm{H}} / k_{\mathrm{D}}\right)$ was determined to be 2.2 .

\section{References:}

1. Cope, A. C.; Friedrich, E. C. J. Am. Chem. Soc. 1968, 90, 909.

2. Jones, F. N.; Zinn, M. F.; Hauser, C. R. J. Org. Chem. 1963, 28, 663. 
NMR spectrum of compounds $\mathbf{1 r}$<smiles>CCCCOc1ccccc1CN(C)C</smiles>
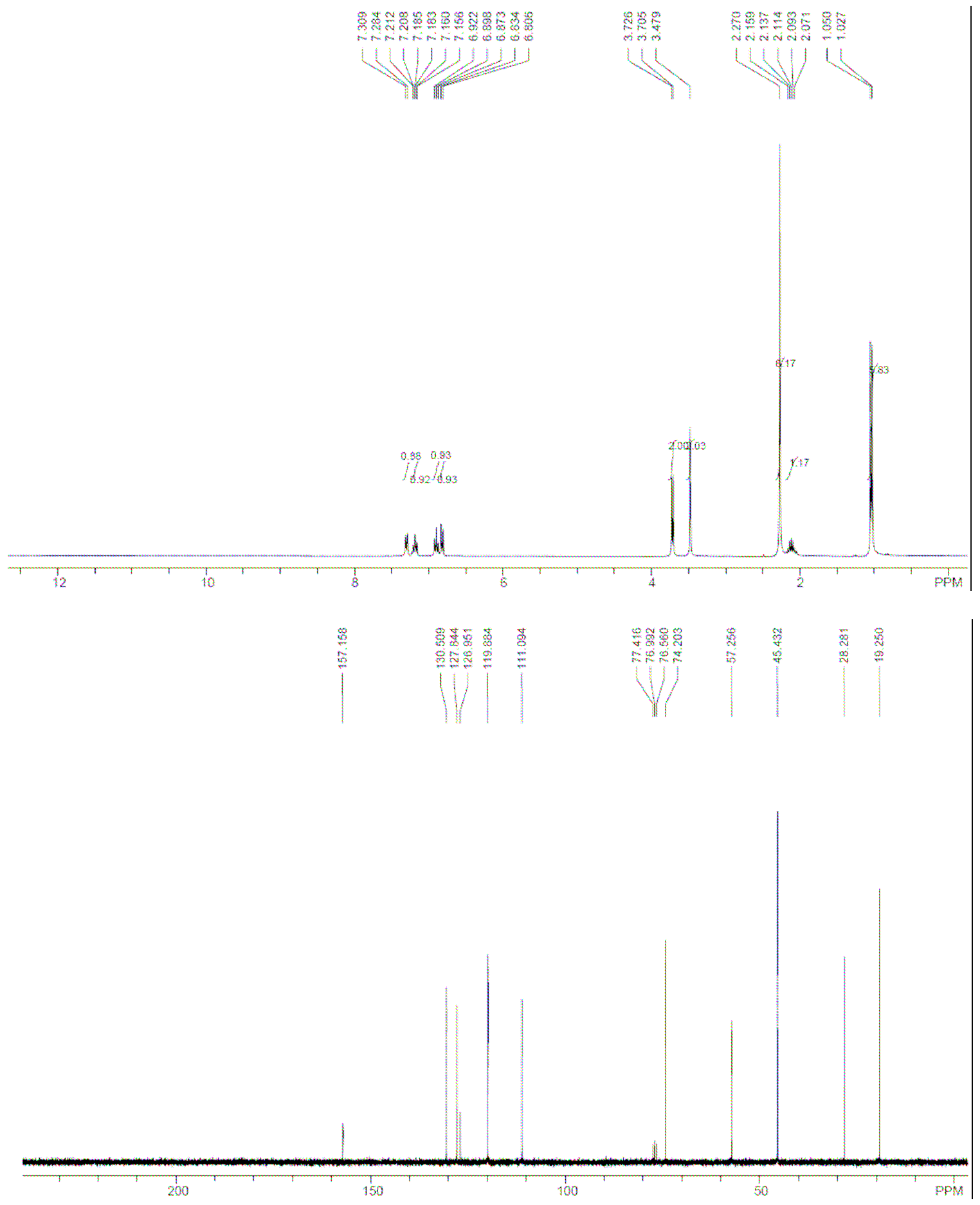
NMR spectrum of compounds 3aa-3rd
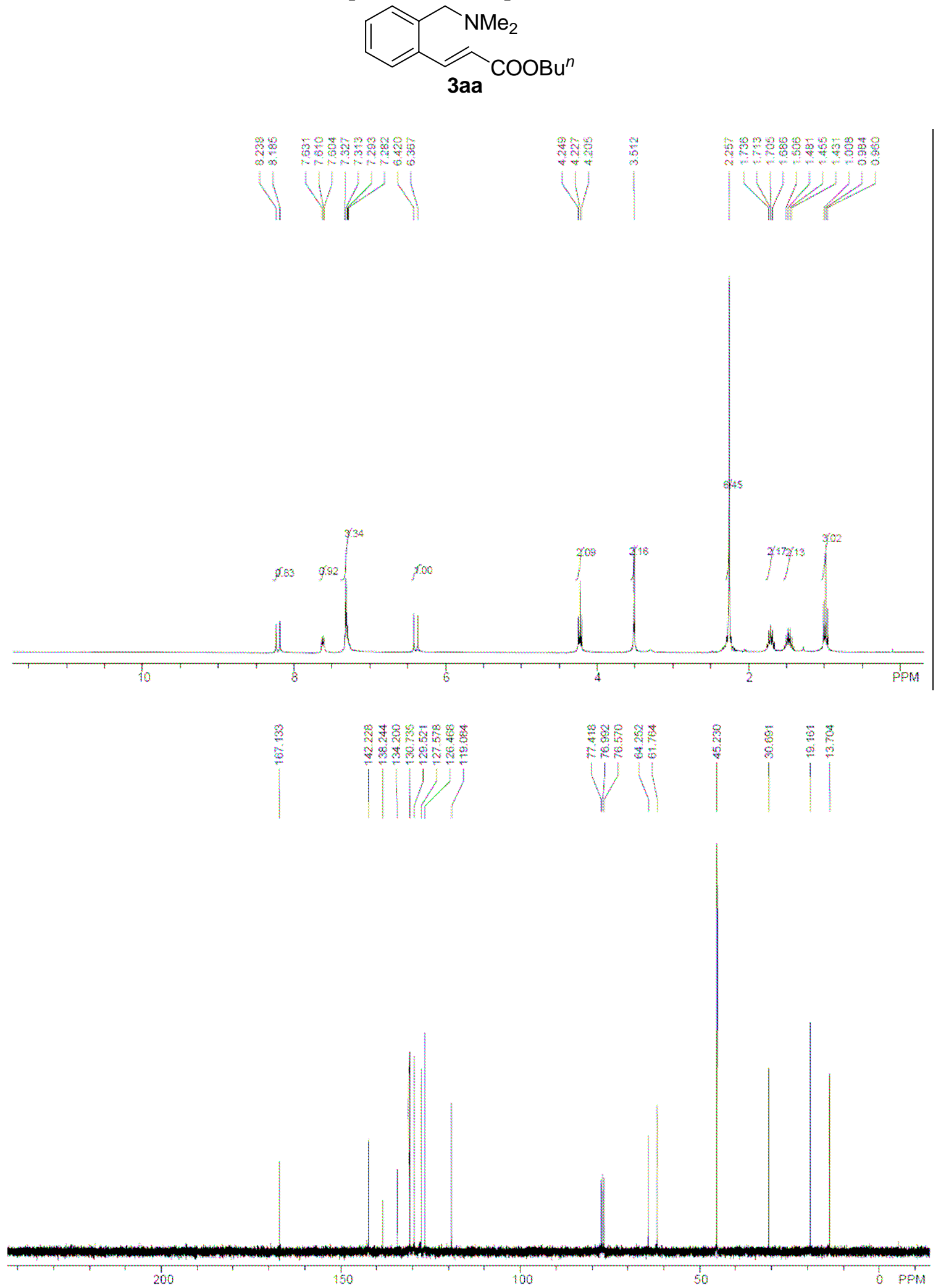

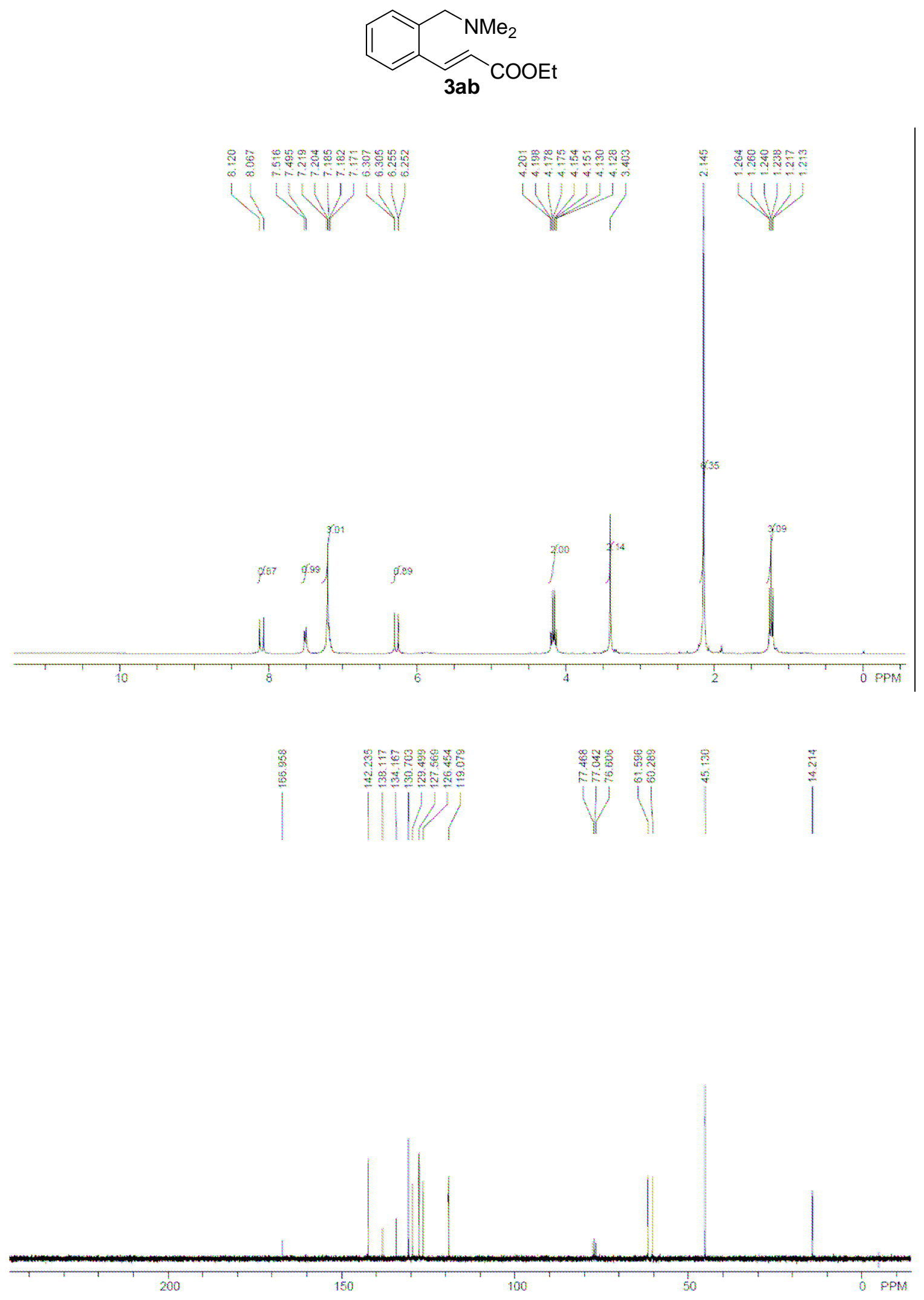

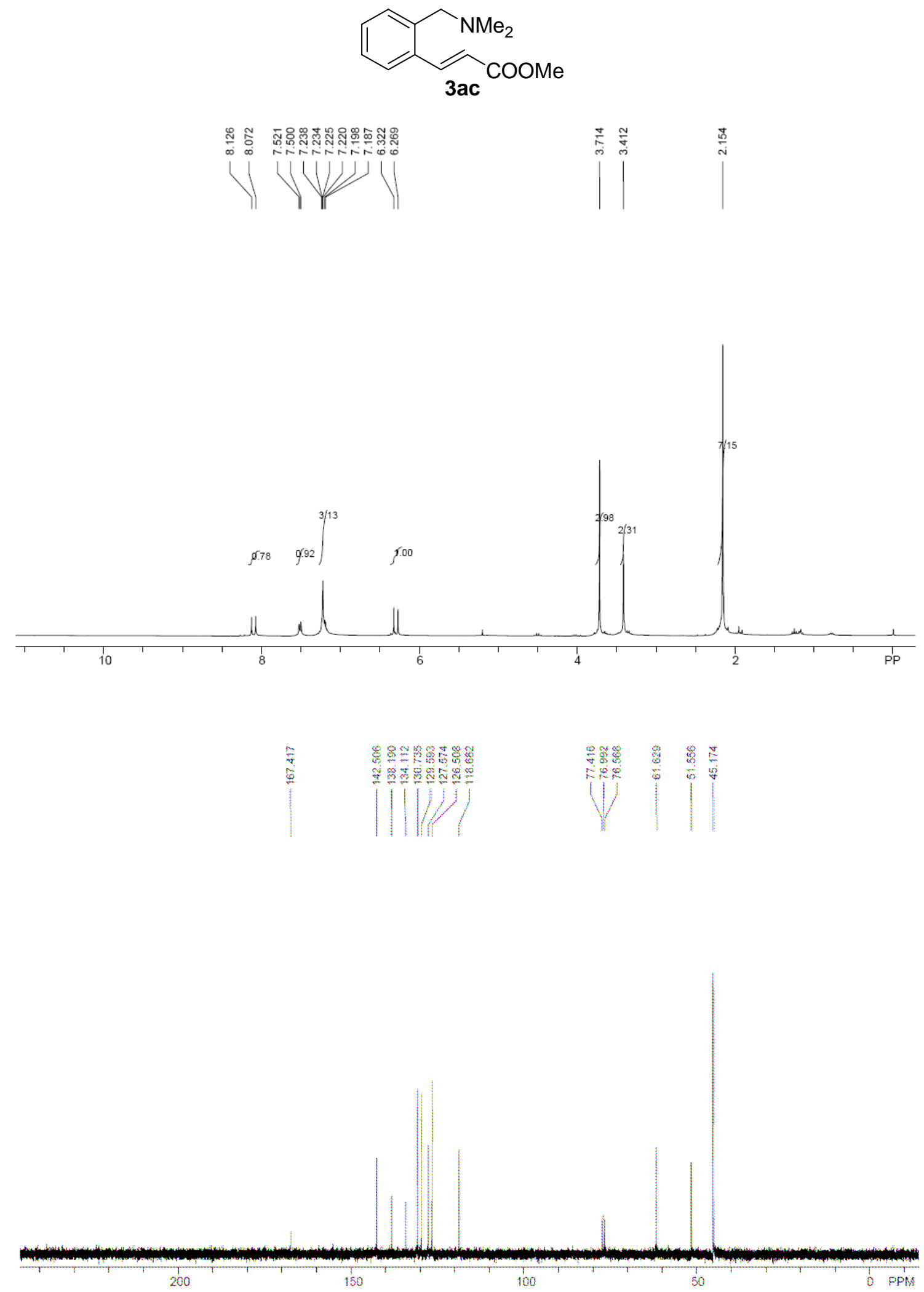

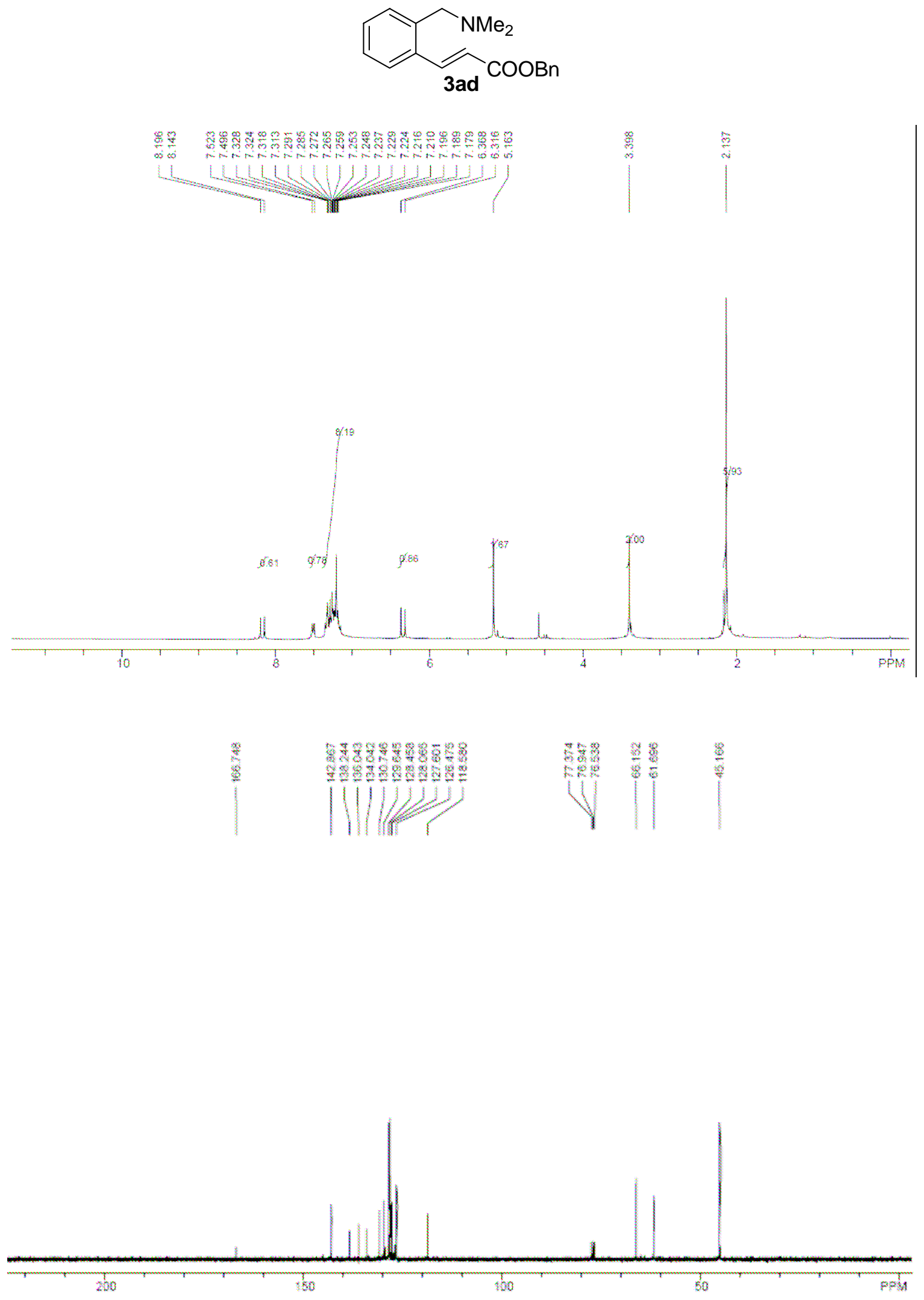

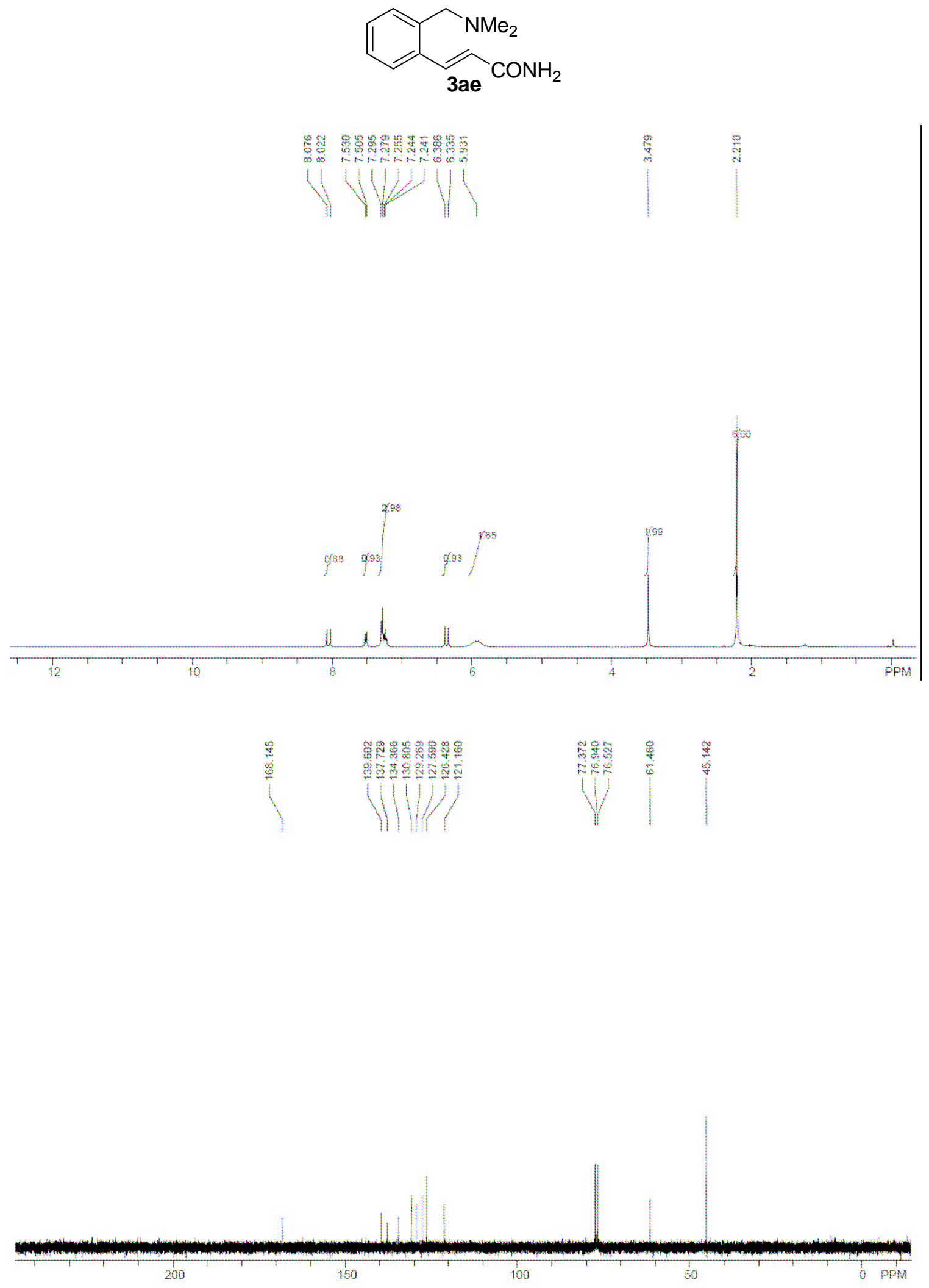


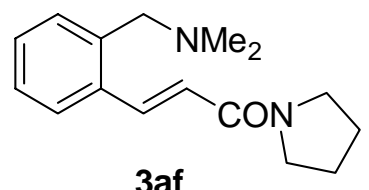

3af

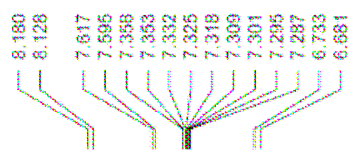

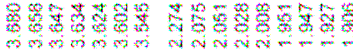

(l)
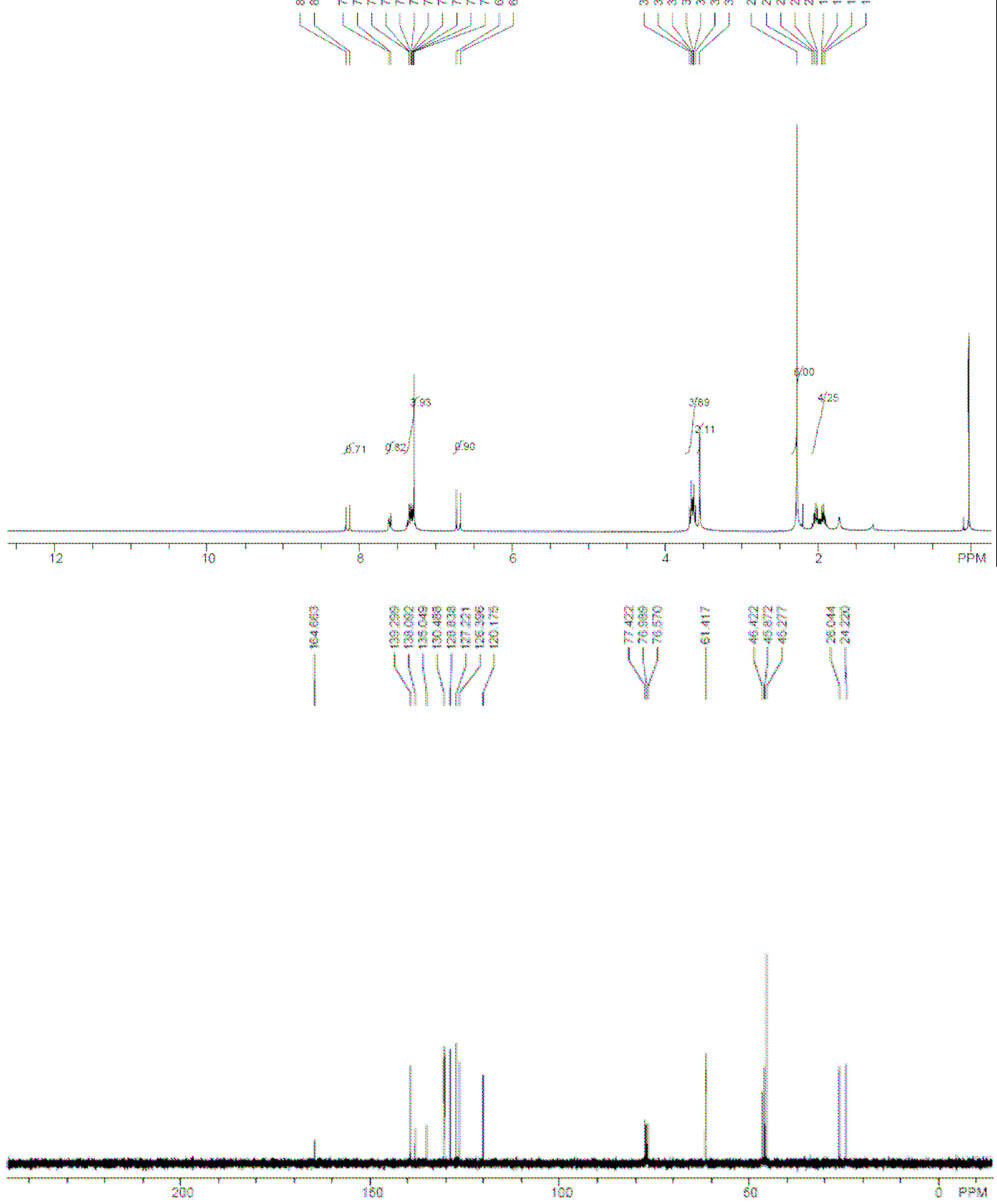

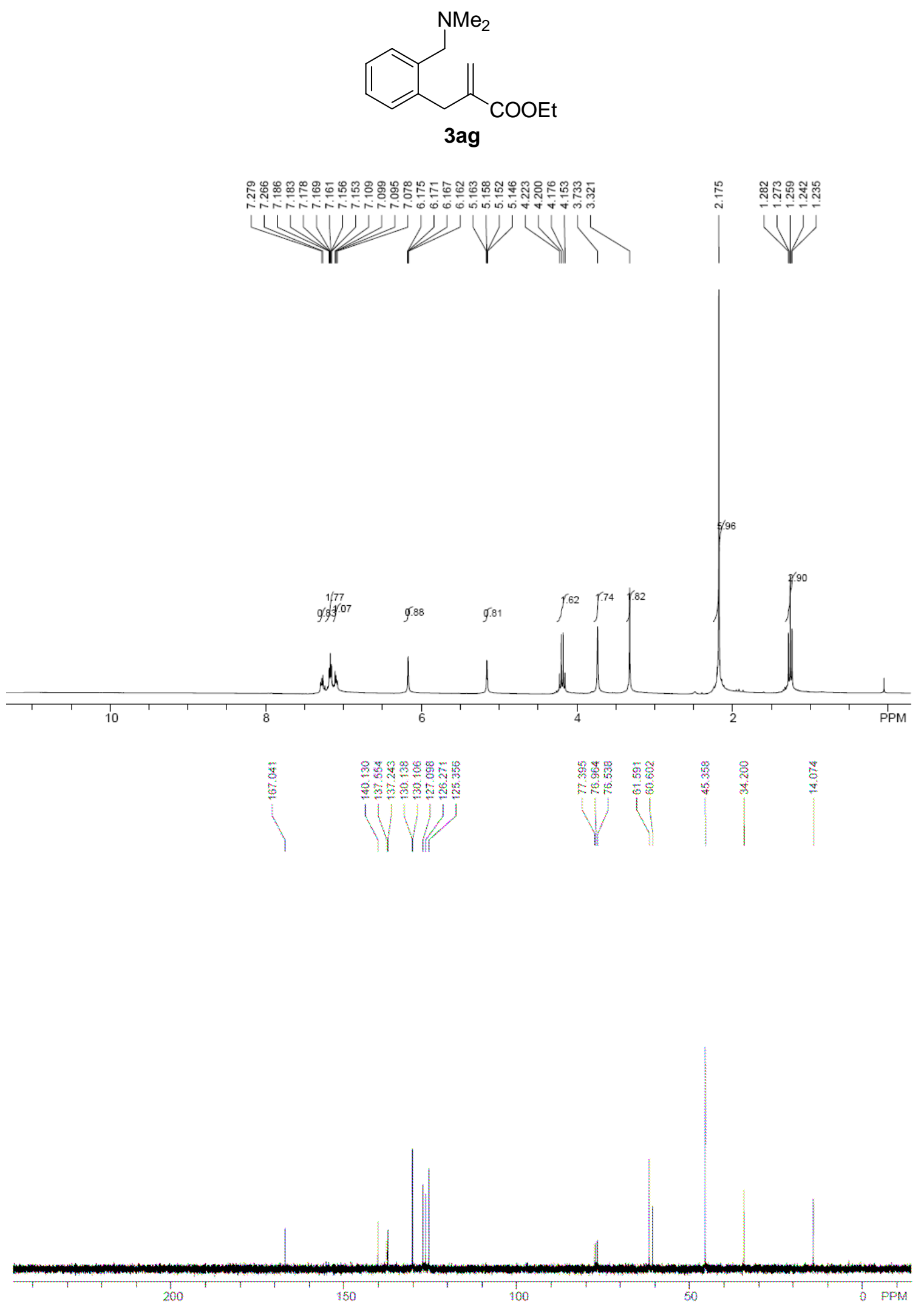
NMR spectrum of compounds 3ga-3rd
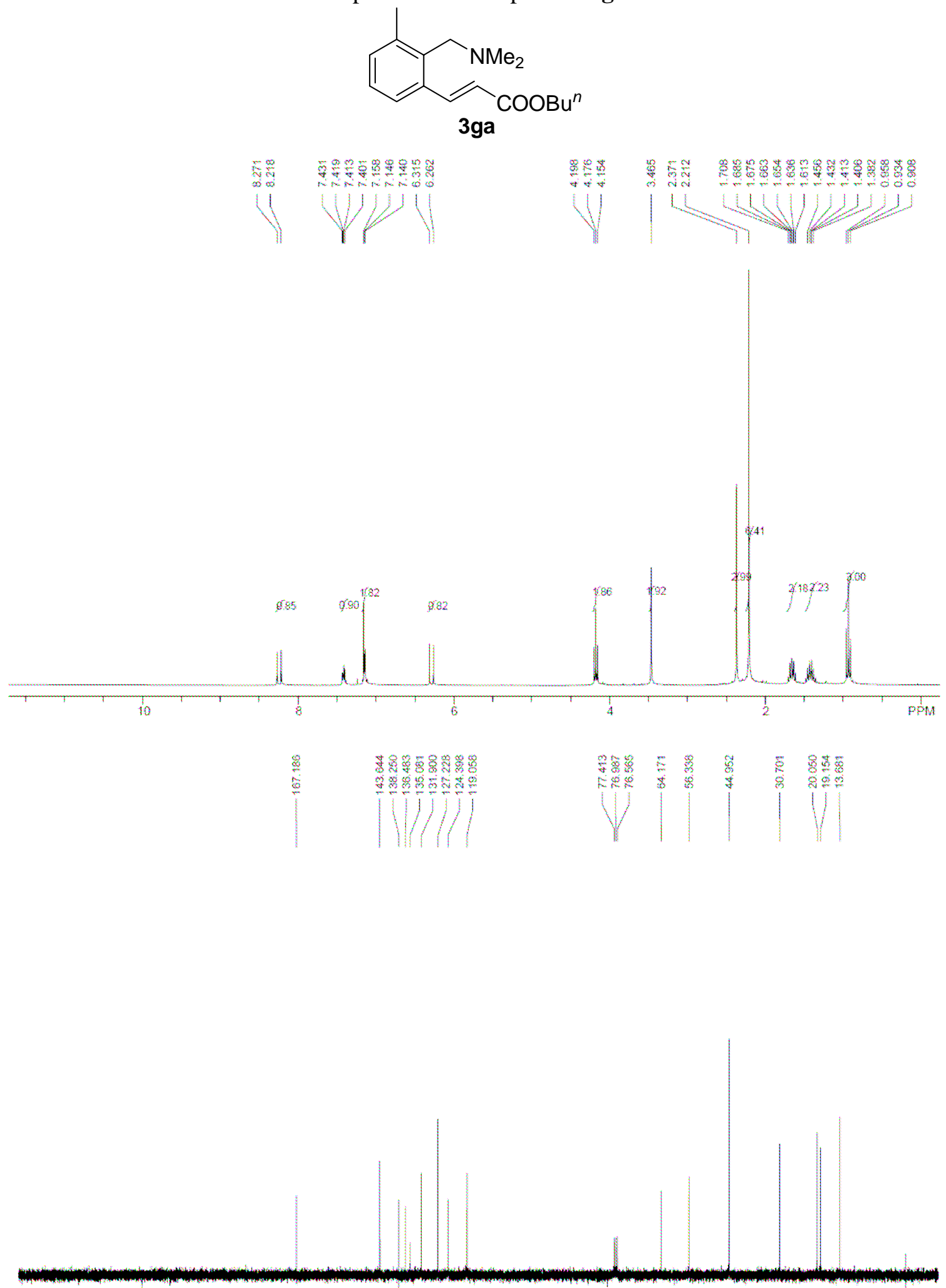


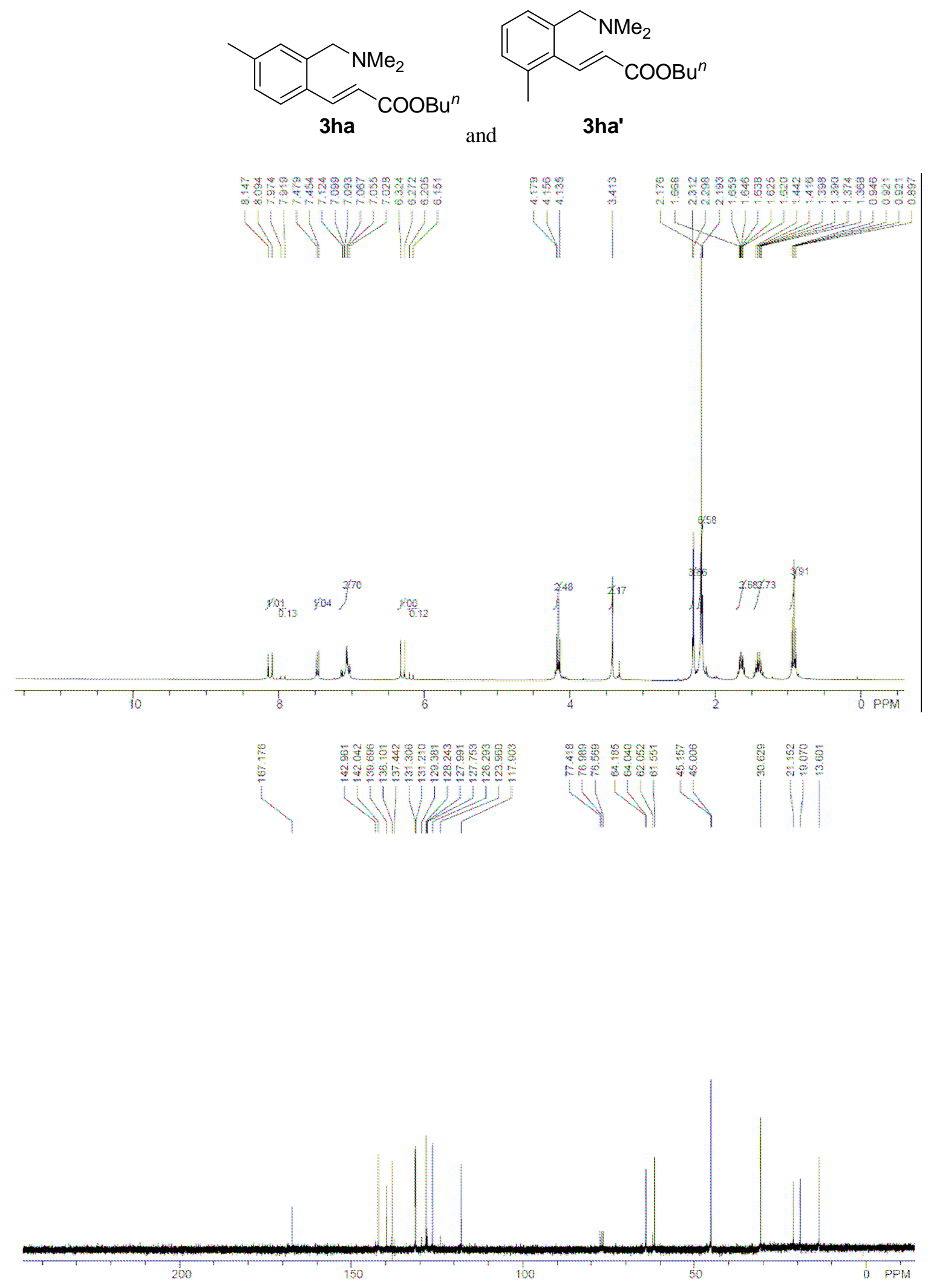



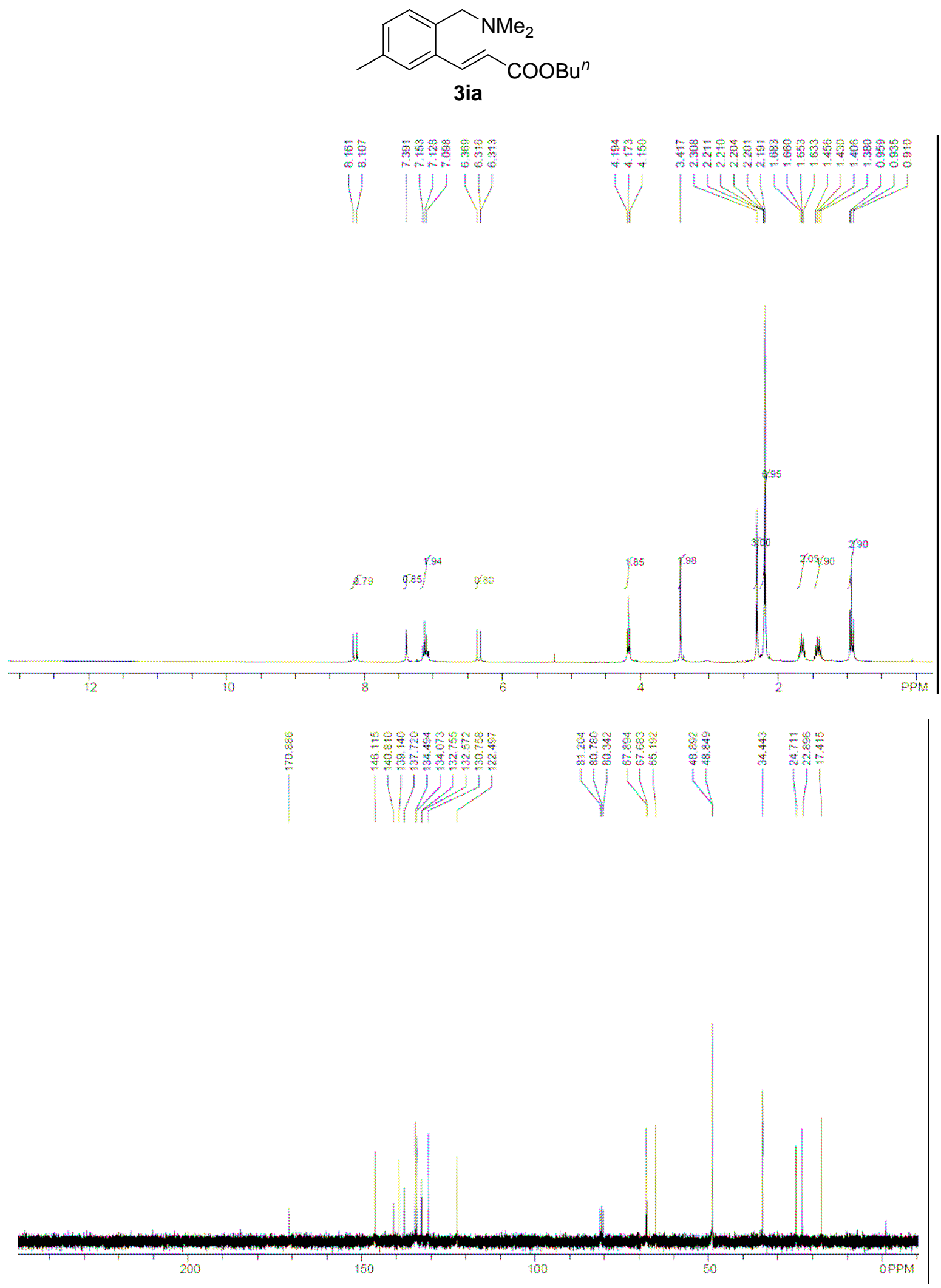

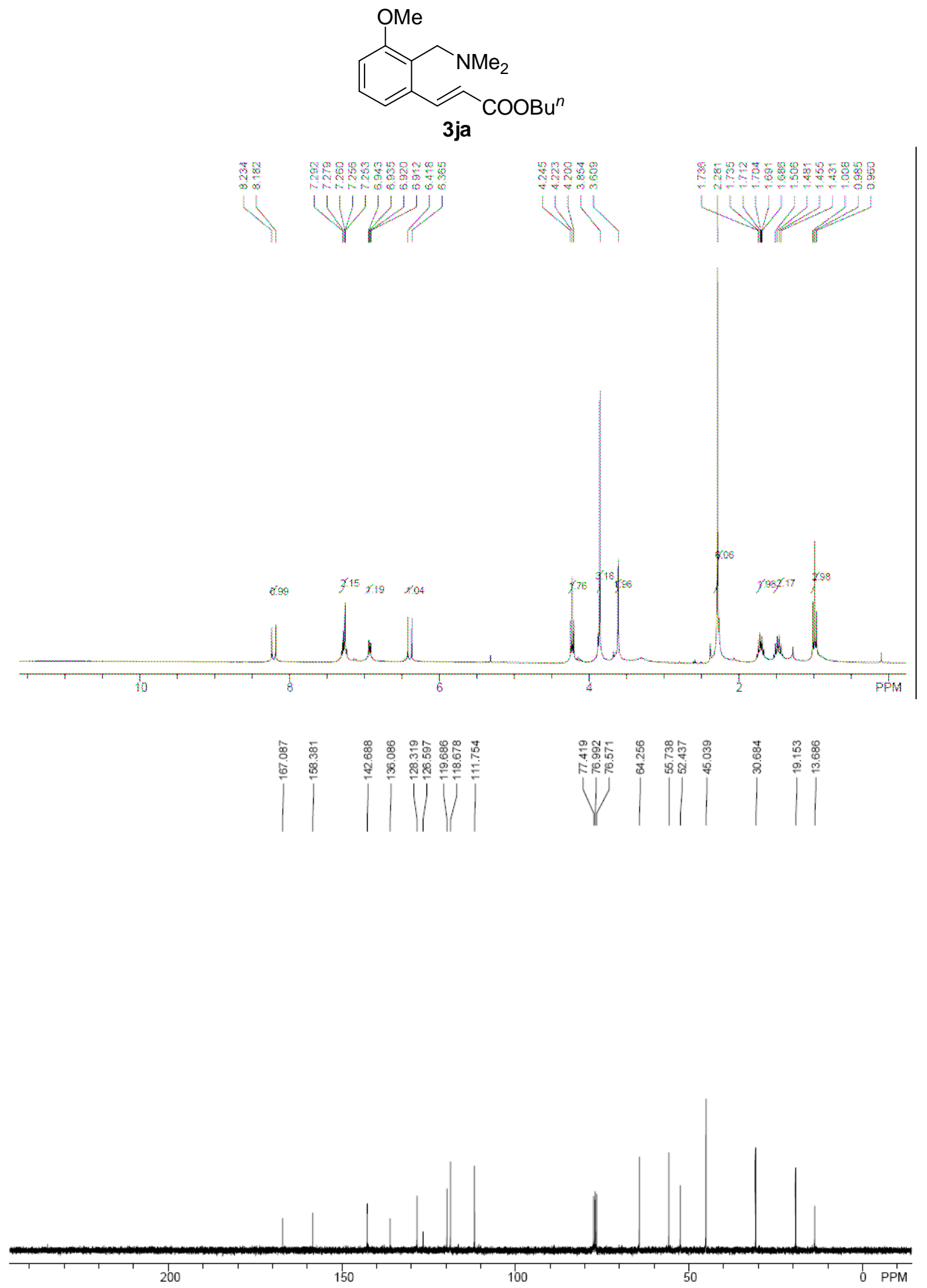


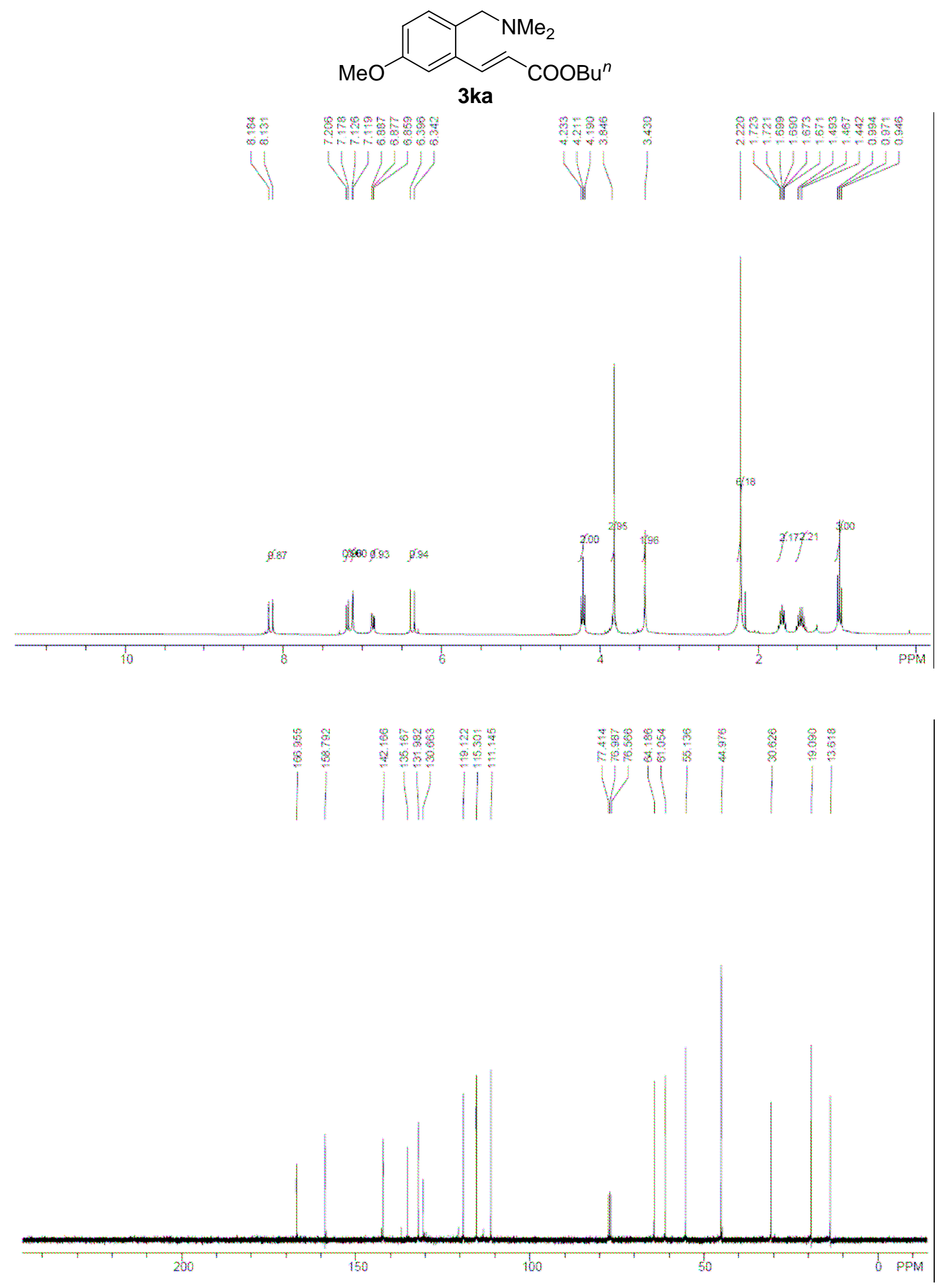




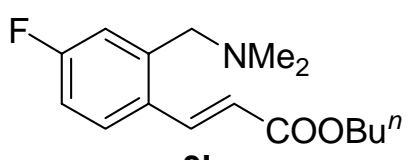

and $\overbrace{3 \mathbf{F}^{\prime}}^{\mathrm{COOBu}_{2}}$
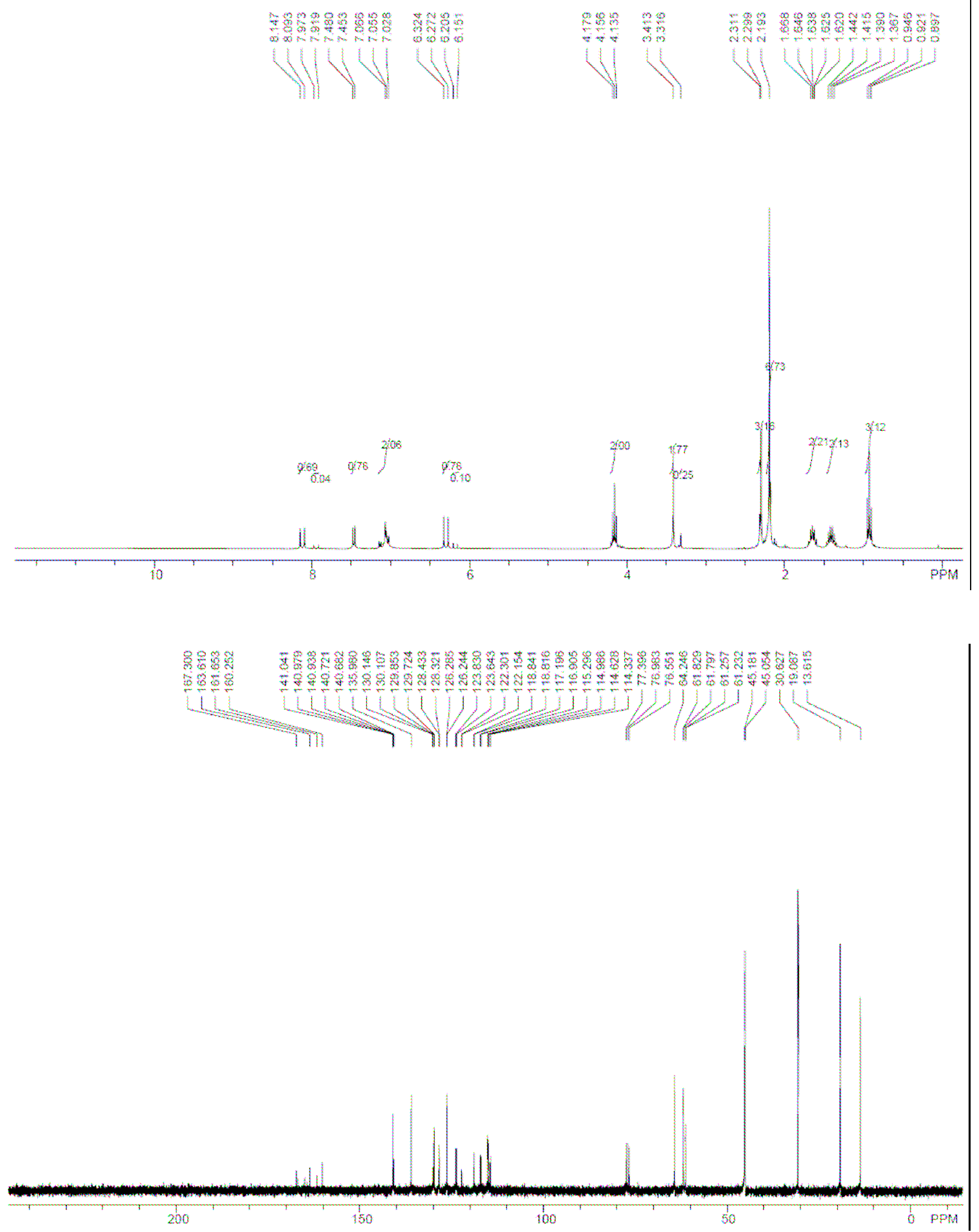

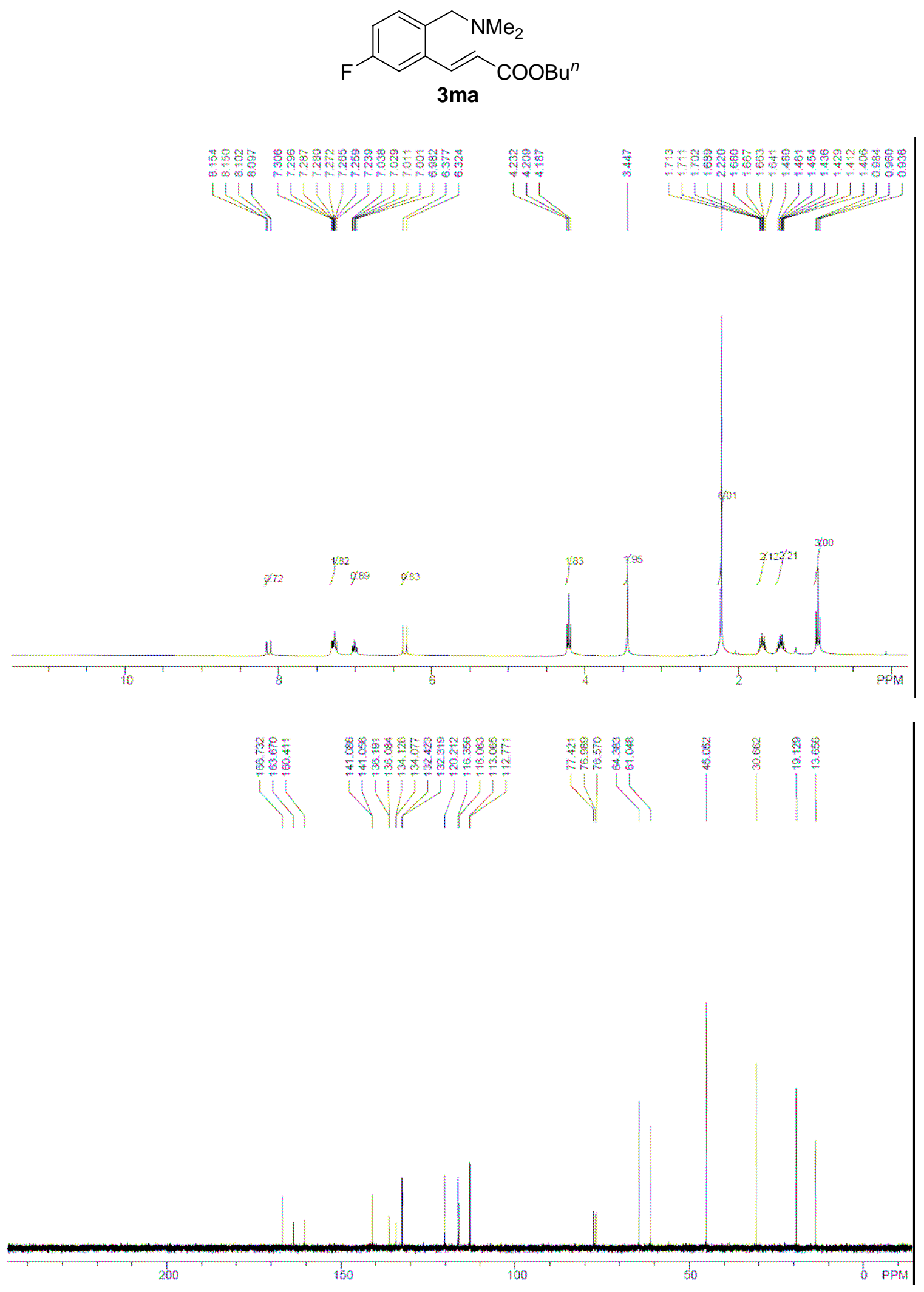

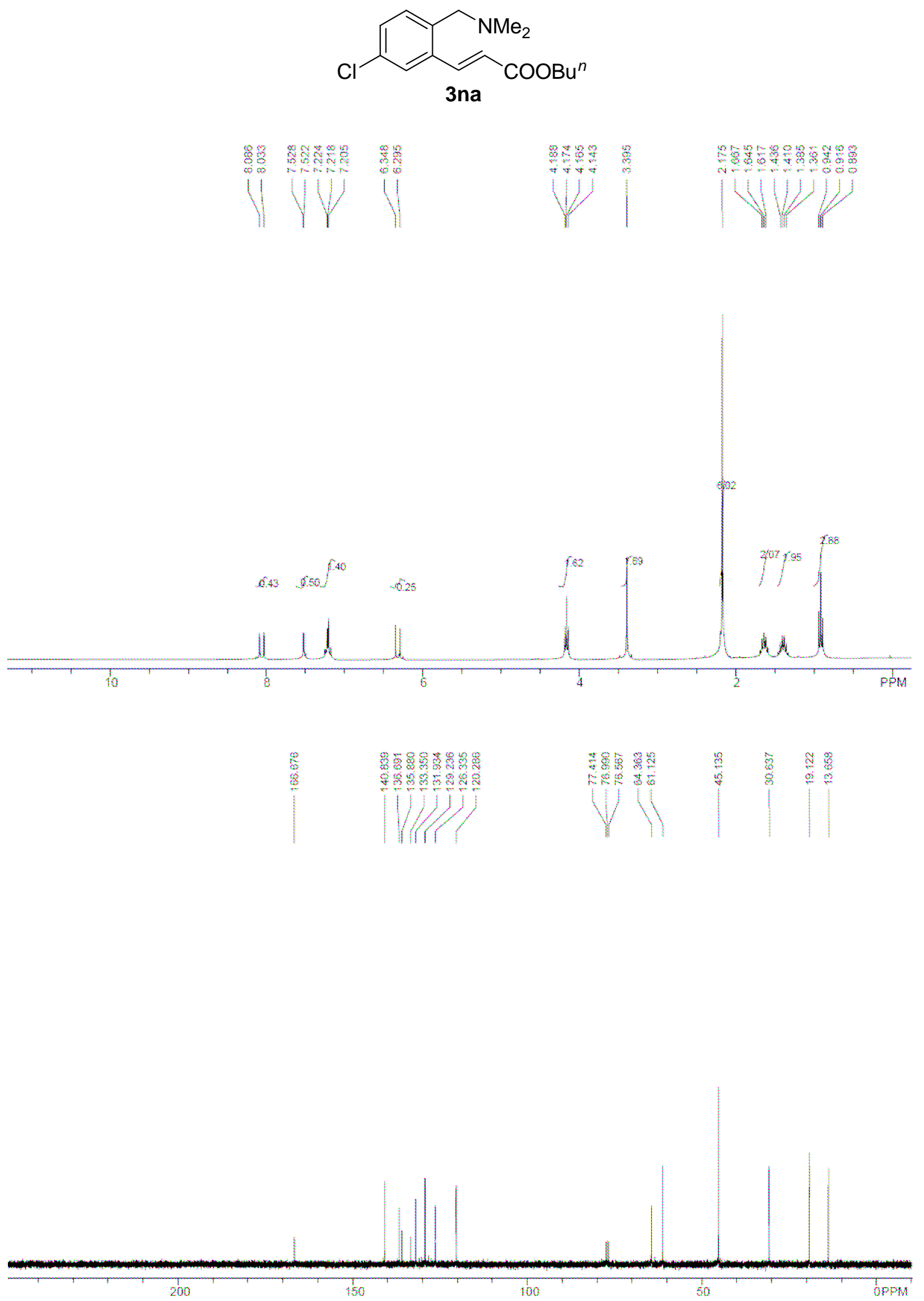

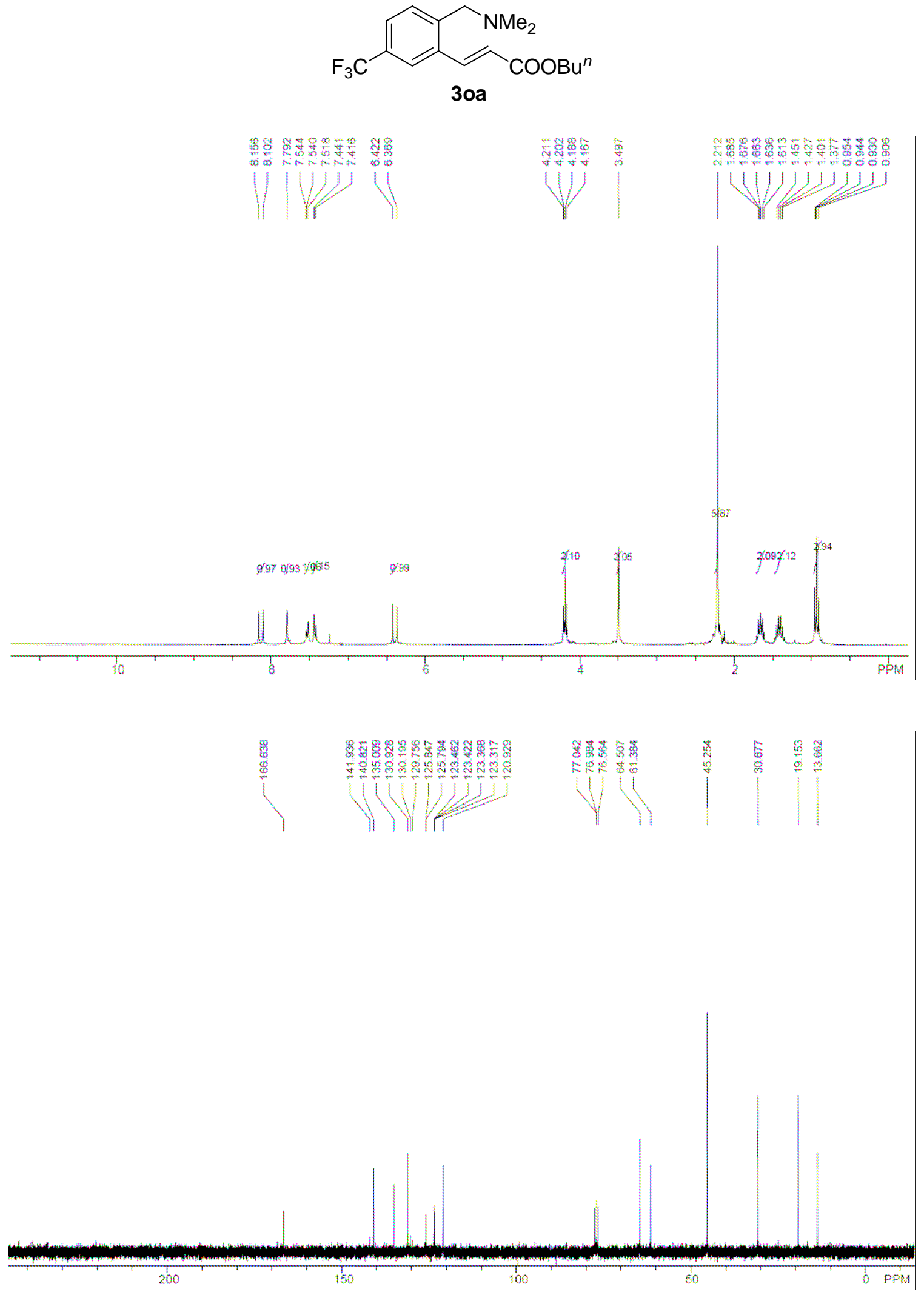

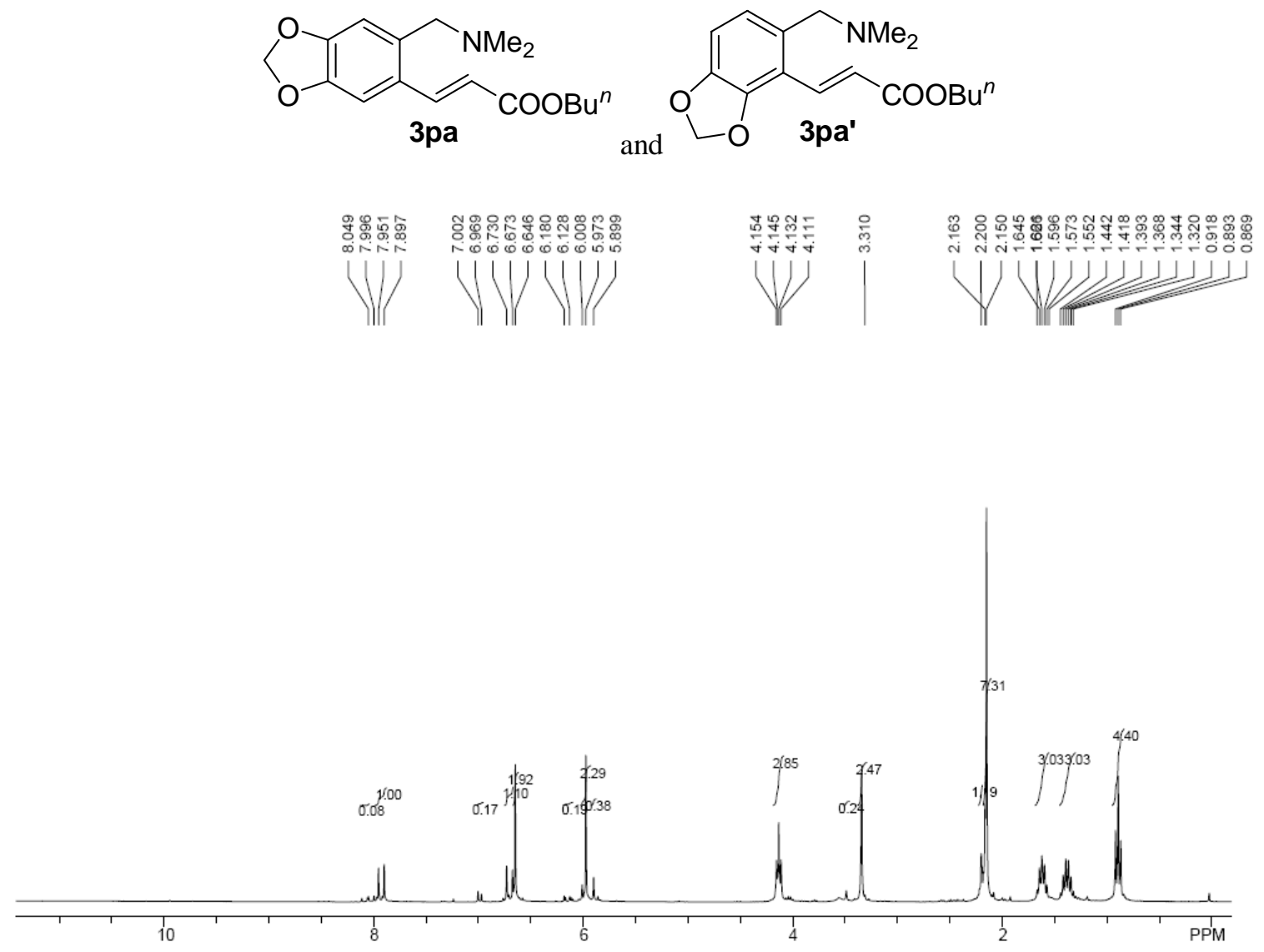

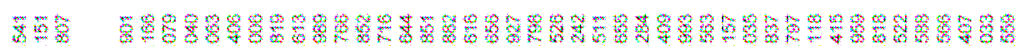

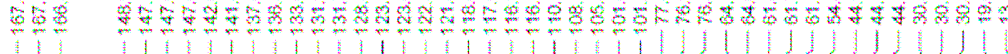

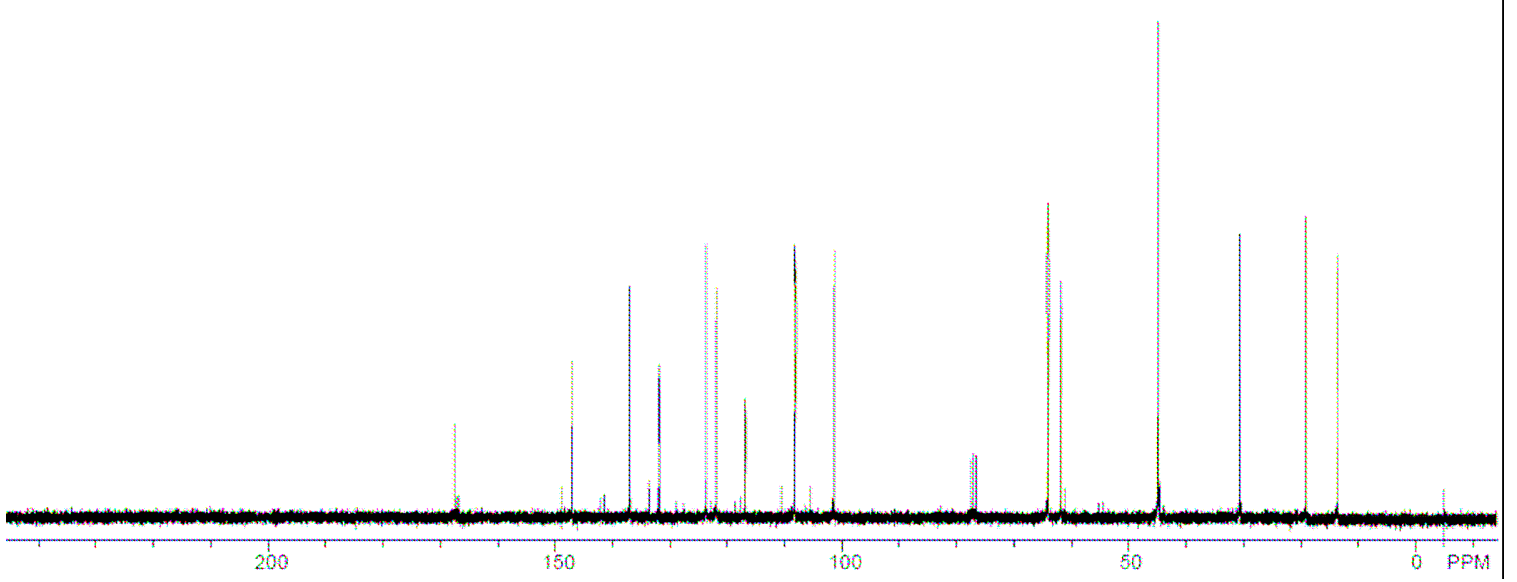



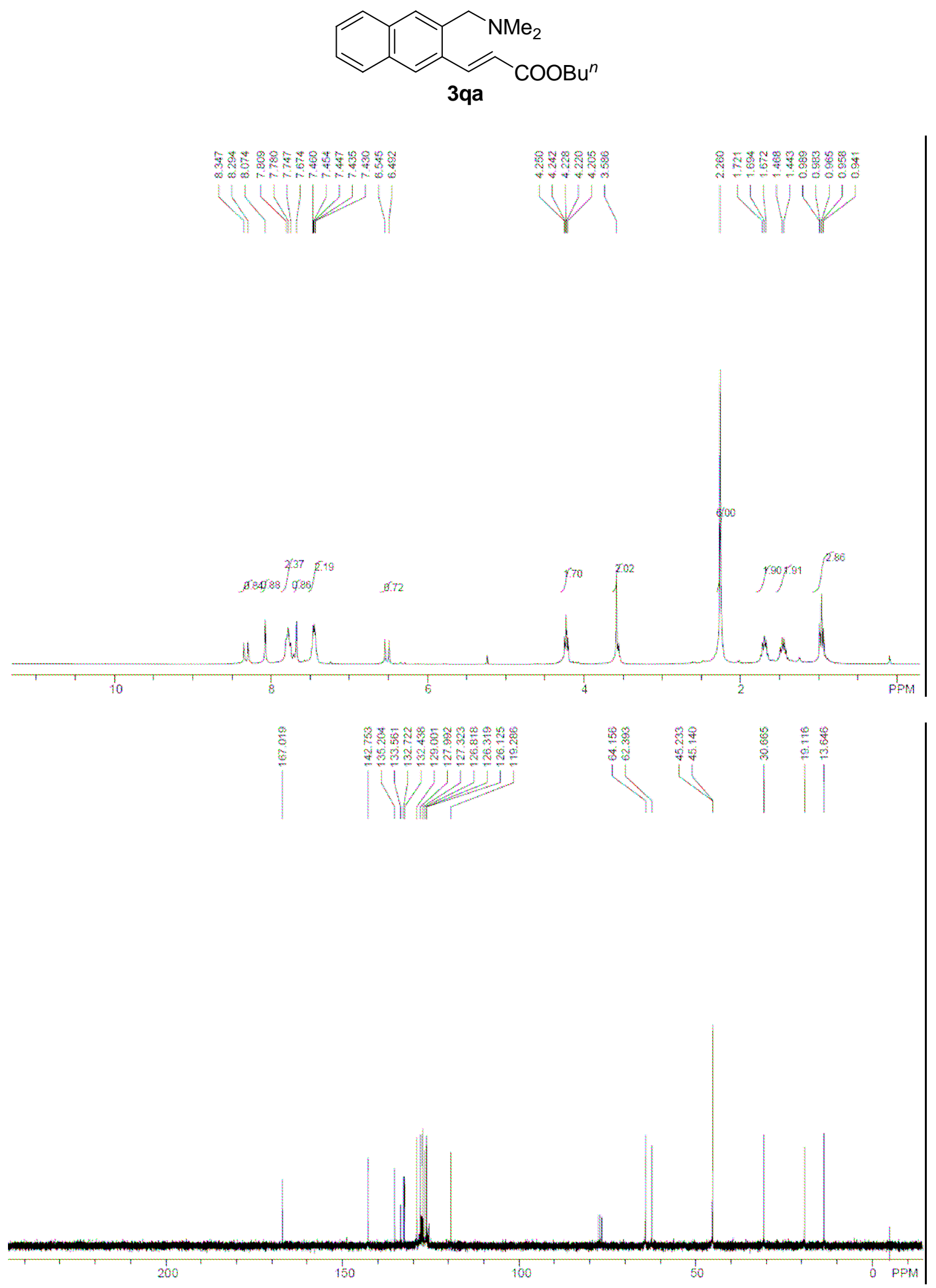

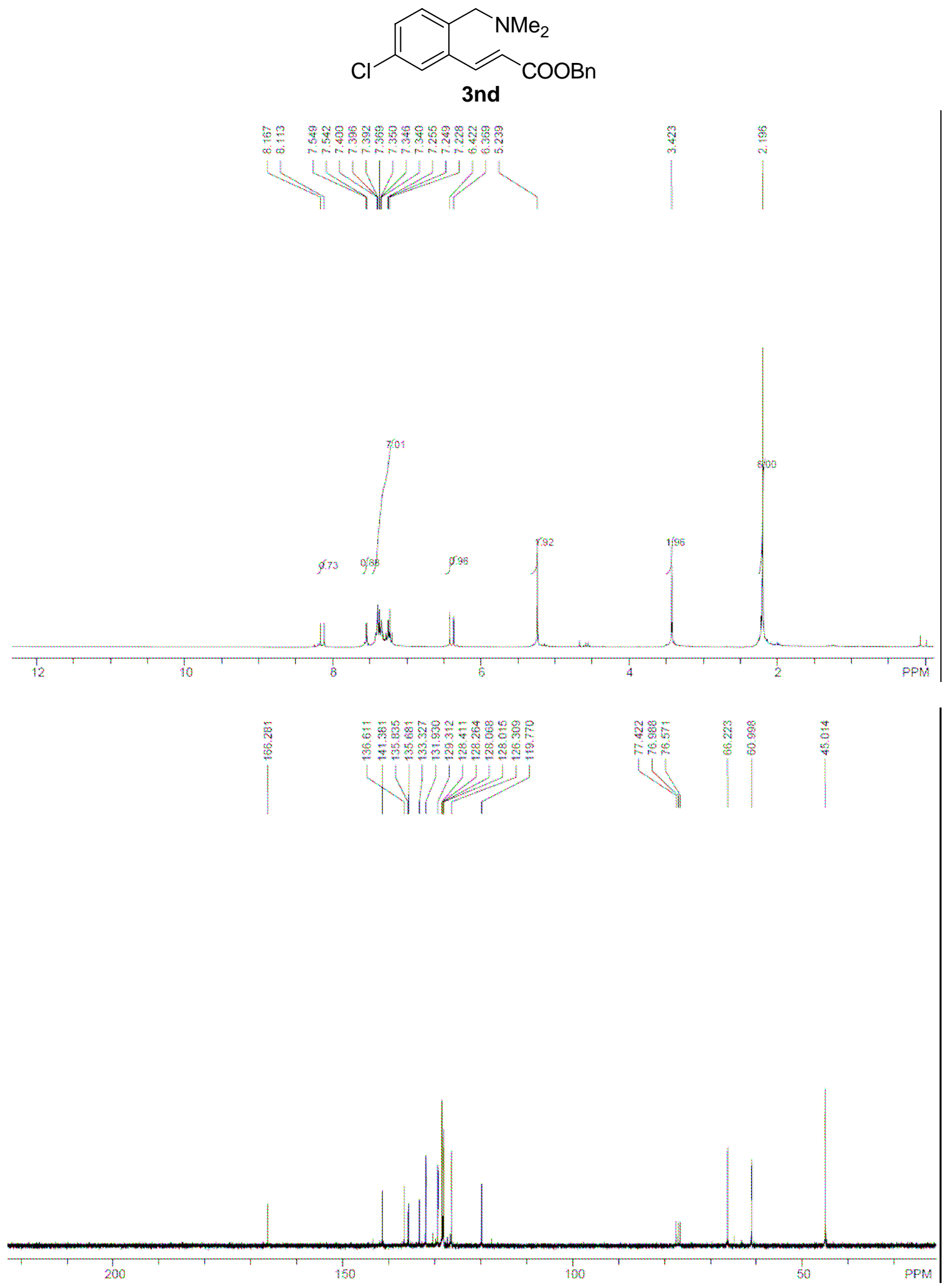

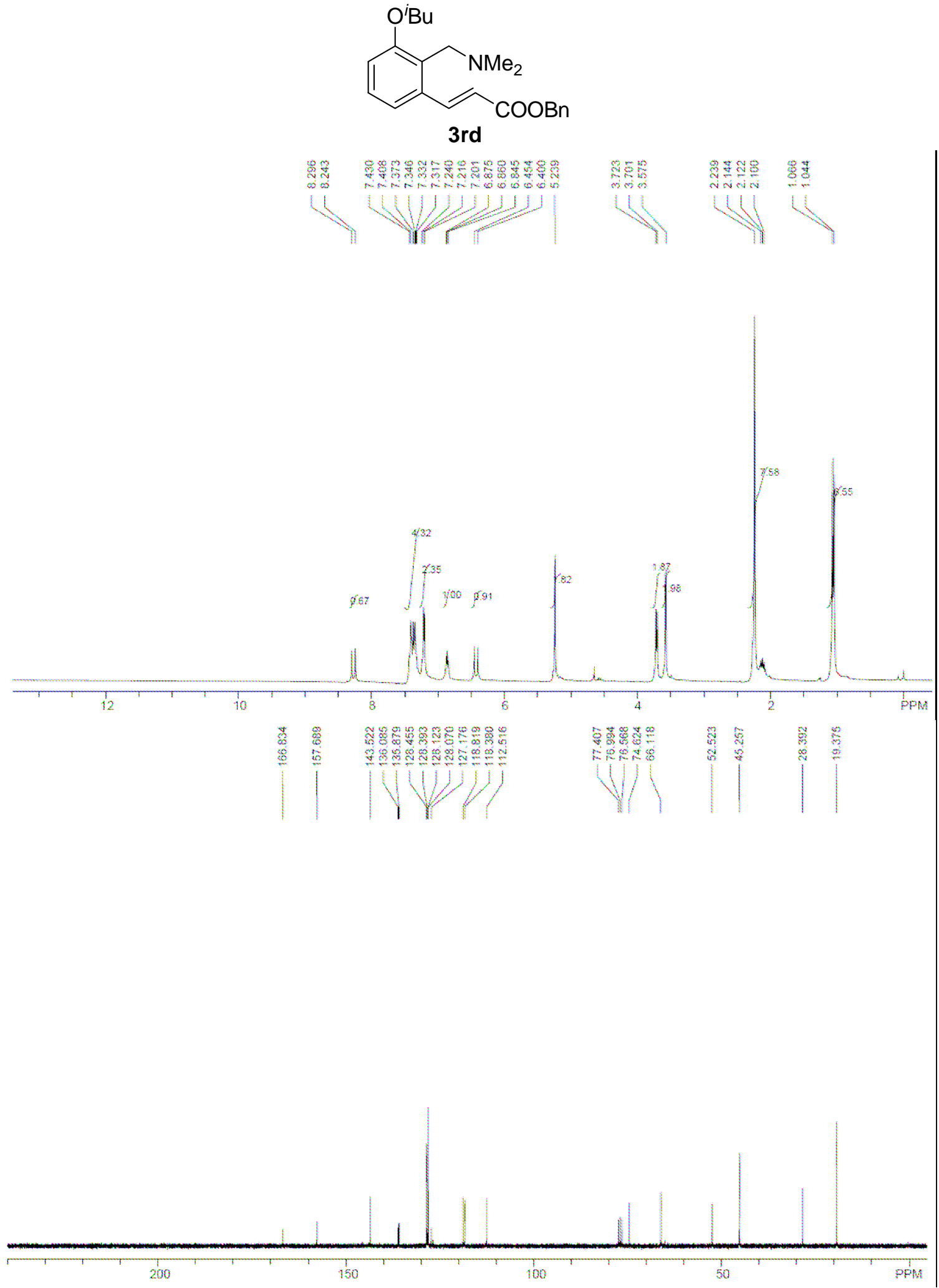
NMR spectrum of compounds 4aa-4rd
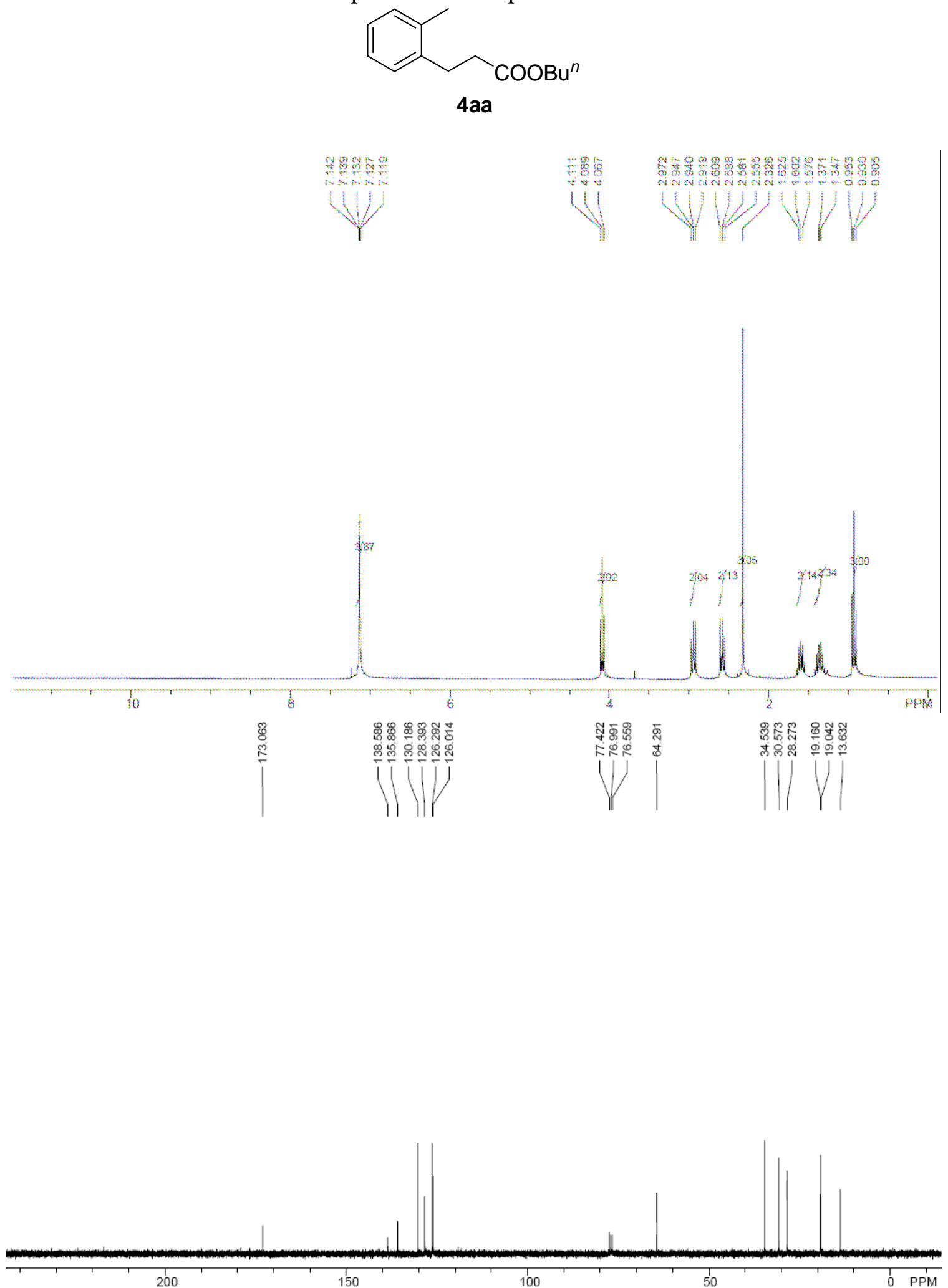

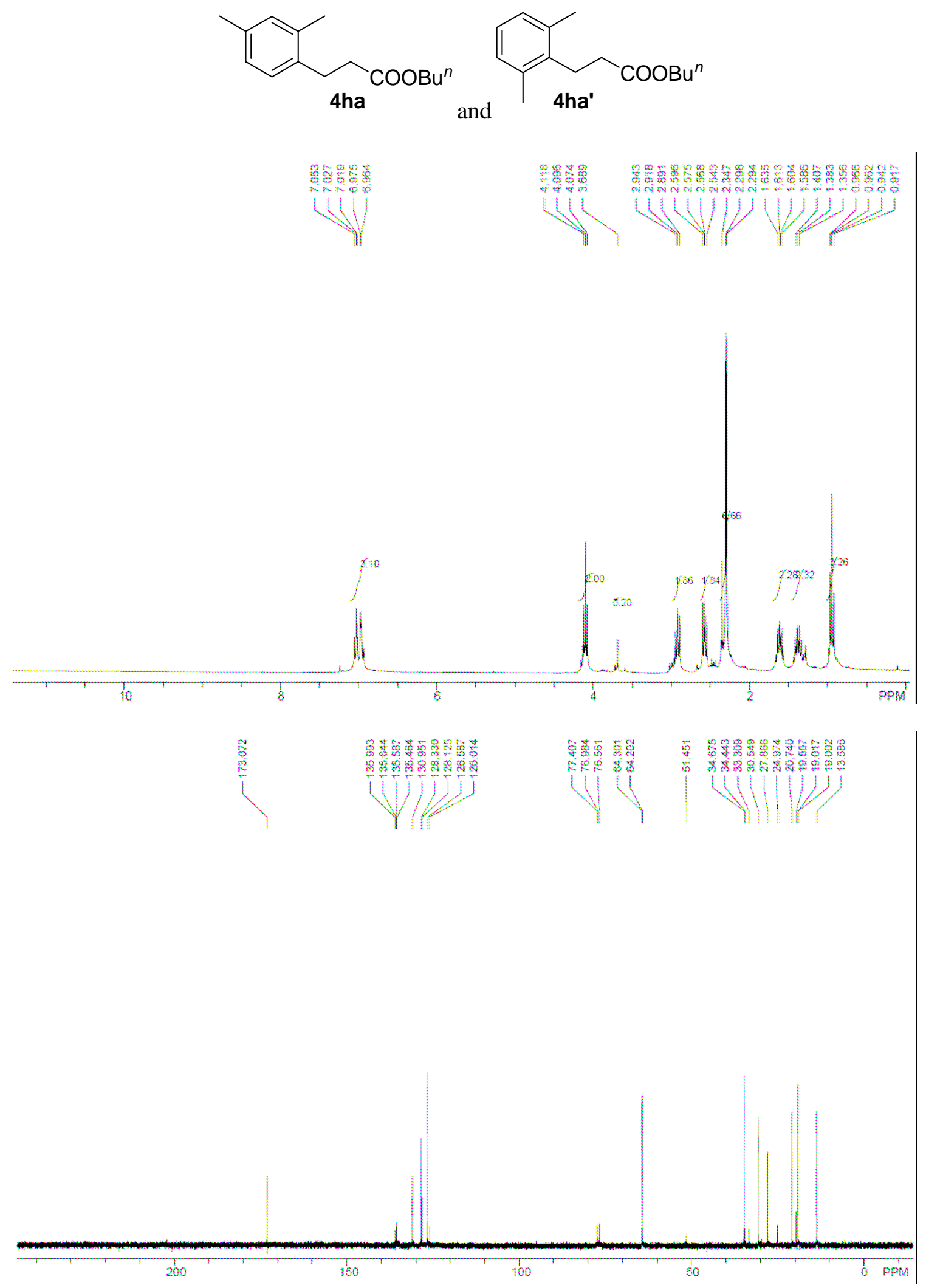


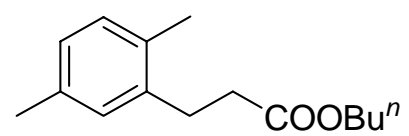

$4 i a$
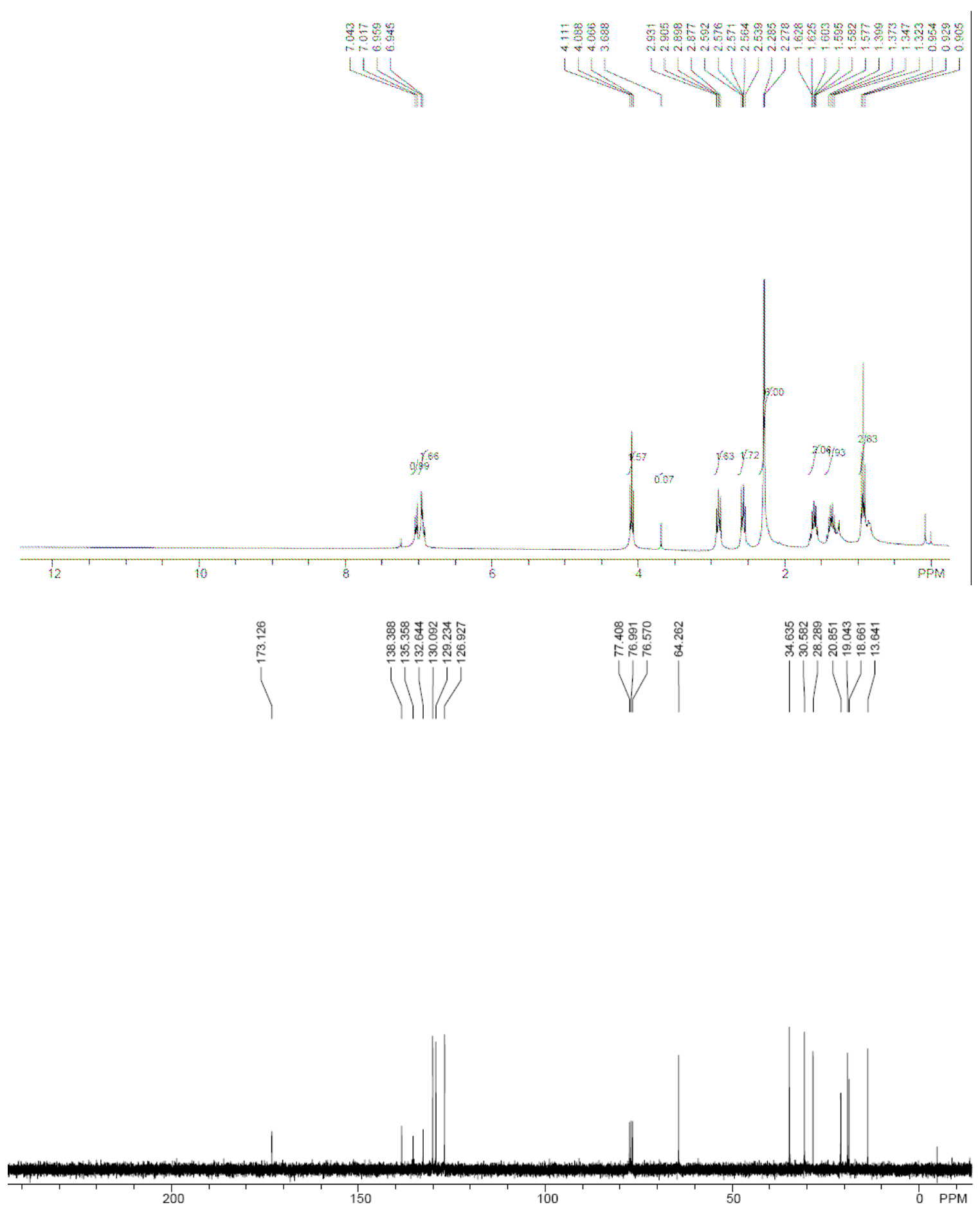


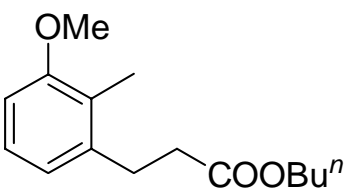

$4 j a$
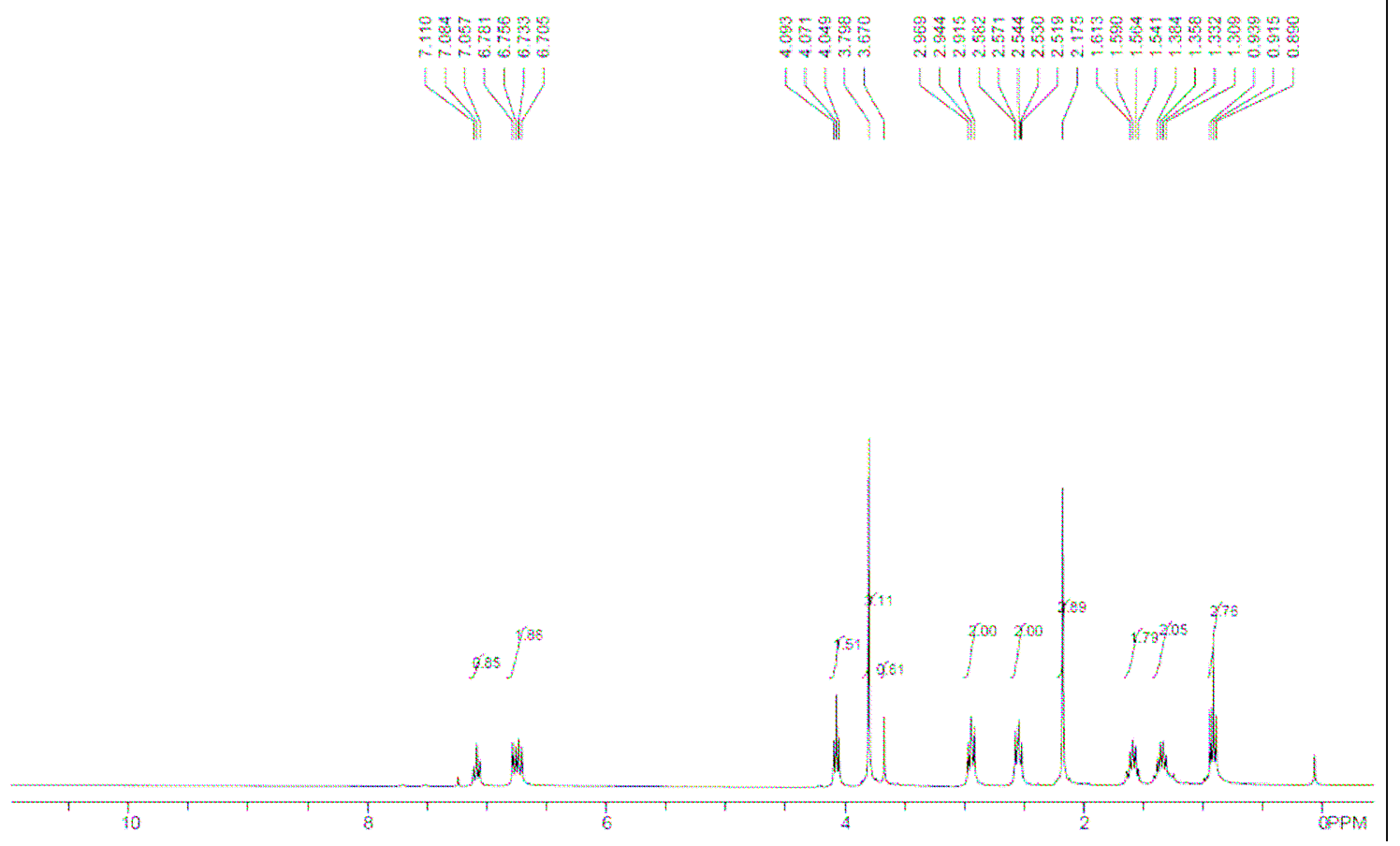

$\left.\right|_{\substack{\infty \\ \infty}} ^{\infty}$

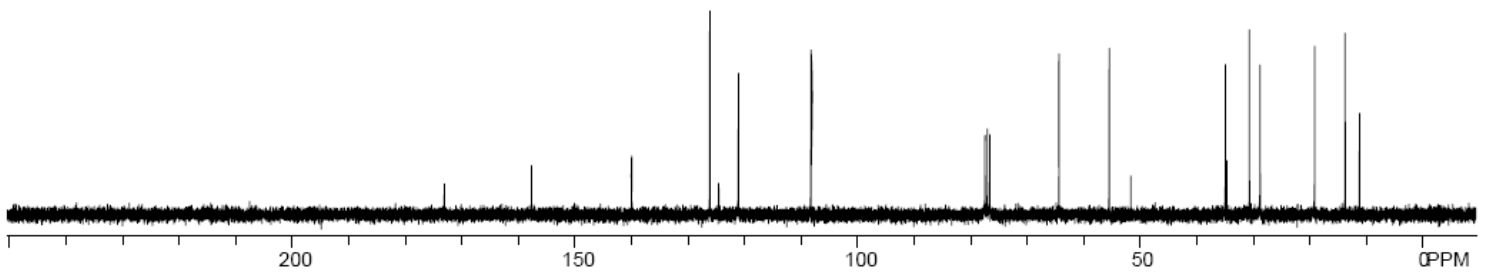




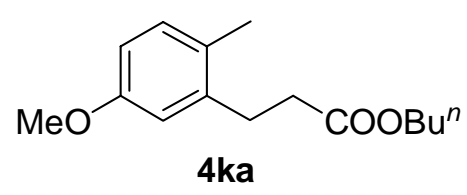

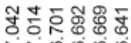

14
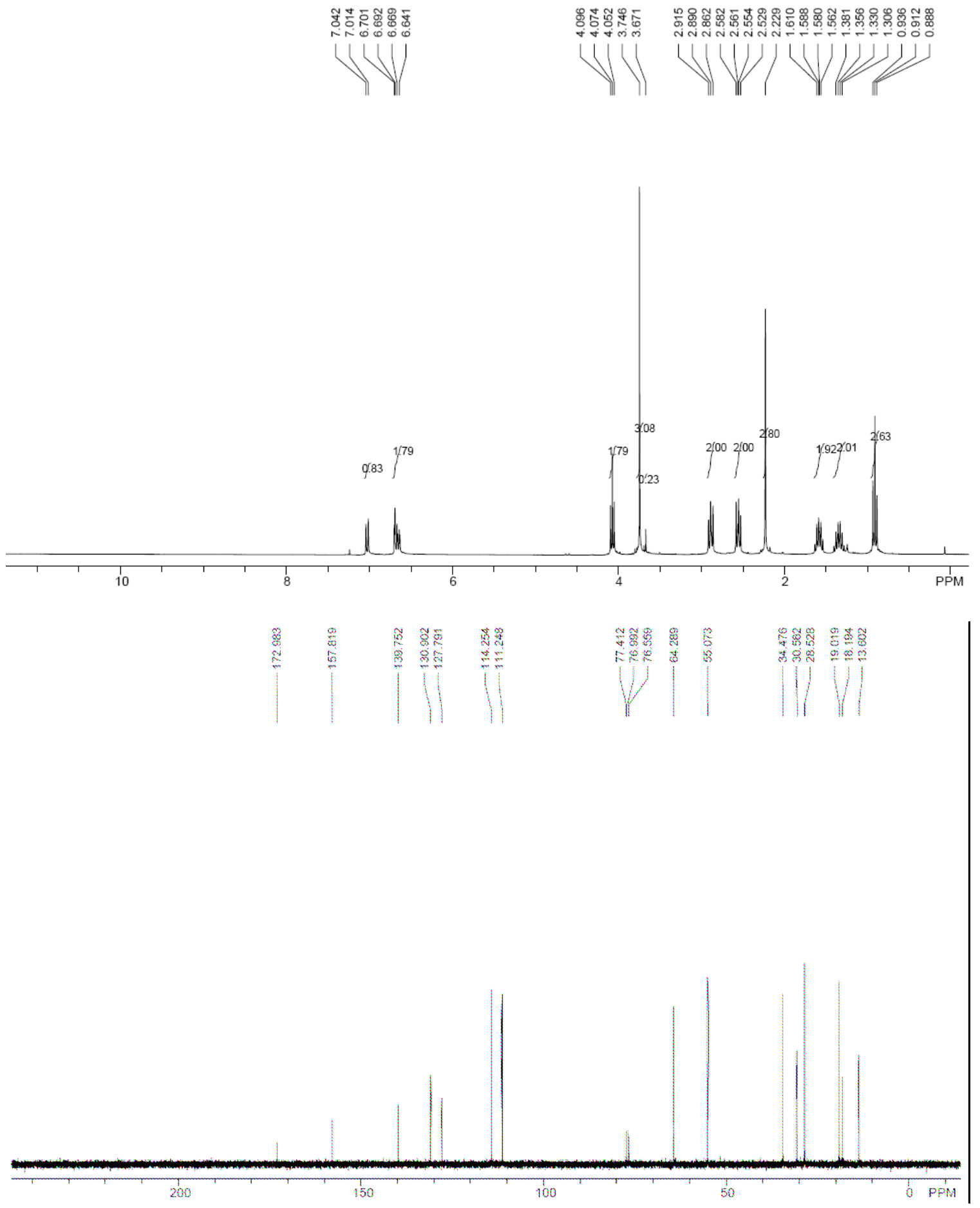


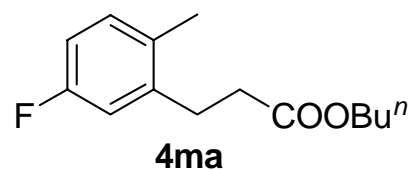

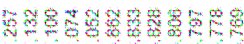
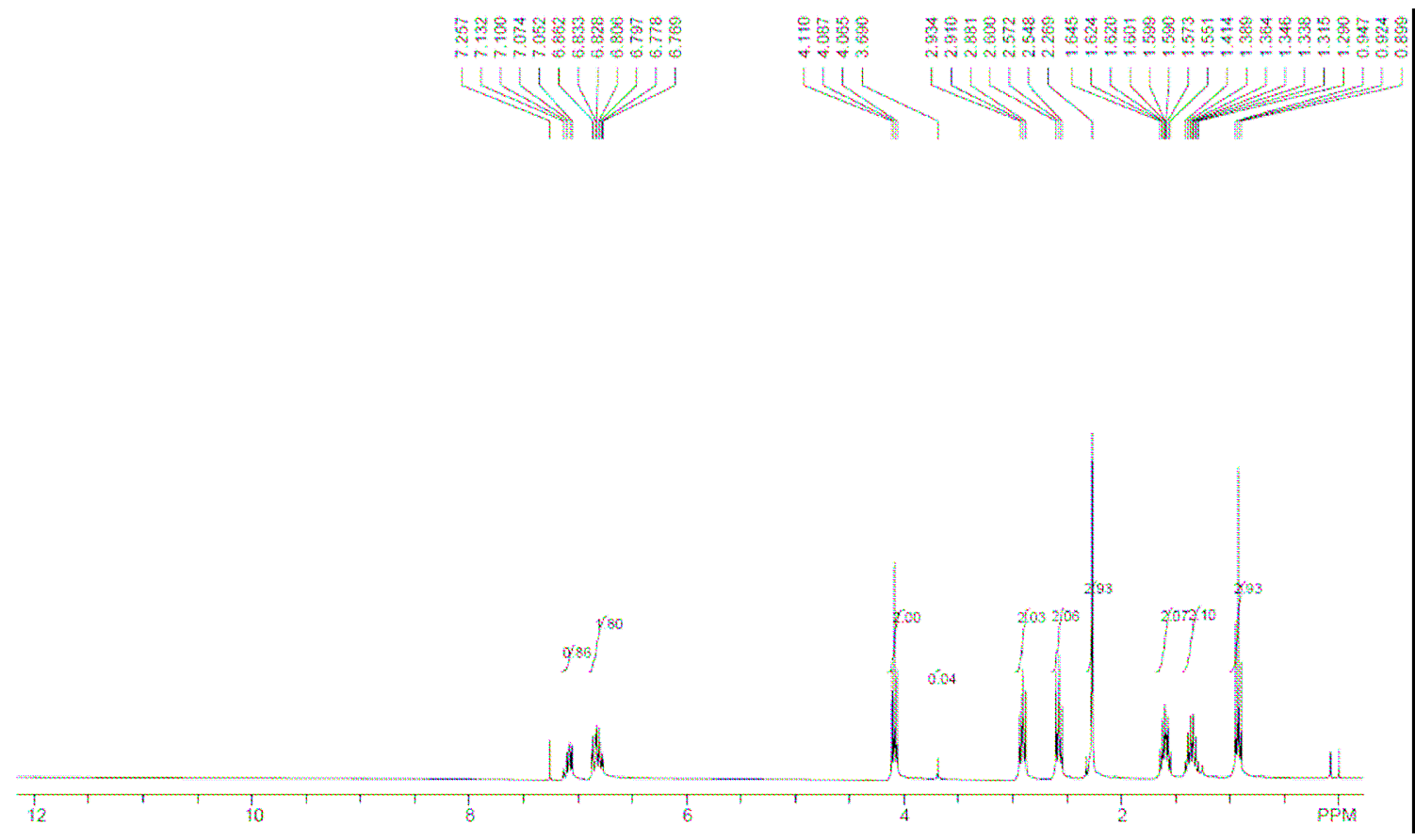

|

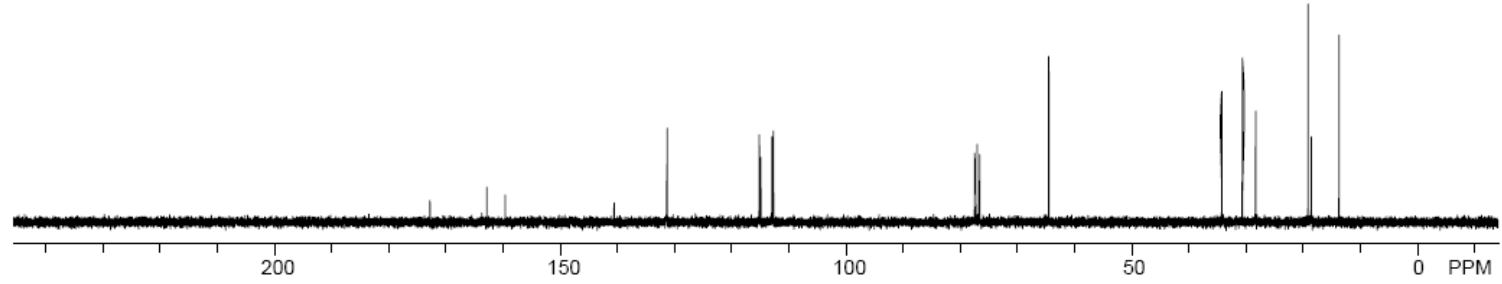




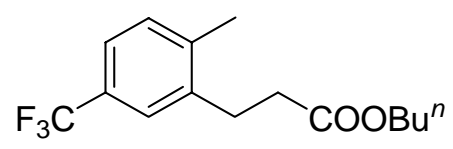

$40 a$
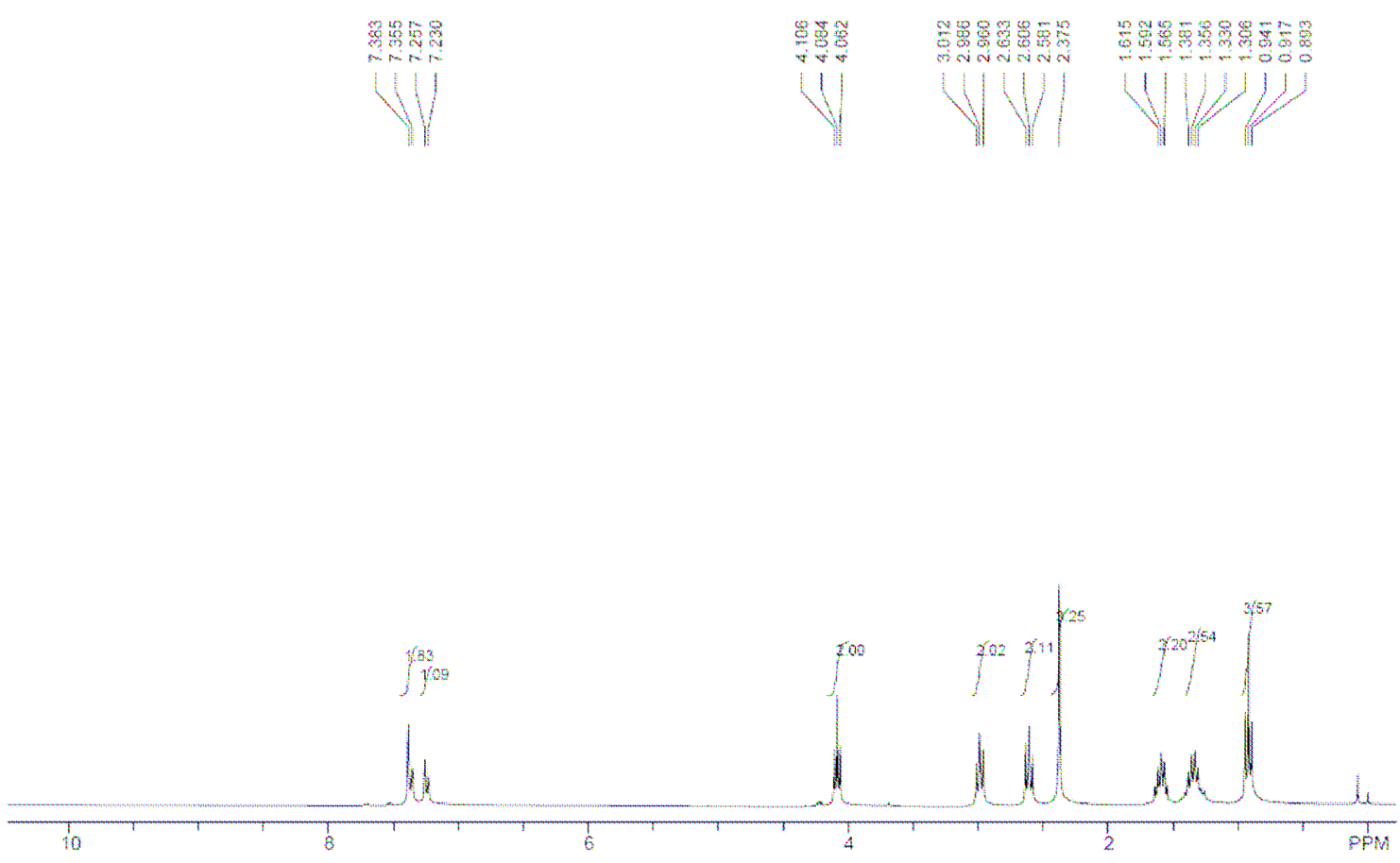

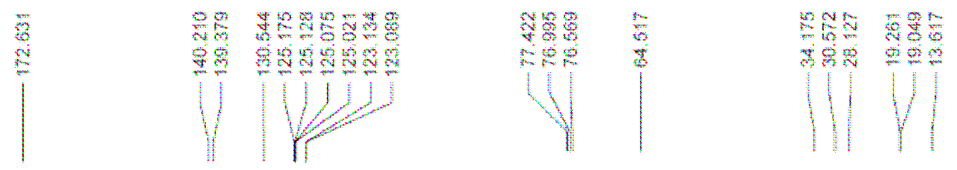

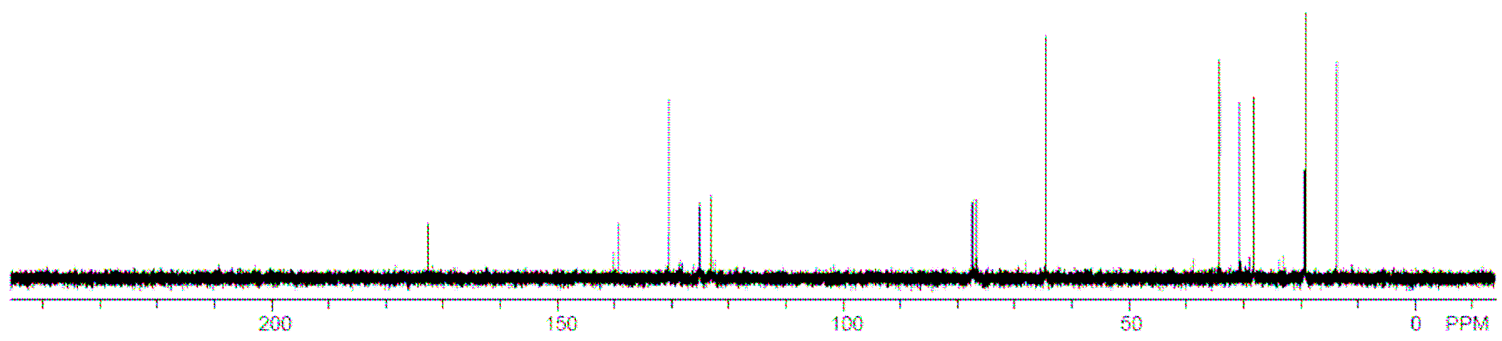



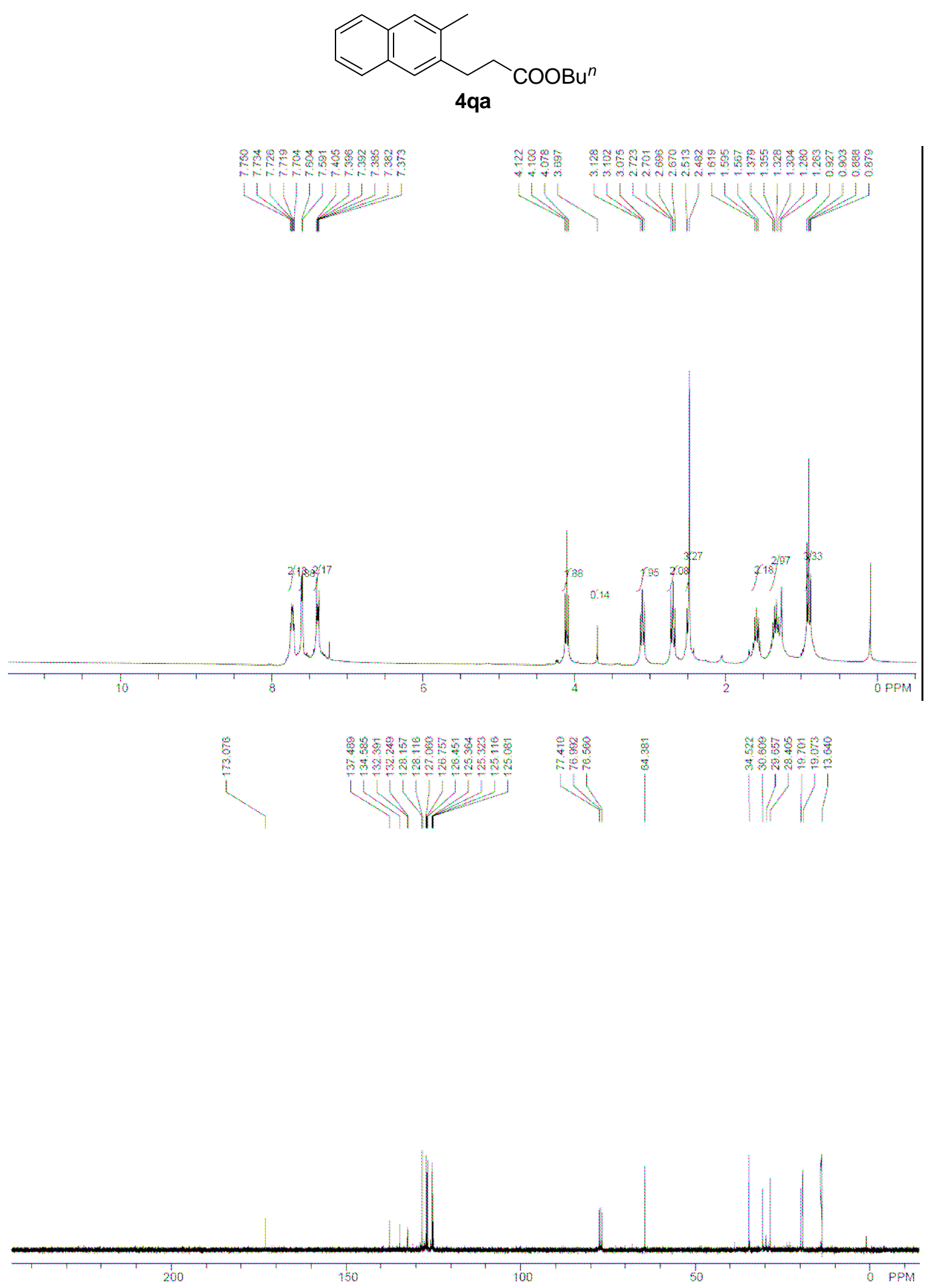


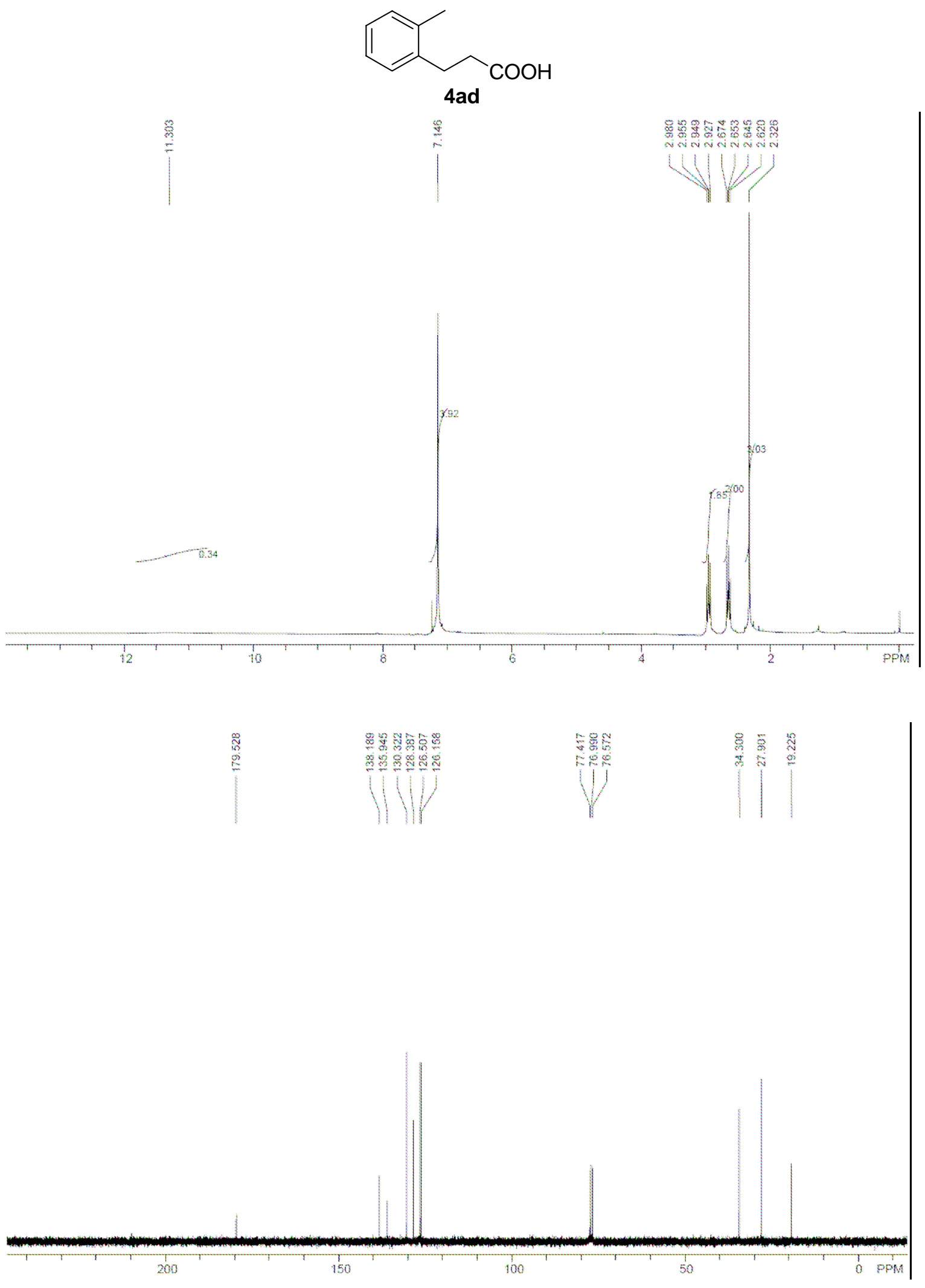



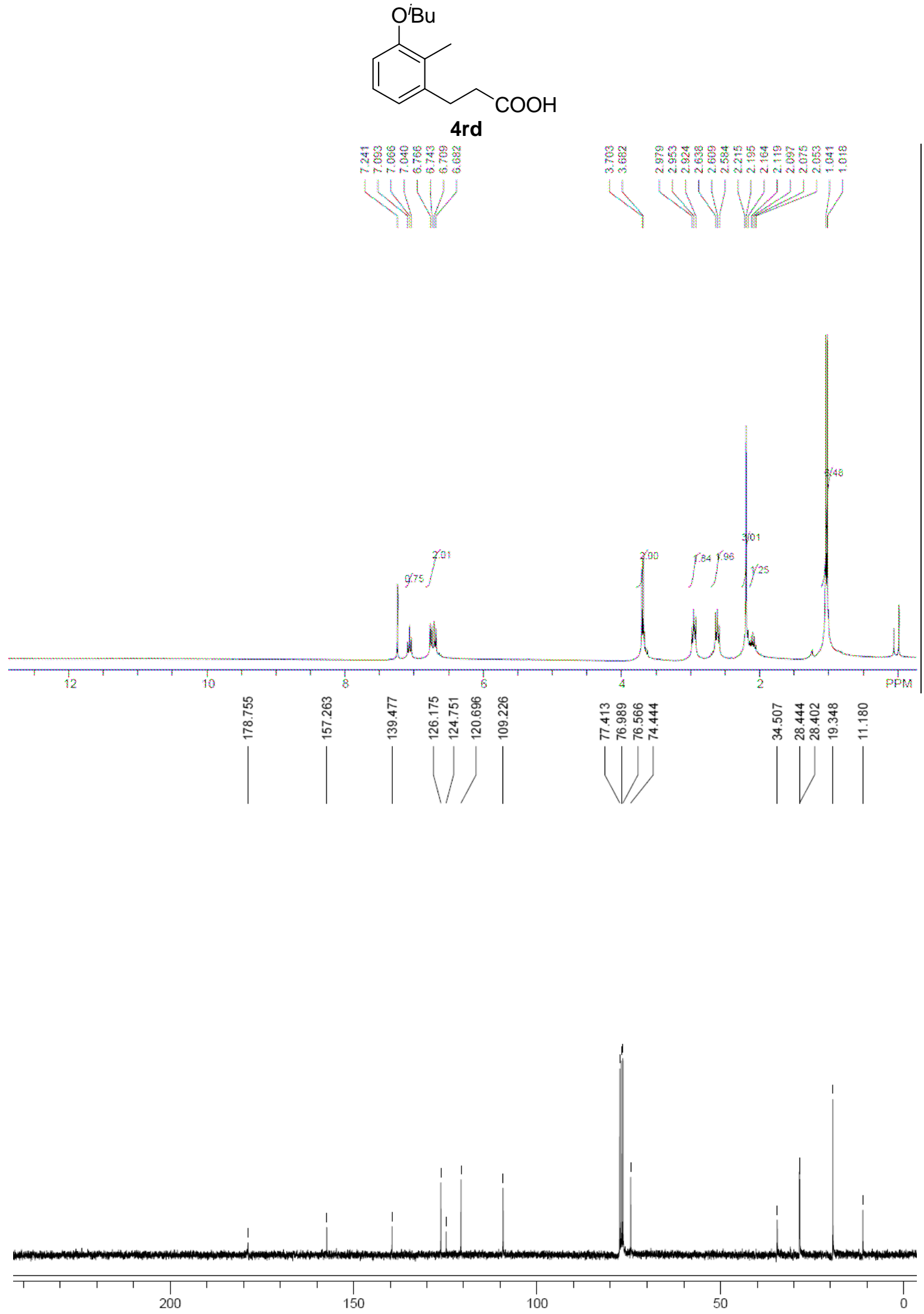


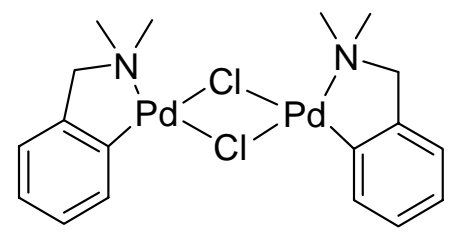

5
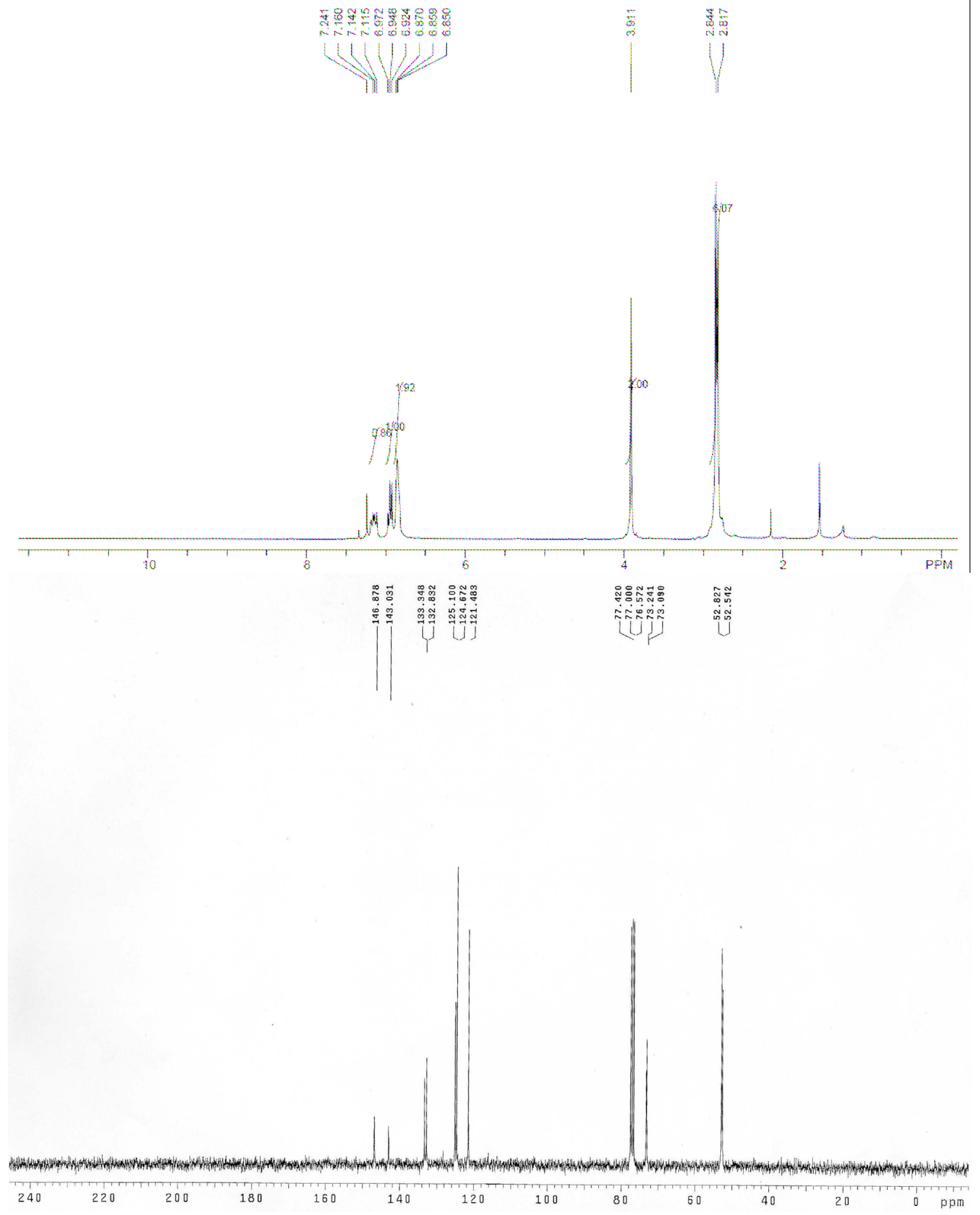


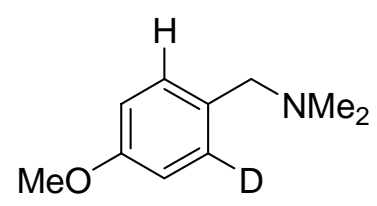

\section{$1 \mathrm{k}-\mathrm{d}$}
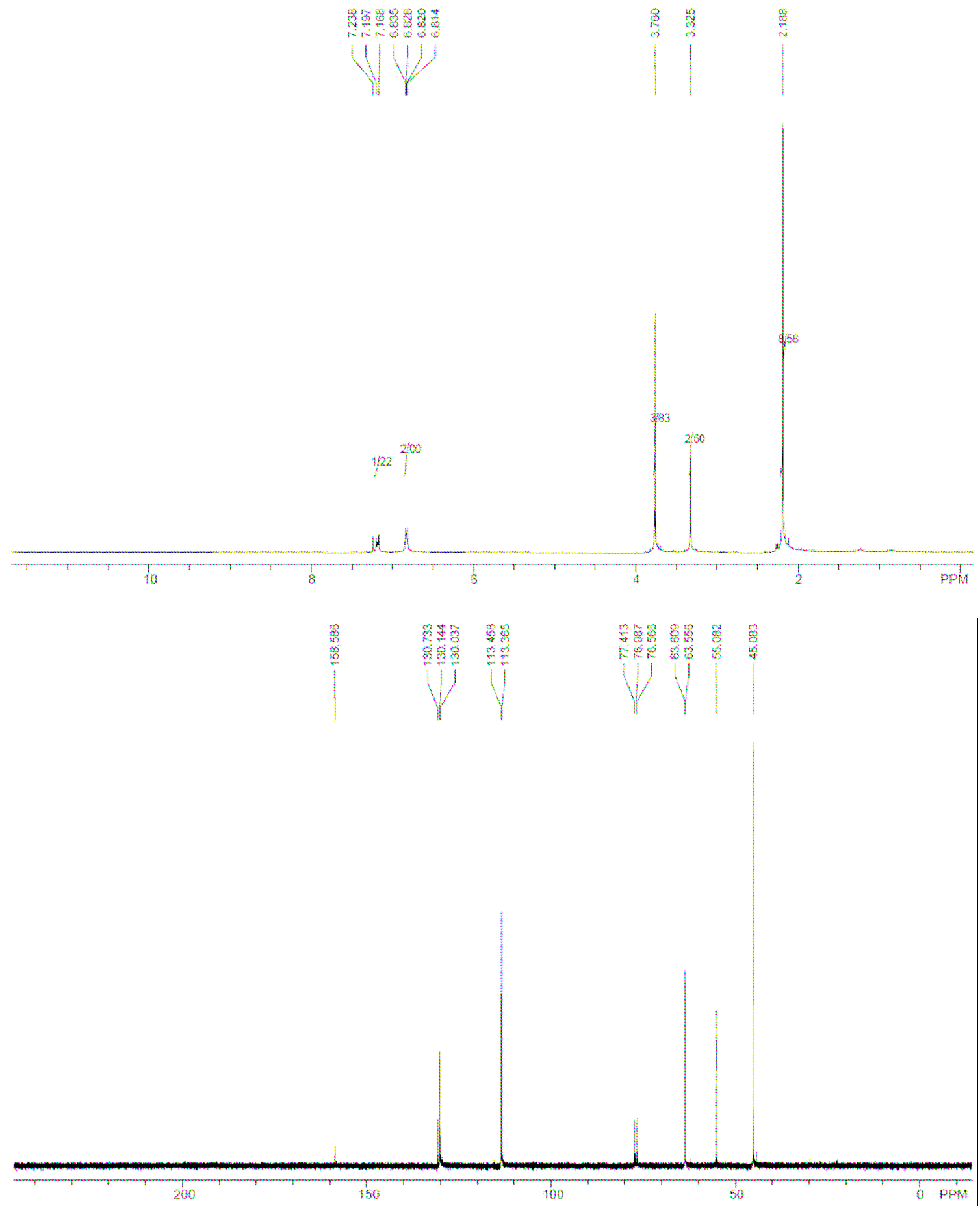
<smiles>[2H]c1cc(OC)cc(/C=C/C(=O)OCC)c1CN(C)C</smiles>

3ka
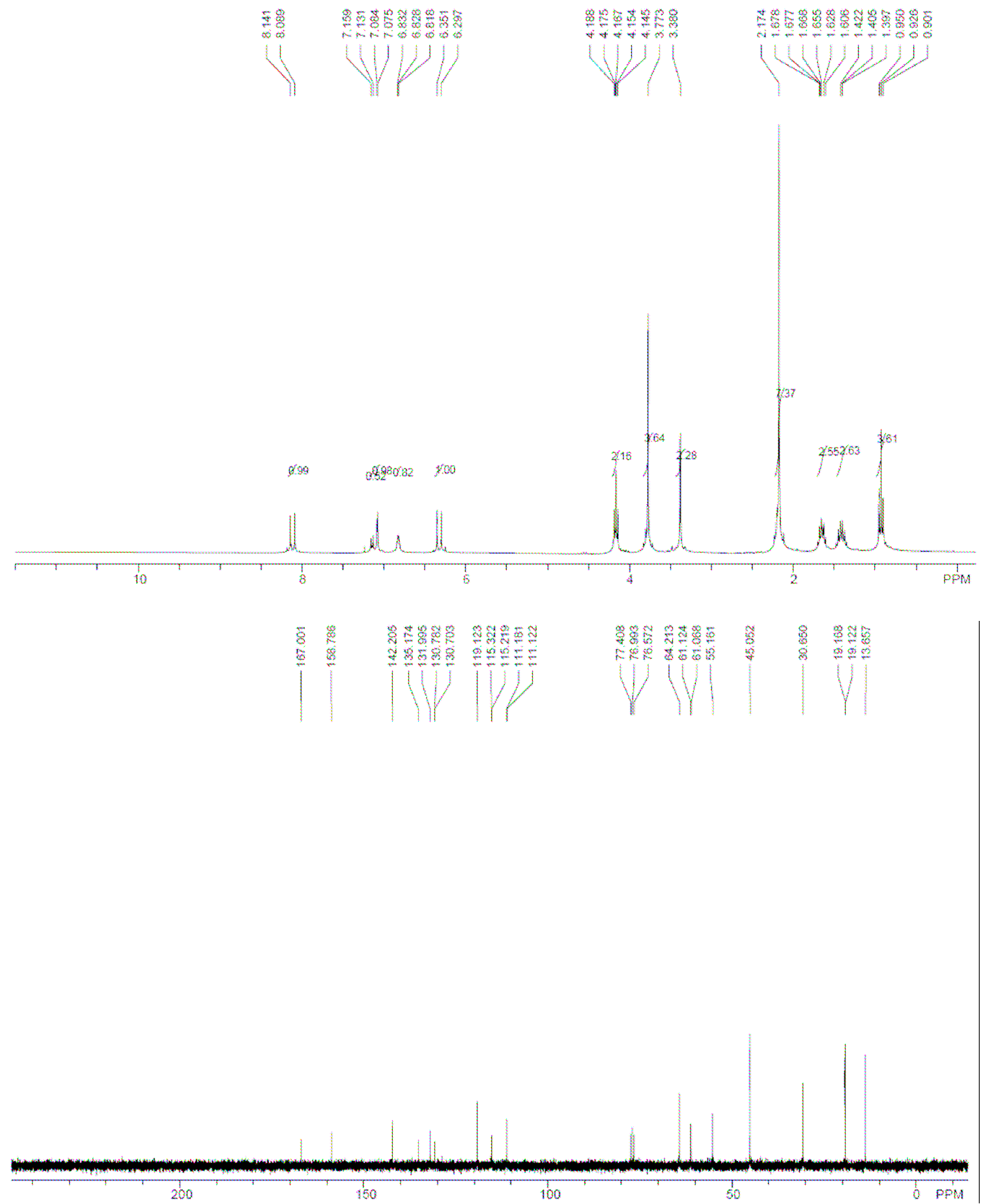